\title{
Latin American Strategy for Research Infrastructures for High Energy, Cosmology, Astroparticle Physics LASF4RI for HECAP
}

\section{LATIN AMERICAN HECAP PHYSICS BRIEFING BOOK}

\author{
Preparatory Group
}

Hiroaki Aihara - University of Tokyo

Reina Camacho Toro- LPNHE/CNRS

Marcela Carena - Fermilab/U. of Chicago

Juan Carlos D'Olivo - UNAM

Thiago Goncalves - Valongo Observatory

Diana López Nacir - DF/IFIBA UBA-CONICET

Jorge Molina - Universidad Nacional de Asunción

Diego Restrepo - Universidad de Antioquia,

Arturo Sánchez- ICTP/INFN/ U. of Udine

Marcelle Soares-Santos - U. Michigan

Hernán Wahlberg - U. Nacional de la Plata

Alfonso Zerwekh - U. Técnica Federico Santa María
Alfredo Aranda - University of Colima

Mauro Cambiaso - Universidad Andrés Bello

Edgar Carrera - Universidad San Francisco de Quito

Alberto Gago - Pontifica Universidad Católica del Perú

Gerardo Herrera - CINVESTAV

Marta Losada - NYUAD

Martijn Mulders - CERN

Rogerio Rosenfeld - IFT-UNESP \& ICTP-SAIFR

Federico Sánchez - U. Nacional de San Martín

Martin Subieta - U. Mayor de San Andrés

Harold Yepes Ramirez - YTU 


\section{Contents}

1 Introduction 1

2 Astronomy, Astrophysics and Astroparticle Physics 4

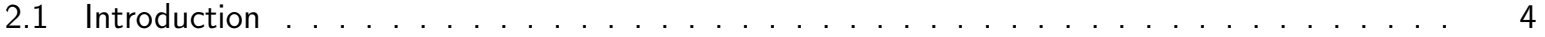

2.2 Involvement of Latin American Countries . . . . . . . . . . . . . . . . . . . . G

2.2 .1 Pierre Auger Observatory . . . . . . . . . . . . . . . . . . . . 6

2.2.2 Latin American Giant Observatory . . . . . . . . . . . . . . . . . . . . . . . 7

2.2.3 Cherenkov Telescope Array . . . . . . . . . . . . . . . . . . . . . . . 7 7

2.2.4 Southern Wide-field-of-view Gamma-Ray Observatory . . . . . . . . . . . . . . . . . . 7

2.2.5 Large Latin American Millimeter Array . . . . . . . . . . . . . . . . . . . . . . . . 8

2.2.6 Giant Radio Array for Neutrino Detection . . . . . . . . . . . . . . . . . . . . . 8

2.3 Leadership Areas . . . . . . . . . . . . . . . . . . . . . . . . 8

2.4 Drivers for Multiple Approaches . . . . . . . . . . . . . . . . . . . . . . . . . . . . 10

2.5 Synergies . . . . . . . . . . . . . . . . . . . . . . . . . . . . . 10

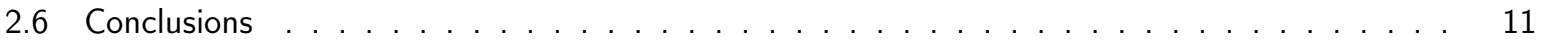

3 Cosmology 14

3.1 Introduction . . . . . . . . . . . . . . . . . . . . . . . . 14

3.2 Experiments and infrastructure with cosmological impact with LA participation . . . . . . 16

3.2.1 BAO from Integrated Neutral Gas Observations (BINGO) . . . . . . . . . . . . . 17

3.2.2 Macon Ridge Astronomical Site: The ABRAS and TOROS projects . . . . . . . . . . 17

3.2.3 Q\&U Bolometric Interferometer for Cosmology (QUBIC) . . . . . . . . . . . . . . . . . 19

3.2.4 South American Gravitational-Wave Observatory (SAGO) . . . . . . . . . . . . . . 20

3.2.5 Vera Rubin Observatory's Legacy Survey of Space and Time (LSST) . . . . . . . . . 21

3.2 .6 Latin American PhD program . . . . . . . . . . . . . . . . . . . . . . 22

3.3 Areas of Excellence . . . . . . . . . . . . . . . . . . . . . . 23

3.4 Synergies . . . . . . . . . . . . . . . . . . . . . . . . . . . . . . . . . $2 \ldots^{23}$

3.5 Conclusions . . . . . . . . . . . . . . . . . . . . . . . . . . . 24

4 Dark Matter

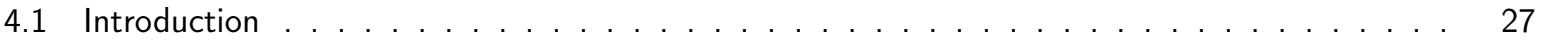

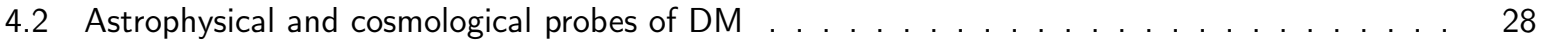

4.2 .1 Direct detection . . . . . . . . . . . . . . . . . . . . . . . . . . . . . 28

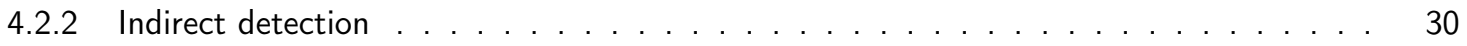

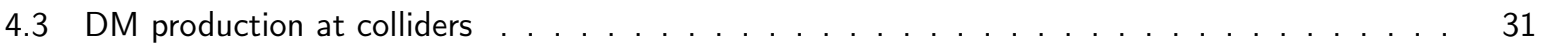

4.4 DM portals . . . . . . . . . . . . . . . . . . . . . . . . . . . . . . . . . . .

4.5 DM Phenomenology community in LA . . . . . . . . . . . . . . . . . . 33

4.6 Synergies . . . . . . . . . . . . . . . . . . . . . . . . . . . . . . . . . . . . . . . .

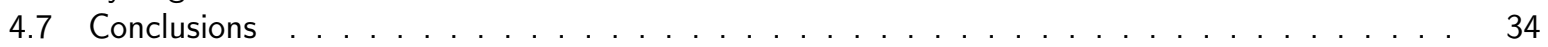

5 Neutrinos 42

5.1 Introduction . . . . . . . . . . . . . . . . . . . 42

5.1.1 Neutrino oscillations, mass hierarchy and leptonic phase . . . . . . . . . . 42

5.1 .2 Neutrino masses and nature . . . . . . . . . . . . . . . . . 44

5.1 .3 Astrophysical probes . . . . . . . . . . . . . . . . . . . . 45

5.1.4 Search for new neutrinos states: light sterile neutrinos and heavy neutral leptons . . . 46

5.2 Research infrastructures . . . . . . . . . . . . . . . . . 47 
5.2.1 Latin America-based large-scale infrastructures . . . . . . . . . . . . . . 48

5.2.2 Latin America-based small-scale - high impact- infrastructures . . . . . . . . . . . 48

5.2 .3 International large-scale infrastructures . . . . . . . . . . . . . . . 50

5.3 Areas of excellence in Latin America . . . . . . . . . . . . . . . . . . . . . . . 52 5

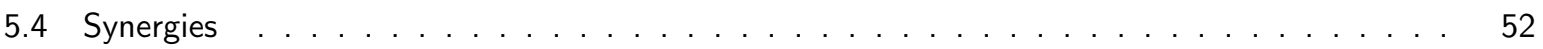

5.4 .1 Local large-scale infrastructures . . . . . . . . . . . . . . . . . . 52

5.4 .2 Local small-scale infrastructures . . . . . . . . . . . . . . . . . . . . 55

5.4 .3 International large-scale infrastructures . . . . . . . . . . . . . . . . 53

5.5 Conclusions . . . . . . . . . . . . . . . . . . . . . . . . . . 53

6 Electroweak \& Strong Interactions, Higgs Physics, CP \& Flavour Physics and BSM 57

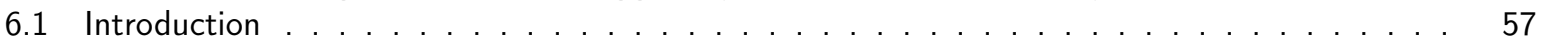

6.2 Participation of LA groups in HEP Activities . . . . . . . . . . . . . . 60

6.2 .1 Nuclear Physics . . . . . . . . . . . . . . . . . . . . . . . . . . . 60

6.2 .2 Jefferson Laboratory . . . . . . . . . . . . . . . . . . . . . . . . 63

6.2 .3 LHC-ATLAS . . . . . . . . . . . . . . . . . . . . . . . . . . . . . . . . . 63

6.2 .4 LHC-CMS . . . . . . . . . . . . . . . . . . . . . . . 65

6.2 .5 LHC-LHCb . . . . . . . . . . . . . . . . . . . . . . . . . . . . . . 66

6.2 .6 LHC-ALICE . . . . . . . . . . . . . . . . . . . . . . . . . . . . . . . 67

6.2 .7 SuperKEKB . . . . . . . . . . . . . . . . . . . . . . . . . . . $6 . \ldots \ldots$

6.2 .8 Future Colliders . . . . . . . . . . . . . . . . . . . . . . . . . . . . . . . 68

6.2 .9 Theory . . . . . . . . . . . . . . . . . . . . . . . . . . . . 69

6.3 Training, outreach, exchange programmes . . . . . . . . . . . . 70

6.4 Areas of excellence and leadership . . . . . . . . . . . . . . . . . . . . . . . . . $\quad \ldots$

6.5 Synergies . . . . . . . . . . . . . . . . . . . . . . . . . . . . . . 70

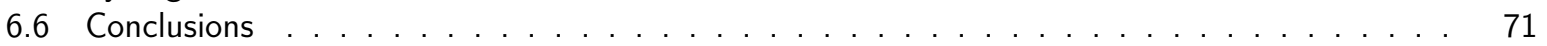

7 Instrumentation and Computing

7.1 Introduction . . . . . . . . . . . . . . . . . 73

7.1 .1 Main key scientific questions and highlights . . . . . . . . . . 73

7.1 .2 Non-scientific drivers . . . . . . . . . . . . . . . . . . . . . . . 75

7.2 Topics within similar instrumentation drivers $\ldots \ldots \ldots \ldots \ldots \ldots \ldots$

7.2 .1 FPGA Boards . . . . . . . . . . . . . . . . . . . . . . . . . . 75

7.2 .2 Read Out systems . . . . . . . . . . . . . . . . . . . . . . 76

7.2 .3 Small-area Photomultipliers (sPMTs) . . . . . . . . . . . . . . . . . . 76

7.2 .4 Silicon Photomultipliers (SiPMs) _. . . . . . . . . . . . . . . . . . 76

7.2 .5 Charge-Coupled Devices (CCDs and Skipper CCDs) . . . . . . . . . . . . . . . . . 77

7.2 .6 Resistive Plate Chambers (RPC) . . . . . . . . . . . . . . . . . . . 77

7.2.7 ARAPUCA Light Trap (Argon R\&D Advanced Program at UNICAMP) . . . . . . . 78

7.2 .8 Water Cherenkov Detectors . . . . . . . . . . . . . . . . . . . . . . . . 78

7.2 .9 Laser Interferometer . . . . . . . . . . . . . . . . . . . . . . 78

7.3 Computing and software . . . . . . . . . . . . . . . . . . . . . . 79

7.3 .1 General remarks . . . . . . . . . . . . . . . . . . . . . . . . . . 79

7.3.2 Large collaboration examples in the region . . . . . . . . . . . . . 79

7.3 .3 Training and knowledge transfer efforts . . . . . . . . . . . . . . . . 80

7.4 Synergies with other chapters/scientific topics . . . . . . . . . . . . . . . . . . 80

7.5 Developing and preserving knowledge and expertise . . . . . . . . . . . . . . 81

7.6 Conclusions . . . . . . . . . . . . . . . . . . . . . . . . 81

8 Appendix 84

8.1 List of White Papers . . . . . . . . . . . . . . . . . . . . . . . . 84

8.2 Glossary of Experiments . . . . . . . . . . . . . . . . . . . . . 85 


\section{Introduction}

For the first time the scientific community working at the forefront of research in high energy, cosmology and astroparticle physics (HECAP) in Latin American have come together to discuss and provide scientific input towards the development of a regional strategy for these fields of knowledge. The process for the creation of a long-term strategy is unfolded in two main avenues: the development of new experiments/facilities in the region and the construction of synergies for a more compelling and coordinated participation in global projects.

The process derived from the October 2018 Iberoamerican Science and Technology Ministerial meeting and its corresponding declaration ${ }^{1}$. This declaration was then ratified at the end of 2018 by the Heads of State ${ }^{2}$ The declaration gave the mandate to create a Strategic Forum for Research Infrastructures, both to provide an incentive for participation in existing facilities as well as to foster the development of new projects and initiatives. It is important to note that in the area of HECAP Spain and Portugal take part in the European processes for the development of the corresponding strategy. Thus, a necessary first step was to pursue the HECAP strategy at the Latin American level that would then interface with those of Spain and Portugal.

The Latin American Strategy Forum for Research Infrastructures (LASF4RI ${ }^{3}$ ) took shape as a result of the first workshop organized at ICTP-SAIFR in Sao Paulo, April 30-May 1st 20194. The decision was then made to move ahead to pioneer the first LASF4RI initiative with HECAP. The LASF4RI-HECAP process began with the establishment of the Preparatory Group (PG) and the High Level Strategy Group (HLSG). The purview of the Preparatory Group is to develop the community input process and related discussions to produce the Physics Briefing Book. Consequently, a broad invitation was made to the Latin American HECAP community to submit their proposals. By December 201940 proposals were submitted, see full list of submissions in section 8.1. The submissions were considered by the members of the Preparatory Group and organized by topic into six working groups as defined below. The next step in the process was to hold an Open Symposium to present the submitted proposals and allow for additional input and discussions among the community members on the outlook for HECAP in the region ${ }^{5}$ This took place in July 2020 with broad participation of the community albeit the distress induced by the pandemic. At the online Open Symposium, there were brief presentations of the submissions, together with a compiled summary per topical working group presented by PG members, in their role a group convenors. Extensive discussion sessions were held for each topic and a final general discussion closed the event. Additional input was sought from the community, when necessary, in order to strengthen and improve on the content and output of the process.

The present document, the Latin American HECAP Physics Briefing Book, is the result of this ambitious grass roots effort. This report contains the work performed by the Preparatory Group to synthesize the main contributions and discussions for each of the topical working groups:

- Astronomy, Astrophysics and Astroparticle Physics

- Cosmology

- Dark Matter

- Neutrinos

- Electroweak and Strong Interactions, Higgs physics, Flavour and CP Physics and Beyond the Standard Model Physics

- Instrumentation and Computing

It is of interest to note that recent work done in Europe to update the European Strategy for Particle Physics summarized in the corresponding Physics Briefing Book (https://arxiv.org/abs/1910.11775) dives into a

\footnotetext{
${ }^{1}$ https://www.segib.org/wp-content/uploads/Declaracion-III-Reunion-de-Ministros-y-Altas-Autoridades-en-CienciaTecnologia-e-Innovacion_ES.pdf

${ }^{2}$ https://www.segib.org/wp-content/uploads/III-PROGRAMA-DE-ACCION.pdf

${ }^{3}$ lasf 4 ri.org

4 www.ictp-saifr.org/workshop-on-the-latin-american-strategy-forum-for-research-infrastructure/

$5_{\text {www . ictp-saifr. org/lasf4ri2020/ }}$
} 
broader and more in depth discussion on the global physics landscape and future, that complements the Latin American regional perspective presented here. Similarly the European Astroparticle Physics Strategy ${ }^{6}$ devised the specific strategy for astroparticle physics for the timeframe 2017-2026. In addition, the US has also started its community based Snowmass decadal process that will inform the corresponding strategy in that nation. This Latin American HECAP Physics Briefing Book is fundamental to inform the Strategy Document Committee in the preparation of the Strategy Document that will provide essential input to the LASF4RI-HECAP HLSG. The main role of the HLSG is to analyze the resulting work of the process and in particular to provide feedback, assess, and validate the Strategy Document. The expectation is that the Strategy Document should be revisited on a 3-5 years timeframe. Nevertheless, the extent of the impact of the COVID-19 pandemic on the timescale is still not clear at the time of writing this report.

As can be seen in the subsequent chapters of this briefing book, for the first time a landscape of the Latin American experimental activities in HECAP is presented in a succinct and combined form. The focus is on the scientific questions addressed by experiments that are either located in Latin America or that have a significant participation of Latin American research groups.

This briefing book discusses the relevant emerging projects developing in the region and considers potentially impactful future initiatives and participation of the Latin American HECAP community in international flagship projects. Identifying synergistic contributions to these endeavors is the main outcome of this work.

The comparative advantages for experimental projects for astroparticle physics and cosmology located in the Latin American region, the increased growth and participation of research groups in HECAP, the strengths and expertise that have been consolidated as well as the remaining shortfalls in capabilities, infraestructure and sustained support are some of the main takeaways of this process. For example, accelerator physics is identified to be an important gap in the existing scientific capabilities in the region.

The second, third and fourth chapters of this briefing book are devoted to the confluence of astronomy, particle physics and cosmology experiments that cover multi messengers of the Universe (visible light, cosmic rays, gamma rays, neutrinos, gravitational waves, etc), precision measurements of the CMB (baryon acoustic oscillations in radio, studies of CMB polarization at large and small angular scales), next generation gravitational waves projects, including also the nature of dark matter/dark energy from galaxy surveys and direct/indirect dark matter detection experiments.

Current and prospective astroparticle and cosmology probes build on the demonstrated ability to host and operate such type of large experiments in the Latin American region and significantly benefit from favorable geographic and atmospheric conditions. The projects under consideration aim to ensure that a leading role on the global scale in this field is maintained in the region. The Latin American participation in direct detection dark matter experiments has a unique potential searching for dark matter candidates in the low mass (sub-GeV) mass region. Impactful participation in liquid Argon detector based experiments and, most recently, demonstrated capabilities based on Skipper-CCD technology offer a clear path ahead. A game-changer opportunity is presented to design and construct experiments based on Skipper-CCD detectors for dark matter and neutrinos in the prospective ANDES facility.

The fifth chapter focuses on neutrino physics in which there is a significant participation of Latin American scientists both in experiments trying to pin down the fundamental nature and properties of neutrinos, as well as in experiments using neutrinos as a probe. There is a wealth of physics domains including precision oscillation measurements, neutrino cross sections, non-standard interactions, CP violation, new neutrino states, astrophysical neutrinos, etc. As examples of forefront technological advancements of great impact for current and future neutrino experiments with Latin American participation are the aforementioned Skipper-CCD detectors as well as the ARAPUCA photon detection system. The latter is a new technology developed in Latin America, that has a central role in detecting the light emitted in detectors at current Fermilab neutrino experiments, and will be instrumental to the prospective Deep Underground Neutrino Experiment (DUNE) physics program. As well as, the planned small photo-multipliers subsystem of JUNO and the optical calibration system for $\mathrm{KM} 3 \mathrm{NeT}$.

Chapter six is devoted to experiments at colliders that probe the fundamental understanding of electroweak and strong interactions at the energy and luminosity frontiers. One of the main features of the last two decades has been the significant growth of the high energy physics community in the Latin American region. This is exemplified by the increased participation of Latin American researchers in all Large Hadron Collier (LHC) experiments, having thus contributed to some of the most outstanding discoveries and measurements related to Higgs physics, the quark gluon plasma, precision electroweak physics, flavour and CP physics. This last topic is

\footnotetext{
${ }^{6}$ (https://www. appec.org/wp-content/uploads/2017/08/APPEC-Strategy-Book-Proof-23-Nov-2.pdf
} 
also complemented by the experimental efforts in Belle 2. At the same time these energy frontier experiments have the potential for new discoveries beyond Standard Model physics that could explain some of the most fundamental unknowns in our understanding of the microscopic world. As a result of these activities clear areas of expertise and excellence emerge that encompasses detector design and construction, triggers, Data Acquisition (DAQ) and readout systems, software and computing, as well as leading certain physics analyses.

The final chapter addresses the area of instrumentation and computing. Future experimental activities with their high specification requirements across HECAP drive the focus of the instrumentation R\&D efforts as well as the evolving needs of computing resources. The Latin American expertise and development of technologies and devices that can be utilized across HECAP is presented including specific chip and board design, firmware and construction, electronic readout systems, photonics, charged coupled devices, Resistive Plate Chamberss and water Cherenkov detectors. The computing infrastructure requirements are also indicated and the case is made for a strategic R\&D computing approach to benefit HECAP in Latin America, including the advanced training, development and retention of expertise.

Throughout the chapters there are important references to advanced training and capacity building which is a strength in the region and is key to increase participation and the visibility of the community in the amazing projects that are considered here.

It is worth mentioning that in all of the physics topics mentioned above the fruitful exchanges between theoretical and experimental physicists have been of great importance to support activities and capability growth, motivate new searches, physics analysis, or the development of new techniques. The main objective of this report is to evaluate the research infrastructures, rather than concentrate on the theoretical aspects of HECAP, although a significant participation of Latin American theorists was noticeable throughout this process. Throughout the previous sections, ample consideration is given to the overlap of the scientific drivers when appropriate, e.g. direct searches for dark matter or new neutrino states at the LHC.

The present Physics Briefing Book of HECAP activities in Latin America aims at providing a landscape of existing expertise and capabilities. It will hopefully instruct some recommendations for further development of strategic research infrastructures relevant for future HECAP efforts in the region as well as for a continuous and successful involvement of Latin American HECAP researchers in the global arena. 


\section{Astronomy, Astrophysics and Astroparticle Physics}

Juan Carlos D'Olivo (UNAM, Mexico)

Federico Sánchez (U. Nacional de San Martín, Argentina)

Martín Subieta (U. Mayor de San Andrés, Bolivia)

\subsection{Introduction}

The subtle connection between the macro- and micro-cosmos is probably the most prominent feature of the astroparticle physics field, which is a research area that in the last decades had an explosive development as demonstrated by the six Nobel Prizes that have been awarded in the last two decades: 2002, 2006, 2011, 2015, 2017, and 2019. Being at the interface of astronomy, particle physics and cosmology, this field will certainly play a major role in pushing our knowledge of the Universe to the edge and, undoubtedly, its prospects for the near future are very exciting ${ }^{1}$

The domain of elementary particles with their fundamental interactions, on one side, and the world of the celestial objects with their evolution, on the other, have established two highly successful standard scenarios: the Standard Model of particle physics (SM) and the Big Bang Model (BBM) of cosmology . Both are playing a crucial role in the current understanding as well as in the unsolved enigmas of our Universe.

The main open questions that can be addressed from astroparticle physics might be summarized in two broad sets. Namely,

- the non-thermal universe: what can be learned from the most energetic messengers (gamma rays, neutrinos, cosmic rays, and gravitational waves) of our universe? What is the link among them? What are the acceleration sites of these high-energy particle and what are the mechanisms involved?

Particles with energy above $10^{9} \mathrm{eV}$ are the messengers of the extreme Universe. In addition to the radiation emitted by stars and produced predominantly by thermal or collisional emission, we know that some forms of radiation are independent of gas temperature and require the action of a collective process of concentration of energy on a small population of particles. The energy spectrum of gamma and cosmic rays, which cannot be reduced to a thermal process, are a good example. We also know that both are indeed an important piece of information in our task to describe the Universe and its fundamental laws since their energy density is of the same order of magnitude as the energy density of stars light and the galactic magnetic fields [3].

Several observatories around the world have inaugurated a new way of observing the Universe by means of cosmic rays, gamma-rays, cosmic neutrinos and gravitational waves. As an example of the connection of all these observations, it has been argued that mergers of black holes, both of stellar mass and of extreme masses, are a key ingredient for producing ultra high energy cosmic rays (UHECRs), high energy neutrinos, high energy photons and gravitational waves. The key recognition is that, during a merger, the individual spins and the accompanying relativistic jets swing around, at full power, until they are aligned with the orbital spin [4] This swerving around of a full power jet system would be directly visible in many observations.

The simultaneous observation of cataclysmic events in our Universe with multiple messengers provides, therefore, new insights into the astrophysical properties of compact objects and also stringent tests of

\footnotetext{
${ }^{1}$ It worth noting that the international community is recently organising itself around these research axes. For example, in 2012, the European community formed the Astroparticle Physics European Consortium (APPEC) as a consortium operated on the basis of a Memorandum of Understanding with the overarching aim of strengthening European astroparticle physics and the community engaged in this field. For more information: https://www.appec.org/
} 
physical laws and particle properties, which additionally contributes to the search for dark matter (DM).

- the dark universe: what is the nature of dark matter? What is the role of tiny mass neutrinos? Do axion-like particles exist?

Unlike normal matter, well explained and understood within the context of the SM, DM does not seems to interact electromagnetically: it does not absorb, reflect or emit light. The existence of DM is inferred only from its gravitational effects, particular at large scales. It may be comprised of particles whose masses span orders of magnitude from $10^{-22} \mathrm{eV}$ to $1 \mathrm{PeV}$ and the most frequently proposed candidates arise in theories that suggest physics beyond the SM, such as supersymmetry and extra-dimensions.

The currently dominating paradigm describes the DM as a relic density of weakly interacting massive particles (WIMPs) in the range $10^{2} \mathrm{GeV}$ to $10^{4} \mathrm{GeV}$. The products of the self-annihilation of WIMPs would interact with the environment at the place of annihilation. The self-annihilation would therefore be more sizable in astrophysical environments, such as galaxies and clusters, richest in dark matter. In fact, the products of DM annihilation would show up, in such objects, as an additional, exotic, gamma component on the top of the astrophysical one. This extra component depends on the nature of the dark matter through its annihilation cross-section, its mass, its couplings to the Standard Model, all eventually affecting the spectrum of gamma-rays emitted. This indirect searches of DM annihilation (or decay) have therefore become one important tool of all Gamma-Ray observatories.

On the other hand, models of superheavy DM (SHDM) that have been proposed since the early 90s [2] are being revived due to the null detection (both direct or indirect) of WIMPs. If super-heavy particles decay into SM particles, a flux of ultra-high energy photons could be observed preferentially from regions of denser DM density such as the center of our Galaxy. So far, no photons with energies above $1 \mathrm{EeV}$ have been unambiguously identified [1]. This can translate into constraints on the properties of the SHDM particles like their mass or lifetime. Ultra-high-energy cosmic rays observatories might, therefore, also contribute to solve the DM mystery.

The Latin American region has an unique-opportunity window to host many large international projects in the field of astroparticle physics. In observational astronomy Latin America is strategically important given its geographical position. The present, the mid- and the long-term future are covered by different proposals. Nowadays, the landscape with the ongoing observatories is bright, but the prospects for the future might be undoubtedly stunning with the proposed projects.

The Pierre Auger Observatory (PAO) [I-17], installed in Argentina since 20 years ago, is the world's largest and most sensitive facility to detect giant particle showers generated by the interaction of high-energy cosmic rays. It has certainly paved the way for other collaborations to come to Latin America. The next generation ground-based gamma-ray observatory, the Cherenkov Telescope Array (CTA) [1-6, I-24], that will be hosted in Chile, is a clear example in this sense. The construction of CTA, which is foreseen to finalize in 2025, will improve the sensitivity, angular resolution, field of view and energy coverage of the current experiments by around one order of magnitude, providing an impressive boost to gamma-ray observations. Another example is the High-Altitude Water Cherenkov Gamma-ray Observatory (HAWC) located in Mexico since 2007, which is the basis for the next-generation Southern Wide-field-of-view Gamma-ray Observatory (SWGO) [I-23] to be hosted in the region. Also important for the region, is the Andes Large-area PArticle detector for Cosmic-ray physics and Astronomy (ALPACA), another gamma ray observatory that will be located very near to Mt. Chacaltaya, in Bolivia.

Cosmology and radio-astronomy experiments have benefited from the crystal-clear skies in the Latin America region: the Q\&U Bolometric Interferometer for Cosmology (QUBIC) [I-37] and the Large Latin American Millimetre Array (LLAMA) are under construction in the north-west of Argentina.

A spin-off of all these efforts is the Latin American Giant Observatory (LAGO) [I-29], a network of detectors distributed all over Latin America. It represents a non-centralized and almost completely regional effort whose purpose is the study of high-energy phenomena, space weather, and atmospheric radiation.

Finally, the prospects for the region to be host of a Giant Radio Array for Neutrino Detection (GRAND) [I-25] show the strong commitment of Latin America to the astroparticle physics field both in developing research facilities and leading them with highly trained human resources.

In short, the infrastructures already deployed, as well as those being constructed, planned or in their design phase, will exploit all confirmed high-energy astroparticle messengers from the Universe and are essential for enabling new, or strengthening the already existing, Latin American scientific capabilities. 
In this chapter the various fields and, particularly, the different observatories in astroparticles most relevant for the Latin American region are briefly reviewed. Before the concluding remarks, some considerations on the synergies with other chapters of the briefing book are also addressed.

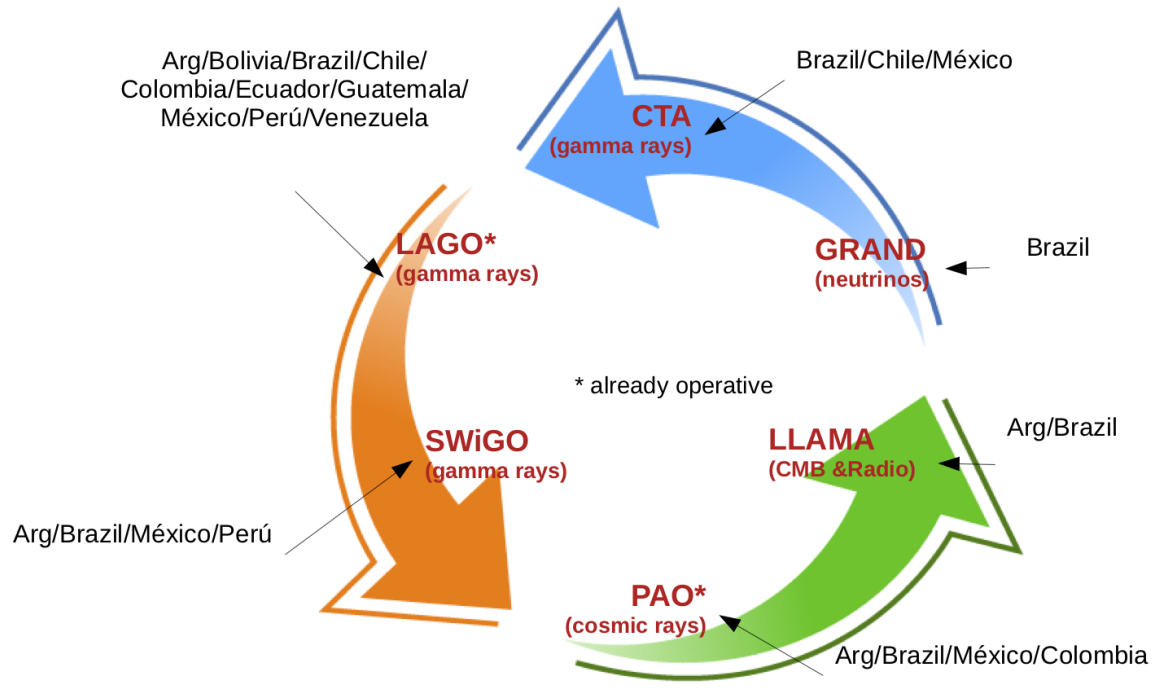

Figure 2.1: Latin American prospect in astroparticle physics. Five out of six large international projects have already selected the Latin American region as host sites. Participation of Latin American countries is also indicated.

\subsection{Involvement of Latin American Countries}

Latin America has a strong commitment in astronomy, astrophysics and astroparticle physics field as demonstrated by the several intercontinental collaborations that decided to place their projects in the region (see Figure 2.1). There are several reasons behind these choices but most of them rely on the well-established comparative advantages with respect to other areas in the world. The access to crystal-clear skies as well as the support of local scientific communities are certainly among the key advantages provided by the countries in the region. The following presentation follows the white papers of the proposals submitted to LASF4RI-HECAP.

\subsubsection{Pierre Auger Observatory}

PAO's main physics goals are:

1. To elucidate the mass composition and the origin of the flux suppression at the highest energies.

2. The search for a flux contribution of protons up to the highest energies ( sensitive up to $10 \%$ fraction of $\mathrm{p}$ in the suppression region).

3. Understanding extensive air showers and hadronic interactions.

4. To probe the origin and characteristics of primary cosmic rays from below $10^{17} \mathrm{eV}$ up to $10^{20} \mathrm{eV}$.

The PAO corresponds to a $3000 \mathrm{~km}^{2}$ area in Mendoza Province (Arg.) embedded with water-Cherenkov detectors, flourescence telescopes, plastic-scintillation detectors, radio-antennas detectors, and resistive-plate chambers. 
The PAO collaboration consists of 80 institutes from 17 countries (Argentina, Australia, Belgium, Brazil, Colombia, Checz Rep., France, Germany, Italy, México, Poland, Portugal, Romania, Slovenia, Spain, Netherlands, USA). The construction finalized in 2008 and since 2015 is constructing the upgrade. The completion of the upgrade is foreseen to be in 2022.

\subsubsection{Latin American Giant Observatory}

LAGO's mains physics goals are:

1. Search for Gamma Ray Bursts (GRBs) events.

2. Study the flux modulation of galactic cosmic rays at different locations on Earth.

3. Study of variation of secondary particles at detection level.

4. Study of the global magnetic structure cloud reaching the space environment surrounding the Earth.

5. Space weather studies.

This widespread network of observatories is deployed throughout Latin America, in sites located at different latitudes, from Mexico to Antarctica, and at different altitudes.LAGO is based on single or small arrays of water-Cherenkov detectors. The LAGO collaboration consists of 29 institutes from 11 countries (Argentina, Bolivia, Brazil, Chile, Colombia, Ecuador, Guatemala, México, Perú, Venezuela, Spain). This network started activities in 2006. The current detector upgrade plan is expected to take place in 2020-2025.

\subsubsection{Cherenkov Telescope Array}

CTA's main physics goals are:

1. Understanding the origin and role of relativistic cosmic particles (sites and mechanism of high-energy particle acceleration).

2. Probing extreme environments (physical processes at work close to neutron stars and black holes, characteristics of relativistic jets, winds and explosions, radiation fields and magnetic fields in cosmic voids).

3. Exploring frontiers in physics (the nature and distribution of dark matter, quantum gravitational effects on photon propagation).

The CTA observatory consists of two array site locations, one in the Southern (Paranal, Chile) and one in the Northern hemisphere (Canary Island, Spain). It will use imaging atmospheric Cherenkov telescopes of three-different kinds: the Small Size telescopes (SSTs), the Middle Size Telescopes (MSTs), and the Large Size Telescopes (LSTs).

The CTA collaboration consists of 200 institutes from 31 countries (Armenia, Australia, Austria, Brazil, Bulgaria, Canada, Chile, Croatia, Checz Rep., Finland, France, Germany, Greece, India, Ireland, Italy, Japan, México, Namibia, Netherlands, Norway, Poland, Slovenia, S. Africa, Spain, Sweden, Switzerland, Thailand, Ukraine, UK, USA). The current timeline foresees completion of construction by 2025.

\subsubsection{Southern Wide-field-of-view Gamma-Ray Observatory}

SWGO's main physics goals are:

1. Measure TeV halos around nearby Pulsar Wind Nebulas.

2. Identify sources of PeV galactic cosmic rays.

3. Measure the galactic center and Fermi Bubbles morphology.

4. Measure the galactic diffuse emission, the local galactic cosmic ray anisotropy and solar cosmic rays interactions.

5. Search for new VHE gamma ray galactic sources and neutrino VHE gamma rays counterparts, detect AGN flares and issue alerts, and search for periodicity and long term emission in AGNs.

6. Search for counterparts to GW events and nearby bright GRBs.

7. Search for dark matter annihilation and decay, Lorentz Invariance violation, primordial black-holes or axion-like particles.

SWGO's site candidates are: Atacama dessert (Chile), Cerro Vecar (Argentina), ALPACA site and Mt. Chacaltaya (Bolivia), Laguna Sibinacocha (Perú). It will be based on water-Cherenkov technique graded in 
4000 detectors deployed in $0.08 \mathrm{~km}^{2}$ and 1000 detectors an extended area of $0.22 \mathrm{~km}^{2}$. The SWGO is foreseen to be completed in 2026. The SWGO collaboration consists of 43 institutes from 10 countries (Argentina, Australia, Brazil, Germany, Italy, México, Perú, Portugal, UK, USA).

\subsubsection{Large Latin American Millimeter Array}

LLAMA's main physics goals are:

1. Astrophysics in multiple wavelengths (solar physics, cosmology, galactic structure, astrochemistry, interstellar Medium, star formation).

2. Observations of atomic molecular spectral lines coming from astronomical objects located in a wide range of distances and surveys of the southern sky for different molecular lines.

3. Very Long Baseline Interferometry (VLBI) observations.

4. Study of the polarization of Cosmic Microwave Background Radiation (CMBR) at small angular scale (complement to QUBIC).

It is under construction in North-Western Argentina (Alto de Chorrillos, Salta Province) at $4800 \mathrm{~m}$ above sea level. The LLAMA is a radio telescope with a single dish of $12 \mathrm{~m}$ diameter operated in the frequency range of 95 to $950 \mathrm{GHz}$. LLAMA is a joint project between Argentinian and Brazilian institutions. Construction planning started in July 2014 after the formal signature of an agreement between the main institutions involved. First light is expected in 3-4 years.

\subsubsection{Giant Radio Array for Neutrino Detection}

GRAND's main physics goals are:

1. Study the origin of the Ultra High Energy Cosmic Rays (UHECRs).

2. Perform neutrino astronomy, study GZK neutrinos and neutrino physics.

3. Detect Ultra High Energy (UHE) neutrinos and gamma rays and discover UHE neutrino point sources.

4. Probe millisecond astrophysical transients (fast radio bursts and giant radio pulses).

5. Map the sky temperature with $\mathrm{mK}$ precision and measure the global signature of the epoch of re-ionization and study the Cosmic Dawn.

The site for the prototype is in China but future sub-arrays might be located in Latin America. GRAND will be a network of air shower ground-detectors based on radio-antennas. 8 institutes from 6 countries (Brazil, China, France, Germany, Netherlands, USA) are working in this project. GRANDProto35 (35 antennas and 24 scintillators) was in commissioning phase and several upgrades are planned spanning a timescale up to 2030s.

\subsection{Leadership Areas}

The area of Ultra-High Energy Cosmic Rays observation is undoubtedly one of the most broadly spanned over in the Latin American region. As one of the first and largest collaborations settled in Latin America, the Pierre Auger Observatory has highly boosted the Latin American astroparticle physics community. In the past two decades, utmost contributions from the Latin American scientist were made. Along the years, many Latin American scientists have been in charge of managing roles in the governance of the International Collaboration or leading scientific analyses. For the current upgrade of the Observatory and its related R\&D program, Latin American countries are leading the construction of the Underground Muon Detector (AMIGA) and the Resistive Plate Chamber array (MARTA). Both playing a major role towards solving the problem referred to as the muon puzzle in extensive air showers: none of the current hadronic interaction models tuned after the last run of the Large Hadron Collider (LHC) is able to correctly describe muon production in atmospheric showers induced by cosmic rays. So far, the most unambiguous experimental evidence of this disagreement was revealed in the analysis of PAO data. Thus, this is certainly one of the most compelling issues to be solved in the upcoming years. It is a key contribution to understand the composition of high-energy cosmic rays and, ultimately, the origin of the most energetic particles in the Universe.

Gamma-Ray observation is also a highly developed area in Latin America. No doubt that the construction and the scientific results of HAWC have greatly contributed to this aim. But it is also undeniable that the construction of CTA and SWGO will foster and strengthen it even further. As an example, in the past 10 years, 
the number of scientists involved in CTA only in Brazil, has grown from three to sixty. The science program that will be covered by CTA and SWGO is very broad and will certainly transform our understanding of the high-energy universe exploring questions of fundamental importance: the origin and role of relativistic cosmic particles, the physics in extreme environments, the physics beyond the standard models. Figure 2.2 shows the differential energy flux sensitivity of CTA and SWGO, in comparison with other experiments.

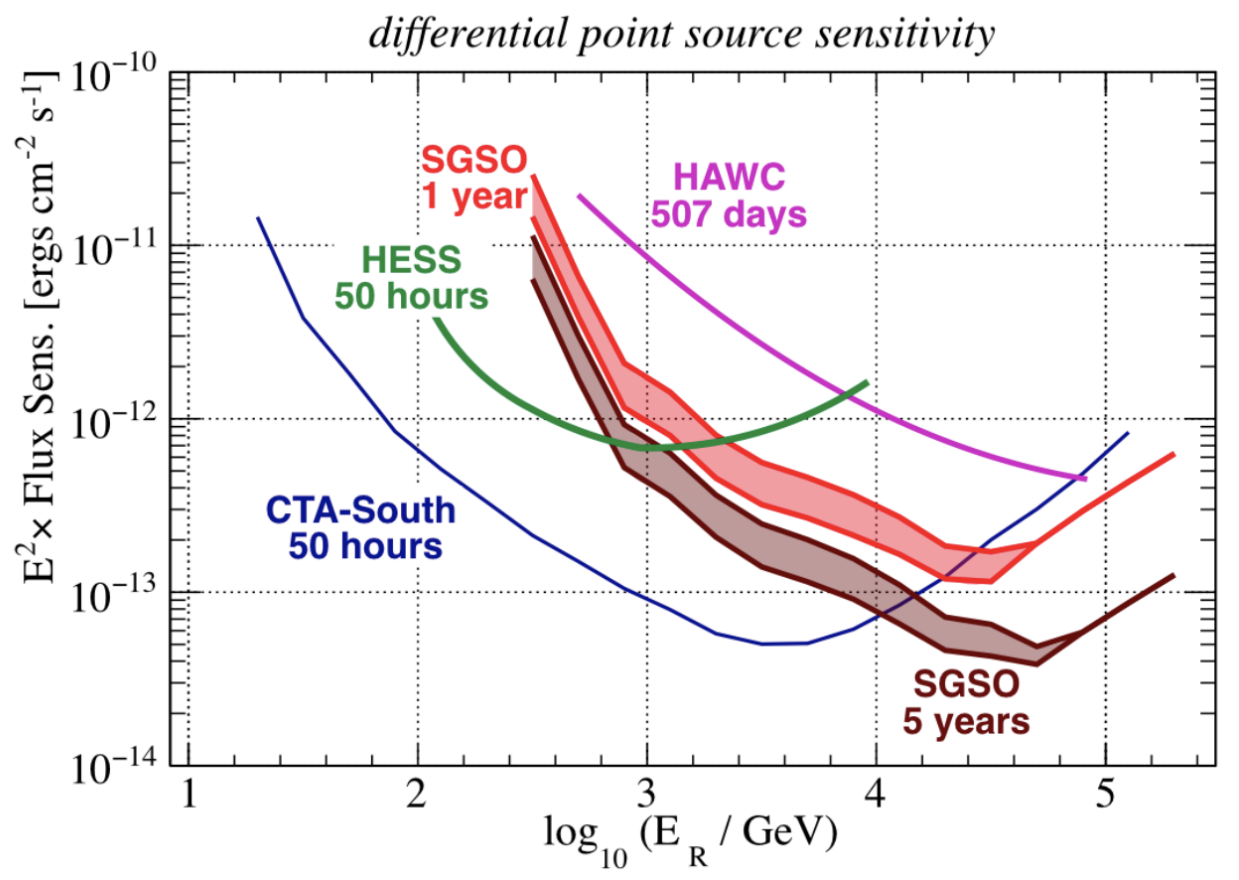

Figure 2.2: CTA and SWGO differential point source sensitivity [5]. Initially SWGO was known as the Southern Gamma-Ray Survey Observatory (SGSO).

Space-Weather studies will benefit from the LAGO project, a spin-off of the Pierre Auger Observatory and a seeder for the development of astroparticle physics in the Andean region. LAGO is rooted in the Latin American region both, for the sites equipped with detectors and for the conformation of the collaboration. LAGO will allow simultaneous measurements of the modulation of the galactic cosmic ray flux at different locations on Earth that can provide important information on the properties and global structure of magnetic clouds reaching the terrestrial environment during interplanetary Coronal Mass Ejections. By combining all the data measured at different locations in the detector network, the LAGO project will provide very detailed and simultaneous information on the temporal evolution and small- and large-scale characteristics of the disturbances produced by different transient and long-term space weather phenomena.

High-Energy Neutrino observations with GRAND benefit from the experience gained, among others efforts in the world, from the PAO engineering array for radio detection (AERA). Since the early 1960s it was suggested that extensive air showers induced by cosmic particles may emit radiation at radio wavelengths. In recent years the interest in this technique has been strongly growing. Besides the already mentioned AERA in the PAO, the activities around the world include LOFAR in the Netherlands, ARIANNA in Antarctica and Tunka-Rex in Siberia. Significant efforts are made to calibrate the response of those detection systems with high precision, both for the signal strength and the signal arrival time. It is worth mentioning thay the cost of deploying radio antennas is lower than for other types of detectors. Moreover, their duty cycle is extremely high and therefore may be a key technique in the near future for observing highly-energetic cosmic radiation. The participation of the Latin American community in GRAND is therefore very important in this line of research. Figure2.3 illustrates the sensitivity to source produced neutrinos at GRAND.

Finally, Experimental Cosmology and Radio Astronomy are also areas in which the Latin American scientific community will play an important role in the coming years. Two experiments, QUBIC and LLAMA, will be conducted in the region. LLAMA will be a multipurpose observatory that will allow observations for long periods of time and explorations of large portions of the sky, impossible to carry out at present through telescopes with 


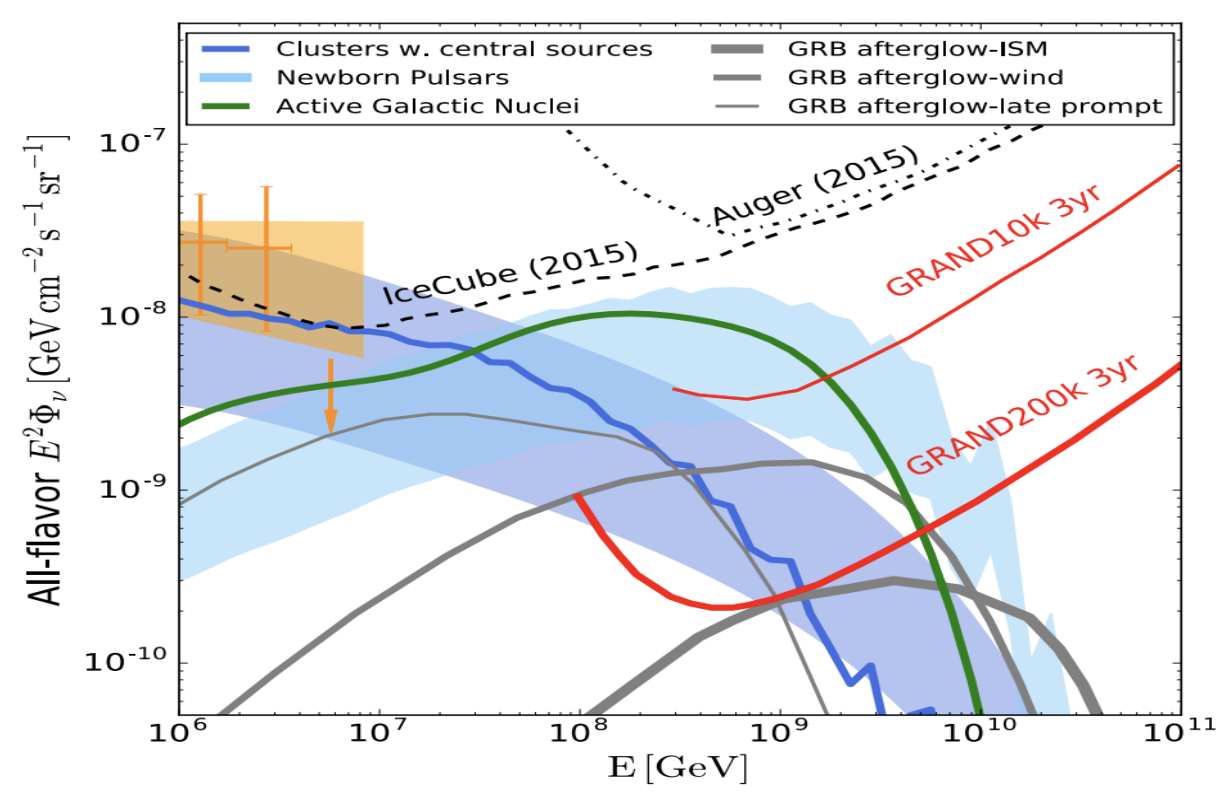

Figure 2.3: GRAND sensitivity to source produced neutrinos (all flavors) [7].

international competition, providing excellent opportunities for groups with interests as diverse as solar physics, cosmology, galactic structure, astrochemistry, interstellar medium, star formation, polarization and magnetism, very large base interferometry, and more. For its part, QUBIC is designed to measure the polarization of the cosmic microwave background and may reveal the presence of gravitational waves with primordial origin. Such measurements can probe inflationary cosmological models that predict that quantum effects during an accelerated expansion at the earliest stages after the Big-Bang produced gravitational waves along with the density fluctuations that later seeded galaxy formation. QUBIC will join other international efforts currently pursuing this goal using a novel approach, which combines the sensitivity of bolometric detectors with the control of systematic effects provided by interferometry ${ }^{2}$.

\subsection{Drivers for Multiple Approaches}

We have recently witnessed the dawn of the era of multi-messenger astrophysics. It was marked by the detection of gravitational waves from a binary neutron star in coincidence with electromagnetic counterparts, and by the observation of high-energy neutrinos from a blazar flare together with radiation across the whole electromagnetic spectrum [8 9$]$. Evidently multi-messenger exploration of the extreme Universe is in full swing alongside the quest for Dark Matter and the search for the true nature of tiny mass neutrinos.

Cosmic ray detectors, gamma ray and high-energy neutrino observatories will benefit each other. The Latin American scientific community has the unique opportunity to host several projects covering all these ways of exploring the Universe and, therefore, significantly contributing to push forward our knowledge of the Cosmos and its evolution.

\subsection{Synergies}

As already demonstrated by previous experiences around the world, there are multiple synergies between particle and astroparticle physics at various levels. From the point of view of infrastructures, large underground facilities

\footnotetext{
${ }^{2}$ Recent information on a new projet was submitted, the Next Generation Very Large Array (ngVLA) will be constituted by 256 antennas of 18-m each distributed across a region $300 \mathrm{~lm}$ wide. It will have one order of magnitude better angular resolution and sensitivity than the VLA. The ngVLA will be distributed in the south of the USA and the north of Mexico. This distribution will provide optimal images of southern sources such as the center of our Galaxy. The radio astronomical community of Mexico is very interested in participating in this project that will have as major partners the USA, Canada, Germany and Japan. It will be very positive to involve other Latin American countries in this collaboration. Even a few percent of guaranteed telescope time will allow the Latin American groups to undertake major projects, given the speed of the instrument.
} 
or the engineering and management of large projects are good examples. From the point of view of detectors and enabling technologies, several R\&D programs including photosensors, electronics or computing are also a taste of how basic astrophysical research strengthens the link between science and technology (see Chapter 7). From both the theoretical and phenomenological side and the fundamental unknowns, there is a large overlap between particle and astroparticle physics:

- The excess of muons with respect to simulated air showers, first observed in 2000, is a good example. While the experimental measurements are consistent within their uncertainties with predictions up to energies of approximately $100 \mathrm{PeV}$, corresponding to proton-air collisions at nucleon-nucleon centre-ofmass energies of $\sqrt{s_{N N}}<14 \mathrm{TeV}$, at higher energies a muon excess is observed that systematically increases with the shower energy for all hadronic interaction models. The main challenge in the description of muon production in the atmosphere is the treatment of hadron-ion interactions in the forward region over many orders of magnitude in energy. Conventional options to improve the description of muon production in hadronic models are the enhancement of the production of baryon-pairs, neutral rho mesons, or heavy quarks, as well as possibly missing physics in the soft-QCD regime of hadron-ion collisions, or other new phenomena. Ongoing studies on the muon discrepancy at the LHC include detailed measurements of prompt hadron production cross-sections in the forward region in proton-proton and proton-lead collisions and measurements of the ratio of electromagnetic and hadronic energy flows, to which the production of $\mathrm{GeV}$ muons is particularly sensitive. Similar studies at central rapidities allowed to improve the hadronic models with the first LHC data. Running the LHC with oxygen beams is planned for 2023, which was largely motivated by the muon excess and the need to study the nuclear modification of hadron production in the first collisions in EAS. Also ongoing is an upgrade of the SMOG system in LHCb, which will extend the unique capability of LHCb to perform fixed target experiments with LHC beams to study late interactions in extensive air showers.

- Another aspect is the contribution of gamma ray observations to solving the question of the nature of the dark matter. In particular, SWGO will scrutinize nearby extended sources of dark matter for evidence of gamma rays produced in annihilation or decay processes. Weakly interacting massive particles (WIMPs) that were once in thermal equilibrium in the early universe remain one of the most promising explanations for dark matter. Thermal WIMPs with masses between $\sim 2 \mathrm{TeV}$ and $\sim 100 \mathrm{TeV}$ are largely still viable, and only astrophysical experiments can probe heavy dark matter $(>1 \mathrm{TeV})$. CTA will probe thermal WIMPs from $100 \mathrm{GeV}$ to $10 \mathrm{TeV}$, leaving the heaviest of thermal WIMP masses unconstrained. SWGO, on the other hand, will be able to probe almost the entire thermal mass range of WIMPs by extending the sensitivity up to $100 \mathrm{TeV}$. Also, both SWGO and CTA will be sensitive to thermal WIMPs with masses from about 1 to $10 \mathrm{TeV}$. If there is a gamma-ray signal in that mass range, both experiments should see it, leading to independent confirmation of the signal. See Chapter 4 for further discussion. Concomitantly to the exploration of the complete WIMP parameter space with gamma ray observatories, the search might be also extended to cosmic ray observatories like PAO by means of the limits (if not detected) of ultra-high energy photons that test the SHDM models.

- The ultra-high-energy neutrinos that might be observed by ground-based experiments like GRAND, are also a probe of fundamental physics. Specific signatures in neutrino-related observables, namely energy spectrum, angular distribution, and flavor ratios, are sensitive to the search for new physics. In addition, neutrino observatories can extend the measurements of the cross section for neutrino-nucleon interactions, see Chapter 5 for further discussion.

Finally, beyond the link with particle physics of most of the projects presented in this chapter, there is also a synergy with cosmology as LLAMA will contribute to this field by means of measuring the polarization of the CMB (see Chapter 3). The specific contribution will be on smaller scales (arcmin or smaller) for the same part of the sky scanned by QUBIC.

\subsection{Conclusions}

The fields of astronomy, astrophysics and astroparticle physics have shown, in the past decades, that the Latin American community has the capacity to conceive, design and execute large scientific projects that reinforce the connection between basic science and technological applications, favoring the progress of the region both in research and innovation. Local support, together with the geographical features of the Latin American region, have played a decisive role for the selection of Latin America as a hub for hosting project managed by large intercontinental collaborations. In this chapter, we have revised the already established experiments, those 
being constructed and finally those projected in astroparticle physics. The number of projects and the people involved in them, place the Latin American community in a leading position in the area beyond any doubt. We are aware that to foster the regional academic community, strengthen Latin American science and technology capabilities, and consolidate the research-industry link, it is ideal to host such large projects. The development of capacities and enabling technologies are also key ingredients for social progress. The revenue generated by hosting this kind of efforts highly exceeds the investment by individual countries. The Latin American region has both an enthusiastic scientific community as well as geographic advantages (clear skies, large flat areas with low anthropogenic noise, etc.) to support these kinds of endeavors. 


\section{Bibliography}

[1] P. A. Collaboration, "Search for photons with energies above 1018 ev using the hybrid detector of the pierre auger observatory," JCAP, vol. 04, p. 009, 2017.

[2] J. L. L. J. R. Ellis and D. V. Nanopoulos, "Confinement of fractional charges yields integer charged relics in string models," Phys. Lett. B, vol. 247, p. 257, 1990.

[3] J. Wdowczyk and A. W. Wolfendale, "Highest Energy Cosmic Rays," Annual Review of Nuclear and Particle Science, vol. 39, pp. 43-71, 1989.

[4] G. L. and P. Biermann, "Supermassive black hole mergers," APJ, vol. 697, pp. 1621-1633, 2009.

[5] A. Albert and et al., "Science Case for a Wide Field-of-View Very-High-Energy Gamma-Ray Observatory in the Southern Hemisphere," arXiv e-prints, p. arXiv:1902.08429, Feb. 2019.

[6] "Latin American Strategy Forum for Research Infrastructure," https://www.lasf4ri.org.

[7] R. Alves Batista and et al., "Open questions in cosmic-ray research at ultrahigh energies," Frontiers in Astronomy and Space Sciences, vol. 6, p. 23, June 2019.

[8] B. Abbott and et al., "Observation of gravitational waves from a binary black hole merger," Physical Review Letters, vol. 116, Feb 2016.

[9] M. Aartsen and et al., "Multimessenger observations of a flaring blazar coincident with high-energy neutrino icecube-170922a," Science, vol. 361, p. eaat1378, Jul 2018. 


\section{Cosmology}

Diana López Nacir (DF/IFIBA UBA-CONICET, Argentina)

Marcelle Soares-Santos (U Michigan, USA)

Thiago Gonçalves (Valongo Observatory, Brazil)

\subsection{Introduction}

Cosmology is a branch of physics and astronomy, dedicated to understanding the global properties and evolution of our Universe. It is a rapidly evolving field that stands at the interface between theoretical physics, particle physics, and astrophysics. Recent advances in both observational capabilities and theoretical understanding has lead to exhilarating deep connections between fundamental physics and cosmology.

As a result of a worldwide community effort, a standard cosmological model has been built. The main pillars of the model are the standard theory of gravity, general relativity (GR), the Standard Model (SM) of particle physics, and the standard physical laws. In standard cosmology, the evolution of the Universe is described starting from a homogeneous and isotropic background Universe with small inhomogeneities characterized by certain statistical distribution. The base model, known as $\Lambda \mathrm{CDM}$, has only six free parameters that can be determined from several different observations.

Remarkably, the success of the $\Lambda$ CDM model requires the existence of Dark Matter (DM), a mysterious component for which there is no fundamental particle inside the SM of particle physics. Therefore, to explain the evolution of the Universe new physics is required. The DM species constitutes roughly $27 \%$ of the current energy budget of the Universe. In contrast the ordinary matter and radiation in the SM corresponds to just the $5 \%$ of the budget. The rest of the energy is described with the so-called cosmological constant $\Lambda$.

The main reason for the introduction of $\Lambda$, as a free parameter in the theory, is that its value can be adjusted to describe the acceleration of the late time expansion of the Universe. There is no fundamental explanation nor understanding for the inferred particular value. While this provides a simple model that is consistent with current observational data, other models provide alternative explanations of this acceleration. For example, some models attribute the acceleration to the presence of a dynamical component known as Dark Energy $(\mathrm{DE})$, and others to a modification of the gravitational laws on cosmological distances. A scientific driver of the community is to understand to what extent it is possible to discriminate among the different models from observations, and whether any of the models are better at fitting the data than what is currently the most accepted explanation, $\Lambda$.

Another mystery of the current understanding of the evolution of the Universe, is the origin of the tiny inhomogeneities in the matter density. Standard theory can explain the formation of structures (such as galaxies, galaxy clusters, etc.) as a consequence of the growth of smaller inhomogeneities in the matter density, but it does not explain the origin of the inhomogeneities. It has turned out to be necessary to assume certain (very particular) properties of the small perturbations that describe the initial departures of a spatially homogeneous and isotropic Universe, that is, of the primordial cosmological perturbations, that are inexplicable within the strict framework of the model. Indeed, the model does not explain why the Universe we observe is so homogeneous and isotropic at "large" distance scales (as inferred from observations of the cosmic background radiation, see below), why large abundances of topological defects such as magnetic monopoles or cosmic strings are not observed, and why the Universe is practically spatially flat. Currently, the most widely accepted solution to these problems is given by the inflationary mechanism. Basically, this consists of adding a period of exponential or quasi-exponential expansion of the Universe, prior to what is known as the hot big-bang Universe. The challenge of explaining the physics of inflation is considerable. Inflation is assumed to have occurred at 
a huge energy scale (perhaps as high as $\sim 10^{15} \mathrm{GeV}$ ), well out of reach of ground particle accelerators. Any description of the inflationary epoch, therefore, requires a huge extrapolation from the known laws of physics.

The Cosmic Microwave Background (CMB) radiation, provides very valuable information about the different parameters that describe the Universe on large scales (among which are the ones that determine the current expansion rate of the Universe $H_{0}$, the relative abundance of matter, radiation, DM and the constant $\Lambda$, as well as those that characterize the primordial perturbations mentioned above and give us information about the inflationary model). The next frontier of $\mathrm{CMB}$ research involves measuring its polarization. After the first detection in 2002, measurements of the CMB polarization have further confirmed the standard cosmological model and have increased the precision on the determination of cosmological parameters.

Observational cosmology has undergone extraordinary advances in the past decade. Nowadays, there are surveys characterizing the large scale structure (LSS) of the Universe by observing galaxies at different frequencies, taking their spectrum, creating catalogs with different properties. For instance, numerous experiments have measured with high precision the so called Baryon Acoustic Oscillations (BAO) (which is a well understood feature in the correlation function of a tracer of the LSS), using both galaxies and the Lyman- $\alpha$ forest as tracers. Current data from (Stage III) spectroscopic surveys already provide constraints of comparable precision to those from the CMB, for some parameters (see for instance [1]).

With the increase of precision, some evidence for discrepancies in the values of the basic parameters have emerged. Primarily when the inferred values for the Hubble constant $H_{0}$ are compared with more direct measurements such as from supernovae catalogs or the strong lensing effect. Depending on the data sets and their combinations, the discrepancy ranges between $4 \sigma$ and $5 \sigma$ significance [2]. Other challenges of the standard paradigm concern the description of some observables on "small" scales, such as the "missing" DM sub-halos or the so called core-cusp discrepancy [3].

From an observational perspective, the current status highlights the importance of having different probes, studying cross correlations between different observables.

Recently, radio surveys are starting to implement a technique called Intensity Mapping (IM). This takes the integrated radio emission from unresolved gas clouds, and uses the redshifted $21 \mathrm{~cm}$ line of Neutral Hydrogen gas ( $\mathrm{HI})$ (observed at radio frequencies) as a tracer of the distribution of the LSS. They are also detecting mysterious Fast Radio Bursts (FRBs) which may be useful for cosmology or for some discovery.

The cosmological gravitational waves (GWs) are another important prediction of inflationary models and can also leave their imprint in the CMB polarization. Whether there is a fraction of the polarization that was caused by GWs or not could be disentangled by using a decomposition of the polarization properties into Emodes and B-modes. Scalar (density or temperature) inhomogeneities, at first order, generate only E-modes. Tensor (GWs) perturbations can produce both. The amplitude of the E-mode signals are much larger than the expected ones for B-modes. Beyond the first order, B-modes can be generated from scalar inhomogeneities, by an effect known as gravitational lensing, which rotates primordial E-modes into B-modes. These B-modes (called "secondary B-modes") have been observed in the CMB polarization at relatively small angular scales, on the order of a few arc minutes. These successful measurements provide an accurate mapping of the gravitational lensing effect which can be used to study the distribution of dark matter on large scales, and may even allow an indirect measurement of the mass of the neutrinos. In contrast, the so called primordial B-modes, imprinted by GWs have not been observed yet. They are expected to peak at an angular scale on the order of one degree. Therefore, a degree scale B-mode polarization signal could be an indirect evidence for the existence of GWs with cosmological wavelengths. From a specific inflationary model, together with a model for the subsequent cosmological history, one can predict the expected level for the amplitude of the signal in B-modes. To characterize the different predictions, a parameter $r$, known as the "tensor-to-scalar ratio", is defined. Therefore, measurements and constraints on $r$ can be used to probe inflationary scenarios. The study of the generation of primordial GWs during inflation has received increasing attention in recent times. Beyond the context of inflation, GWs can be produced in the early Universe by other mechanisms, such as by strong phase transitions after inflation or in non-inflationary alternative scenarios.

By itself, the detection of GWs is an event of spectacular historical importance. GWs are a central prediction of General Relativity, and we have had indirect experimental evidence of their existence for many years: in particular, the Hulse-Taylor binary pulsar PSR B1913 + 16, discovered in 1974, is gradually losing orbital energy at a rate that can be predicted based on gravitational radiation losses. A direct evidence has been the observation of GWs coming from the coalescence of a binary black hole system, carried out in 2016 by LIGO. What this represents for physics is of enormous importance, since it allows the exploration of astrophysical 
objects that would otherwise be invisible, as well as the study of the physical phenomena associated with production of the waves and the corresponding theories.

The beginning of the multi-messenger astronomy era including GWs started with the detection of the GW together with the coincident Gamma Ray Burst (GRB) and other electromagnetic signals, associated to the coalescence of a binary of two neutron stars [4]. The combination of GW detections with electromagnetic-wave astronomy and the study of high-energy particles opened an unprecedented window to probe the Universe. This allows new bounds on the violation of Lorentz invariance, and tests the equivalence principle by constraining the Shapiro delay between gravitational and electromagnetic radiation, as well as constrains on alternative theories of gravity and on models of the dark sector.

From the theoretical perspective, the current status highlights the need of analyzing data to obtain physical quantities, test robustness, to deepen the understanding of data and how they are analyzed, to connect results from cosmological, astrophysical and laboratory experiments, to study alternative theories, their consistency, robustness, connections with particle physics and with the fundamental physics laws, to evaluate the validity of the standard assumptions, etc.

The main science drivers (SD) of the topic, can be summarized in the following questions:

What are the nature, properties and origin of the dark components? Thanks to many observations, not only cosmological but also on astrophysical scales, it has been possible to infer some properties of the dark components. All observational evidence for the dark components is due to its direct or indirect gravitational effects. Their nature is still a mystery, and so far there has been no convincing detection of their possible non-gravitational interactions.

What is the origin of matter-antimatter asymmetry? All visible matter in the Universe is made of fundamental building blocks, the elementary particles. For each elementary particle, there is an antiparticle that has the same properties but opposite charge. In principle, the Big Bang produced equal numbers of particles and antiparticles. However, the Universe today is considered to consist almost entirely of matter (particles) rather than antimatter (antiparticles). The reason why there is matter, and no antimatter, in the energy budget of the Universe is still a mystery. Where have all the antiparticles gone? Currently there is no acceptable understanding of this asymmetry problem.

What are the nature and the properties of neutrinos? As overviewed in [5], one of the great mysteries in particle physics is the determination of the neutrino mass and its fundamental nature. The possibility that neutrinos could be their own antiparticles may be linked to the matter-antimatter asymmetry problem. A cosmological neutrino background is a predicted relic of the standard cosmological model. Although it has not been directly detected yet, it has been indirectly verified by its role in the prediction of the observed primordial abundance of light elements (known as Big Bang Nucleosynthesis process) and by its imprints on the CMB and the LSS. Cosmological data such as CMB and the distribution of LSS are sensitive to the total mass of the neutrinos. From cosmological observations it is also possible to infer the number of relativistic neutrino states in thermal equilibrium in the early Universe, which in standard cosmology it is consistent with three neutrino species, and in general sets further constrains on alternative models and on light sterile neutrino scenarios.

What are the nature and properties of Black Holes (BHs)? This addresses questions such as: Do primordial BHs exist? Are they (part of) the DM? How can BH catalogs be used to address these questions and/or to extract useful cosmological information?

What is the origin and nature of the primordial perturbations? Observations have made it possible to infer many of the statistical properties of primordial perturbations; for example, they are currently compatible with a primordial gaussian distribution of only adiabatic density perturbations and, furthermore, it is possible to set bounds on the level of non-Gaussianities and on the level of primordial GWs. As a result, many of the inflationary models have been ruled out.

Are the standard assumptions wrong? For instance, one can replace GR by another theory of gravity, consider theories were Lorentz symmetry is violated, non-standard thermal or non-thermal history, etc.

\subsection{Experiments and infrastructure with cosmological impact with LA participation}

In this Section we briefly describe the LA participation in some existing and planned experiments as well as some infrastructure and training that have an impact in the area of cosmology. 


\subsubsection{BAO from Integrated Neutral Gas Observations (BINGO)}

The BINGO telescope is a new instrument designed specifically for observing BAO in the frequency band $960-1260 \mathrm{MHz}$ and to provide a new insight into the Universe at $0.13 \leq z \leq 0.45$ with a dedicated instrument. The optical configuration consists of a compact, two $40 \mathrm{~m}$ diameter, static dishes with an exceptionally wide field-of-view $\left(15^{\circ} \times 7^{\circ}\right)$ and 28 feed horns in the focal plane.

BAO observations will be carried through IM HI surveys and its main scientific goal is to claim the first BAO detection in radio in this redshift range, mapping the 3-D distribution of $\mathrm{HI}$, yielding a fundamental contribution to the study of DE, with observations spanning several years. No detection of BAOs in radio has been claimed so far, making BINGO an interesting and appealing instrument in the years to come. Moreover, in view of the observation strategy and with some adjustment in its digital backend, BINGO will also be capable to detect transient phenomena at very short time scales ( $\lesssim 1 \mathrm{~ms})$, such as pulsars and FRBs.

Current LA Involvement: BINGO has currently about 50 participants in more than 20 institutions from Brazil (with the most participants, approximately 30), China, France, Germany, Portugal, Spain, South Africa, Switzerland and England. The project is led by Brazilian researchers, with most of the investment from Brazilian funding agencies (see below), and will be hosted in Brazilian territory.

Facilities and resources: BINGO is being built in Paraíba, Northeastern Brazil, with a strong support of state authorities. There is also support from the Ministry of Science and Technology and FINEP, a related funding agency.

Technological advances: Receiver construction, operating at room temperature, but with a careful frontend design, delivering a very low system temperature and very good stabilization. This subsystem is under development and can be considered a technological advance.

The optical design was mostly done in Brazil, with a complex focal plane distribution of the horns. BINGO is probably the largest example of a "single dish, many horns" telescope. The fabrication of the two 40m dishes are being carried by a Brazilian company, with more than 30 years of experience in telecommunications.

Advanced training: There are currently three Brazilian postdocs and a number of M.Sc. and Ph.D. students deeply involved with aspects of the mission preparation, including development of the necessary instrumentation for the telescope. They are either leading or participating in the first series of papers of the instrument, to be submitted to Astronomy \& Astrophysics not later than October 2020.

Connection with Industries: As mentioned before, the construction of the instrument is led by Brazilian institutions and in being carried by Brazilian companies. As of the time of this writing, there are no spin-offs or start-ups from BINGO.

Timeline and Major Milestones: BINGO will start operations in late 2021. Commissioning of a few receivers operating in the telescope should start by early 2022. Currently the engineering projects for telescope construction are being completed and the terrain is being prepared for construction.

The project has undergone a major review in July 2019, with the some recommendations that include the receiver configuration, final optical design and an "end-to-end" pipeline to simulate the mission behaviour prior to the beginning of operations. The project is within schedule to commence operations as planned.

\subsubsection{Macon Ridge Astronomical Site: The ABRAS and TOROS projects}

The goal is to develop a new astronomical site at the Macon Ridge, in Salta, Argentina (in the Atacama Plateau region) starting with two complementary projects: The Transient Optical Robotic Observatory of the South (TOROS) and The Argentina-Brasil Astronomical Center (ABRAS) projects.

The main scientific goal concerns GW transient events with a main focus on those arising from the coalescence of BNS systems, for which an electromagnetic (EM) follow-up was shown to be promising. For the coalescence of a binary of two Black Holes (BBHs), the most frequently detected events thus far, no EM emission is expected. Nevertheless, exceptional scenarios are being considered for which an EM signal could be detected. There are still clear uncertainties as to the frequency of these events and the improvements in search methods and the progress in the commissioning and final operation of the detectors undergoing through the planned upgrades.

The TOROS collaboration was involved in the EM follow up of the first GW detection of several BBH mergers discovered during the $\mathrm{O} 2$ observational campaign (for which there was no significant EM counterpart found). For the first GW transient associated to a BNS coalescence, GW170817, the collaboration obtained photometry 
for two nights [6]. As for GW170817, the localization of gravitational waves by the network of three detectors is expected to be of a few tens of square degrees. Thus the EM observations need to deal with a rather large positional uncertainty. Figure 3.1 shows the expected light curve of the EM counterpart of a BNS merger at a distance of $200 \mathrm{Mpc}$, as well as the signal-to-noise ratio of the resulting TOROS photometry.
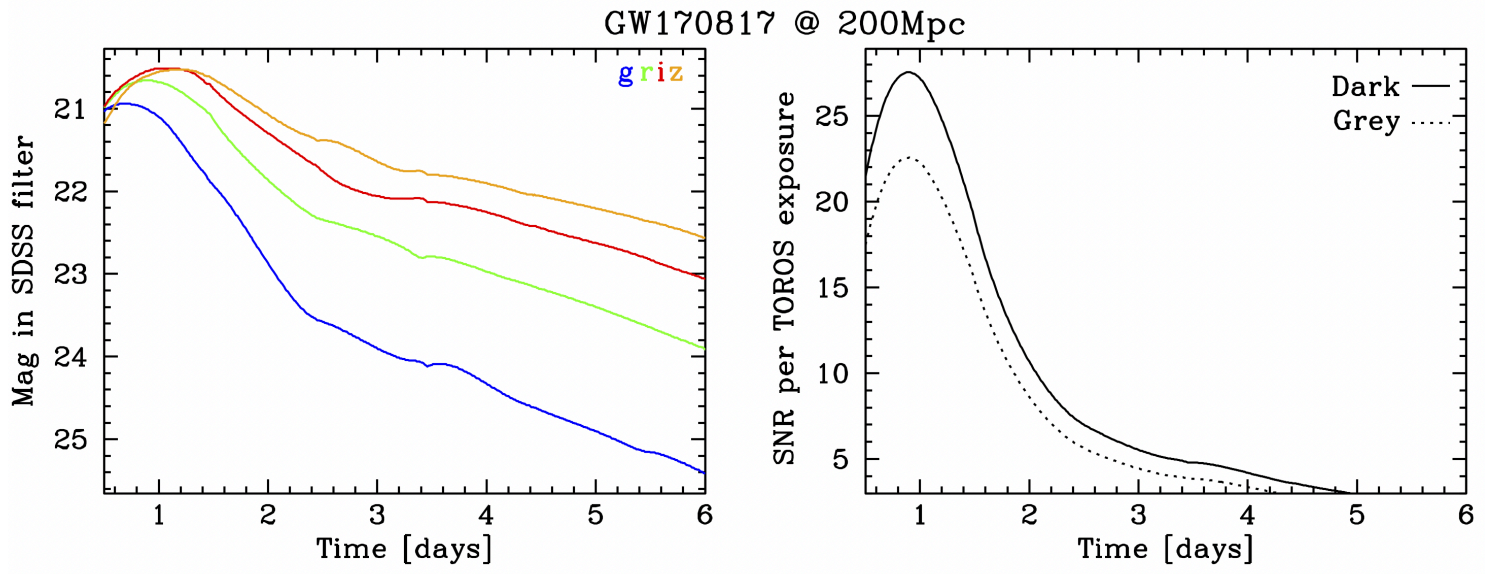

Figure 3.1: Left: light curves of GW170817 77 shifted to a distance of $200 \mathrm{Mpc}$. Right: expected SNR of TOROS photometry of such an event. "Dark" and "grey" refer to typical sky brightness values associated with the new and quarter Moon, respectively. Even in the most pessimistic case, TOROS remains sensitive $(\mathrm{SNR}>3)$ to kilonova events for at least 4 days. Figure reproduced from [8].

The TOROS project will be a fully robotic facility which will offer an extended coverage of the southern skies. The envisioned operation modes, in decreasing order of priority, are: 1) Follow up of gravitational-wave triggers, releasing coordinates of potential transients through the GCN circular methods 1 , and stacked images to the entire astronomical community; 2) Follow up of short-duration $\gamma$-ray bursts (GRBs) events that will serve as GW triggers by performing a similar strategy used to detect the GW counterparts. In the northern hemisphere, this search has started in the last few years by the Palomar Transient Factory and the Zwicky Transient; 3) Baseline imaging of the entire surveyable area (expected to be completed within the first year of operation) to have previous imaging of any region of interest as well as previous knowledge of the rates of transients and variable sources with similar temporal characteristics, useful for estimating the foreground/background contamination rate from other astrophysical events.

The ABRAS telescope will complement TOROS detector at design sensitivity, by carrying out both near-infrared imaging and optical spectroscopic follow-up characterization of transients identified by TOROS.

Current LA involvement: TOROS involves participation from 3 Latin American countries, USA and Poland. In the submitted white paper [8] there are 9 researchers in Argentina (8 members from IATE and 1 interested - not a member - from CASLEO), 4 members in Chile ( 3 from Universidad de La Serena and 1 from Pontificia Universidad Catolica), 2 members in Mexico (1 from Instituto Nacional de Astrofisica and 1 from Optica y Electronica) and 8 members from non-LA countries ( 7 from USA and 1 from In Poland).

ABRAS involves Argentina and Brazil. The main institutions behind are IATE and the IAG (Instituto de Astronomía, Geofísica e Ciencias Atmosféricas), from Sao Paulo, Brasil, while the funding institutions are the MINCyT (Ministerio de Ciencia, Tecnología e Innovación Productiva) and the USP (Universidade de Sao Paulo). The infrared-optimized 1 meter telescope was delivered to Córdoba (Argentina) in late 2015, and the dome was built in Macon Ridge in 2012.

Local facilities and resources: The Astronomical Site and Dark Sky Preserve was established by the Governor of Salta Province in 2011. The preserve is managed by IATE. Roads, electricity and Internet access are available. The local collaboration also involves site characterization, infrastructure, logistics, maintenance and operation. Telescopes at the site can be remotely controlled from village of Tolar Grande $(9 \mathrm{kms}$ distance along the line of the sight). The provincial government constructed a road from Highway 27, which joins Tolar Grande with San Antonio de los Cobres highway, to the Macon ridge.

\footnotetext{
${ }^{1}$ Details about the GCN system are available from http://gcn.gsfc.nasa.gov
} 
TOROS plans to execute their own photometric and spectroscopic follow up of the GW triggers using the Bosque Alegre 1.5- $\mathrm{m}$ telescope in Córdoba and additional resources from the members of the TOROS collaboration in Chile and Argentina (CASLEO observatory in San Juan, Argentina and the Mammalluca observatory from La Serena University in Chile). The spectroscopic followup will be conducted using the ABRAS telescope but also the Gemini, Gran TeCANVLT 8-m telescopes, through target-of-opportunity time to be obtained via the US share of Gemini, and by their Chilean and Mexican collaborators with access to the other facilities.

Technological advances: The considerations sketched above led the collaboration to designe a system with the largest possible aperture within the budget restrictions (primary mirror diameter of $0.6 \mathrm{~m}$ ) and a large field-of-view (10 square degrees) camera with a very broad bandpass $(0.4-0.9 \mu \mathrm{m}$, equivalent to a combination of the Sloan griz filters). There are no commercially available telescope systems (or specific corrector optics) that can meet TOROS requirements. Therefore, a custom corrector optical assembly will replace the secondary structure on the optical tube and will provide the interface between the primary mirror and the CCD focal plane.

Timeline and major milestones: The TOROS telescope is ready to start operations. If funding is secured, the ABRAS telescope could start operations soon. The telescope and its dome have been installed at the site. The camera and the manufacture of the prime-focus corrector are already under construction with commissioning expected by December 2020. The focal corrector is currently under construction and the CCD has already been purchased with expected delivery in 2020 .

\subsubsection{Q\&U Bolometric Interferometer for Cosmology (QUBIC)}

QUBIC is an experiment designed to measure the polarization of the CMB [9]. The main scientific goal is the measurement of a primordial B-modes signal. This is a smoking gun for primordial gravitational waves whose relative strength is given by $r$. The current bound is $r<0.06$ [10], which already disfavours the simplest inflationary models of the early Universe. In the near future, experiments are expected to achieve a sensitivity to $r$ of order $\sigma(r)=0.01$, with improvements up to a few times 0.001. QUBIC is one of the international efforts currently pursuing this goal, and is using a novel kind of instrument, which combines the extreme sensitivity of two arrays of bolometric detectors, operating respectively at $150 \mathrm{GHz}$ and $220 \mathrm{GHz}$, with the control of the systematics offered by the interferometric operation of the instrument.

At the unprecedented sensitivity level that the new generation of experiments aim to reach, the systematic effects control and the possibility to remove foregrounds is a major concern. The primordial B-mode signal can be affected by instrumental systematic effects and is contaminated by astrophysical foregrounds, particularly polarization by dust grains aligned by the galactic magnetic field and also synchrotron emission by relativistic electrons. The latter can in principle be accounted for through their angular power spectra and frequency dependence, distinct from that of the CMB.

The QUBIC Collaboration [11] reports the outcomes of end-to-end simulations of the QUBIC results from 2 years of operation of the full instrument, setting the input for primordial B-modes to $r=0$. There it is shown that using the spectro-imaging capabilities it is possible to reconstruct frequency sub-bands and obtain 5 maps that are used to estimate the parameter $r$ as well as three-parameters B-modes dust emission model (from latest Planck model). The width of the likelihood on $r$ shows a sensitivity $\sigma(r)=0.013$. Table 3.1 reproduced from [9], lists the expected sensitivity of QUBIC and other ground-based experiments in the same frequency range, either running or expected to be deployed in the near future. It is highlighted in 9 ] that not only the reachable sensitivity should be taken into account, but also the systematic effects control and the possibility to remove foregrounds. In this sense, QUBIC has a unique status due to its particular architecture as a bolometric interferometer.

Current LA involvement: QUBIC International Collaboration is integrated by France, Italy, UK, Ireland, USA and Argentina. In Argentina, 43 members (researchers, engineers, technicians and students) are part of different working groups. The involved institutions are CNEA (CAC, CAB, NOA and Cuyo), ITeDA (CNEA, CONICET, UNSAM) Instituto Balseiro and UNLP. As part of the local work $5 \mathrm{PhD}$ thesis are in progress. For the first module, Argentina is the only country in Latin America involved. In the future, for operation and installation of all the observatory, it is possible that new agreements with more countries in the region could be signed.

Local facilities and resources: Development of Alto Chorrillo site (a new scientific/astronomical pole in Salta, Argentina, where LLAMA will be also located). This site is $180 \mathrm{~km}$ away from the Chajnantor site in Chile where other millimeter-wave experiments and observatories are located (ALMA, APEX, Advanced 
Table 3.1: Sensitivity of the main B-mode ground experiments operating in a frequency range similar to QUBIC. The label "fg" or "no fg" corresponds to the assumption on the foregrounds. Taken from [9].

\begin{tabular}{lllll}
\hline Project & Frequencies $(\mathrm{GHz})$ & $\ell$ range & $\sigma(r)$ goal (no fg.) & $\sigma(r)$ goal (with fg.) \\
\hline QUBIC & 150,220 & $30-200$ & $6.0 \times 10^{-3}$ & $1.0 \times 10^{-2}$ \\
Bicep3/Keck & $95,150,220$ & $50-250$ & $2.5 \times 10^{-3}$ & $1.3 \times 10^{-2}$ \\
CLASS $*$ & $38,93,148,217$ & $2-100$ & $1.4 \times 10^{-3}$ & $3.0 \times 10^{-3}$ \\
SPT-3G $\dagger$ & $95,148,223$ & $50-3000$ & $1.7 \times 10^{-3}$ & $5.0 \times 10^{-3}$ \\
AdvACT $\ddagger$ & $90,150,230$ & $60-3000$ & $1.3 \times 10^{-3}$ & $4.0 \times 10^{-3}$ \\
Simons Array & $90,150,220$ & $30-3000$ & $1.6 \times 10^{-3}$ & $5.0 \times 10^{-3}$ \\
SO (SAT) ** & $27,39,93,145,225,280$ & $30-300$ & $1.3 \times 10^{-3}$ & $3.9 \times 10^{-3}$ \\
\hline ** CLASS: Cosm
\end{tabular}

**CLASS: Cosmology Large Angular Scale Surveyor; $\dagger$ SPT-3G: South Pole Telescope-3rd generation; $\ddagger$ AdvACT: Advanced Atacama Cosmology Telescope; ${ }^{* * S O}($ SAT) $=:$ Simons Observatory Small Aperture Telescopes.

ACTPol, POLARBEAR and CLASS) and offers similar atmospheric properties. This location has been characterized during several years within the framework of the site selection process for the CTA project, and for the LLAMA project, which has atmospheric requirements similar to those of QUBIC. The specific contributions from Argentina involve roads, energy, telecommunications, qualified human resources, contact with the community.

Scientific advances: The contributions of the argentine QUBIC collaboration can be grouped in simulations for the data acquisition, map-making process and component separation to clean the CMB and obtain forecasts on the parameter $r$. In particular, the study of the angular resolution of the reconstructed CMB maps obtained with the simulation pipeline for different sub-frequencies within the $150 \mathrm{GHz}$ band, taking into account the spectro-imaging capabilities of QUBIC [12]. Another important contribution is the improvement of the end-toend pipeline, and the simulations of the reconstructed maps, that are useful for the study of the instrumental noise. The development of a component separation framework to be able to separate the CMB signal from the foregrounds and studying the ability of QUBIC for distinguishing between different dust models in the data analysis procedure to obtain $r$ and the dust model parameters.

Technological advances: Several Argentine research laboratories have started to work on topics related to the instrument. In order to be able to provide detectors for future instruments, the development of new detector architectures with possibly reduced complexity but enhanced performance, has begun. Also, new readout electronics is being developed.

Advanced training: The collaboration is planning to develop a series of advanced schools for young scientists and engineers (see for example http://lapis2018.fcaglp.unlp.edu.ar/index.html). A program of grants to cover specific topics and allow the incorporation of new students in different stages of their careers is also part of the medium and long term educational purposes of the project.

Connection with industries: There is no direct involvement of local industry in the project. Nevertheless, for the fabrication of non-standard equipment and the QUBIC mount, a rotary mechanics with high precision requirements, private companies will be contracted.

Timeline and major milestones: The instrument is currently in laboratory calibration phase of its so-called "Technological Demonstrator". The next steps are to test and to install the first module in the site, with first light expected for 2021. The access road to the LLAMA site and from there to the QUBIC site has already been built, and construction for the installation of the first module is under way.

Computing requirements: For the data analysis, several $\mathrm{Tb}$ of storage will be needed, which will be provided at NERSC computing center, where the final analysis will be performed.

\subsubsection{South American Gravitational-Wave Observatory (SAGO)}

SAGO [13] is a proposed next generation detector for gravitational waves (GW). The emerging field of gravitational wave astronomy had its first experimental breakthroughs in the last five years, with the detection of mergers of binary black holes [14] and binary neutron stars [15] by a network of three advanced gravitational wave detectors: two in the United States (LIGO-Hanford, and LIGO-Livingston) and one in Europe (Virgo). These two mergers, are the most prominent amongst dozens of such events detected in three observing campaigns to date. Together, LIGO and Virgo observations have enabled various high-impact studies ranging from 
constraints on the equation of state of neutron stars, to tests of general relativity, and cosmological measurements. In the area of cosmology, in particular, advanced gravitational wave detectors have led to novel independent measurements of the present rate of cosmic expansion (the Hubble constant, $H_{0}$ ) [16-[18] which are crucial to shed light onto one of the greatest scientific problems of our time: the physical nature of the accelerated expansion of the universe. While the international GW community continues to upgrade and expand the existing network (e.g. adding a detector in Japan, KAGRA), they have also already started planning the next generation of detectors, known as the 3rd generation (3G) GW observatories. Two such detectors have been proposed: Cosmic Explorer (in the U.S.) and Einstein Telescope (in Europe). These new detectors will represent a giant leap in technology, enabling for the first time detection of mergers at high redshifts $(z \sim 30)$. The Latin American community is proposing to build its own 3G GW detector, SAGO. Such a detector will definitely put Latin America in a leadership position in the exciting new field of gravitational wave astronomy, with particular impact on multi-messenger cosmology.

Current and planned involvement: Latin America has active groups of scientists invested in both the LIGO and Virgo collaborations. The community seeks to grow in size, capability, and impact in the next decade aiming at the ambitious goal of becoming the hosts of a 3rd generation GW detector: SAGO.

Local facilities and resources: While the selection of the SAGO site will require an extensive technical study, a preliminary assessment performed by the USP-IAG seismic group identified three suitable locations: 1) near the Brazil Rio Grande do Sul and Uruguay border, 2) near Brazilian states of São Paulo and Mato Grosso do Sul, 3) "Triângulo das Secas" in the Brazilian northeastern region. Two other regions (not seismically calm) are also considered: 1) The Uyuni salt flat in Bolivia, and 2) The AUGER facility.

Scientific advances: The proposed $3 \mathrm{G}$ projects are complementary to each other: the larger the network, the better the results (including localization area, distance determination, and physical properties such as mass and spin). As part of the $3 \mathrm{G}$ network, SAGO will bring about a new cosmology breakthrough, enabling measurements of the cosmic expansion history since 100 Myrs after the Big Bang.

Technological advances: The community plans to focus on detector technology, learning from the experience with the current detectors and performing R\&D work towards the $3 G$ era. Advances in areas such as core optics, light sources, coatings, and cryogenics, are anticipated.

Advanced training: The Latin American GW community will grow by training PhDs locally (gaining experience through participation in current state of the art interferometers) as well as by attracting and retaining experts from abroad.

Connection with industry: Laser technology and optics are examples of areas of synergies with industry.

Timeline and major milestones: Over the coming decade, the priority is to build a critical mass of researchers with technical expertise. Goals are to perform R\&D and site determination studies. SAGO will then be built in the following decade, and join the 3G GW network in the 2040s.

Funding profile: SAGO is roughly estimated to be in the range of US\$ 1-2 Billion.

Computing requirements: Computing support is needed for: data processing, analysis, simulations.

\subsubsection{Vera Rubin Observatory's Legacy Survey of Space and Time (LSST)}

LSST is a survey of over 1 billion objects in the sky, using a wide field of view camera on a large aperture telescope in Chile. Among other goals, LSST will explore the nature of DM and DE. It will do so by analyzing over 20 terabytes of data per night to measure the distribution of galaxies, clusters of galaxies, and supernovae, as well as the distortions of the shape of galaxies produced by intervening matter. The community plans to implement an independent Data Access Center (iDAC) for LSST in Brazil [19].

Current LA involvement: The Brazilian participation in LSST is coordinated by the Laboratório Interinstitucional de e-Astronomia (LineA, linea.gov.br) and includes 9 Pls and 19 junior members.

Local facilities and resources: LIneA's computer facilities presently dedicated to LSST work consist of three clusters yielding a combined total of approximately 25 Tflops of computing power that will operate at $100 \mathrm{~Gb} / \mathrm{s}$. Moreover, the Laboratório Nacional de Computação Cientifica (LNCC) provides access to a supercomputer called Santos Dumont which has 1 PFLOPS available for shared use. It is expected that, by 2021, dedicated computing power will increase to over 100 Tflops with funds already allocated to LIneA by the federal funding agency FINEP.

Scientific advances: LSST will use measurements of the galaxy distribution, weak gravitational lensing, supernova and cluster counts to find the best fit cosmological model parameters with unprecedented accuracy 
and precision. In figure 3.2 it is shown the forecast on the parameters of the dark energy equation of state parametrized as $w(a)=w_{0}+w_{a}(1-a)$.

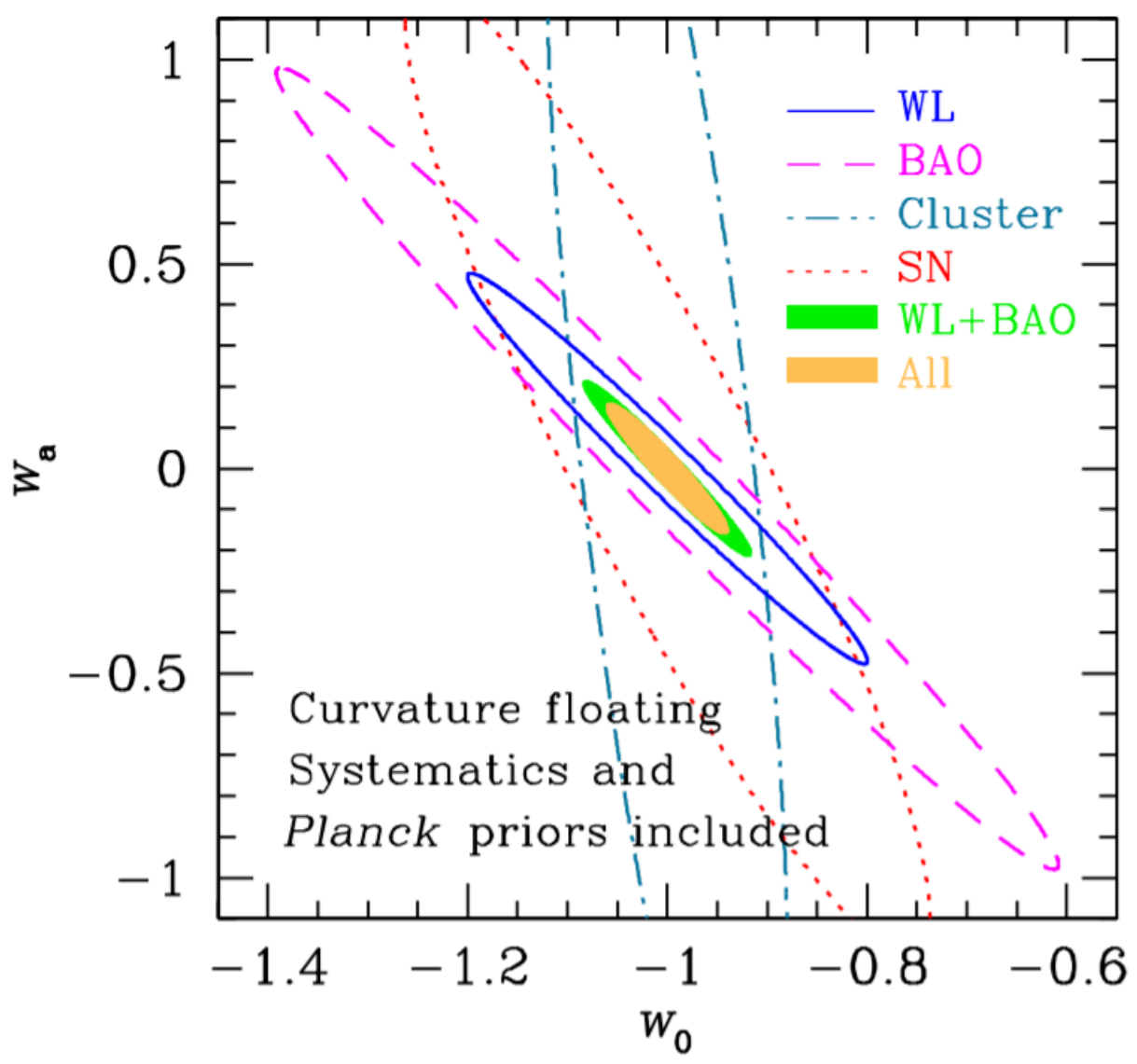

Figure 3.2: Forecast for the accuracy in the estimation of Dark Energy parameters $w_{0}$ and $w_{a}$ from multiple probes at the LSST. Figure reproduced from the LSST Dark Energy Science Collaboration (DESC) Science Requirements Document.

Technological advances: Rapid image processing and advanced machine learning techniques are among the core developments of LSST.

Advanced training: LSST research groups in Brazil will produce PhDs with expertise in state of the art cosmic survey analyses.

Connection with industry: Synergistic opportunities involving computational methods include machine learning and ultra-fast image processing.

Timeline: LSST is scheduled to start observations in 2022, lasting until at least 2032.

Computing requirements: Massive computational resources are necessary for photometric redshift estimation of a billion galaxies, production of galaxy catalogs, weak lensing catalogs, cluster catalogs, supernova catalogs and for running the analysis pipelines.

\subsubsection{Latin American PhD program}

The goal is to create a collaborative LA PhD program in Astrophysics, Cosmology and Gravitation, as a network of institutions from different countries in the continent. Progress in this field with global impact not only needs research facilities and possibilities to access observational data, but also qualified human resources, a very important part of research infrastructures. The proposal described in [20] is based on the PPGCosmo experience (which is a Brazilian PhD program based at UFES) and on the observation that participation of many LA countries in astronomical facilities installed in LA is below what could be expected. To create the 
program, firstly, it is important to form an advisory scientific committee composed of leading scientists from different countries working on those subjects. The institutions and research groups must express explicitly the interest to join the proposed program, with the necessary agreement terms. Hence, the necessary scientific and administrative personnel, as well as the infrastructure, are in principle already available. The important new contributions to this project would be a significant number of PhD fellowships, which should be provided by the different countries, together with a specific financial support for scientific missions within the continent. It would be important that those fellowships have a fixed common value to all students. The main goal is to carry out a common $\mathrm{PhD}$ formation, inducing a deep scientific collaboration among all participating institutions and research groups with extensive use of the astronomical facilities existing in the continent. The PhD student will develop their project thesis under the supervision of at least two advisors from at least two institutions in different countries. In order to simplify the bureaucratic work the PhD certificates would be emitted by one host institution and immediately recognized and validated by all institutions joining the project. Since the student internship period in at least two institutes would be a mandatory requirement to obtain the PhD degree this will induce a closer interaction among the institutions and research groups participating in the program.

\subsection{Areas of Excellence}

Latin America is home to a vibrant research community in cosmology. With strong participation in projects ranging from cosmic surveys [11, 19] to gravitational wave detectors [13], Latin American groups contribute to cutting-edge results in the field. Remarkable success has been achieved despite their small size relative to groups in U.S. and Europe.

For example, in the recently concluded Dark Energy Survey (DES, [21]), the LineA team (in Brazil) has led data processing pipelines and important analysis developments, taking leadership roles within the collaboration and producing high-impact publications. The team has grown to include dozens of researchers many of whom now form the core of the Brazilian participation in the upcoming LSST project. Similarly, the experience of Latin American researchers in LIGO and Virgo has seeded a community that is now ready to take a bold step towards a leadership role in the next generation of GW experiments.

Specific areas of excellence include: 1) high performance computing and instrumentation for cosmic surveys and telescope facilities operating in wavelengths ranging from optical to sub-millimeter regimes; 2) detector development, operations, and data analysis for GW observatories.

Despite challenges regarding sustained funding for long term projects, the community has successfully leveraged its resources and has demonstrated ability to take leadership roles in larger scientific endeavors in the coming decades.

\subsection{Synergies}

In synergy with QUBIC CMB polarization measurements at large-scale angular detection (degree), a bolometer has begun to be designed to be placed in the LLAMA Cassegrain cabin to measure polarization on smaller scales (arcmin or smaller) for the same part of the sky. The experiment would complement QUBIC search for primordial B-modes, by measuring the secondary B-modes. In addition, it could also have an impact on other scientific goals, such as testing the main pillars of the base cosmological model, the properties of the dark sector, the sum of neutrino masses.

In synergy with neutrino experiments, cosmology can probe the sum of the neutrino masses. In the based $\Lambda$ CDM model with three massive neutrinos, the current limits obtained from CMB and BAO data, is $m_{v}=$ $\sum_{i} m_{v_{i}}<0.12 \mathrm{eV}(95 \% \mathrm{CL})[10]$. This bound is close to the minimum allowed by the inverted hierarchy. If the mean value of $m_{V}$ is sufficiently less than $0.1 \mathrm{eV}$ (but not if $m_{V}>0.1 \mathrm{eV}$ [22]), which can be the case only if the neutrino masses follow the normal hierarchy, results from future surveys such as EUCLID are expected to be able to differentiate between the two hierarchies [23]. The sensitivity of Planck+Euclid to a neutrino mass sum of $0.06 \mathrm{eV}$ (close to the minimum level allowed by oscillations) is $\sigma\left(m_{v}\right)=0.02 \mathrm{eV}[23]$.

Studies of models of DM, DE, and gravity are extremely complementary, and the understanding of one of them cannot be achieved without the understanding of the others. Astrophysics and cosmology offer a complementary approach to study the fundamental properties of DM. They probe DM directly through gravity, the only force to which DM is known to couple. In synergy with direct and indirect DM detection experiments, LSST is expected to provide complementary tests of well-motivated theoretical models of DM (such as warm 
DM, self-interacting DM, DM- baryon interactions, axion-like particles, ultra-light DM, and primordial BHs) and help to constrain DM-baryon scattering, DM self-annihilation, and DM decay. GW experiments can also test some DM models (such as ultra-light DM and primordial BHs candidates).

In addition, CTA [24] will also be able to measure the interaction of Very High Enery (VHE) photons with the Extragalactic Background Light (EBL). The EBL is difficult to measure and detect, due to its intrinsic faint surface brightness and strong contamination by foreground sources, but provides an important tool to measure Cosmic Voids and the overall baryonic matter distribution in the Universe, providing important cosmological constraints. The imprint of this structure on the VHE photons, however, will be detectable by CTA, offering a new way to measure the evolution of cosmic voids with cosmological time.

Likewise, GRAND [25] is expected to provide important information on the birth of galaxies in the dark ages, with profound cosmological impact. By operating at radio $\mathrm{MHz}$ frequencies, GRAND will be able to detect the absorption from the hyperfine transition of the hydrogen atom at very high redshifts $(z \sim 20)$, following on the first tentative detection of the signal from the Epoch of Reionization (EoR) by EDGES [26].

Finally, one should emphasize the many synergies of cosmological studies with the evolution of galaxies, as the building blocks of the LSS in the universe. These areas are inherently complementary, and as such could benefit strongly from interactions and a joint observational structure - with the Vera Rubin Observatory being a prime example of complementary goals achievable by the same observations.

\subsection{Conclusions}

While the LA cosmology community remains rather sparse and scattered about the continent, significant progress has been made towards greater coordination among groups. Leveraging local facilities and resources, individual Latin American groups that have traditionally grown in isolation are now pursuing or considering joint ventures. The current landscape of projects is balanced between Latin American led initiatives and global collaborations with Latin American participation. Proposed Latin American led experiments include BINGO and SAGO. Global experiments located in Latin America include QUBIC, TOROS and ABRAS, and LSST. This involves the development of the new astronomical sites at the Macon Ridge (home to the Toros and ABRAS experiments) and Alto Chorrillos (where QUBIC and LLAMA are located) which are both in the northwest corner of Argentina, in the Atacama Plateau shared with Chile.

Apart from these notable examples which involve part of the Chilean, Brazilian and Argentinean communities, most of the LA community members are currently dedicated to theoretical aspects not directly connected with any experimental facility. Such theoretical studies continue to be essential to exploit observational data and experimental results, deepening the understanding of their connection with fundamental physics and helping to motivate new ways to explore nature.

However, the LA community faces several challenges when addressing its main science drivers. One problem is the lack of long term stability of funding and variations in the landscape from country to country. This discourages large scale joint projects despite the fact that know-how and critical mass have already been achieved.

The white papers indicate broad community agreement on the benefits of joining forces between different groups and countries despite these challenges. Capacity building and collaborative work are important elements. LA-CoNGA [27] Physics project and the proposal of the Latin American PhD program (which emphasizes the need of increasing Latin American participation in facilities installed in the region and involves diverse topics), are clear examples highlighting these key elements. The need to participate in international collaborations has also been emphasized. 


\section{Bibliography}

[1] Oliver H.E. Philcox, Mikhail M. Ivanov, Marko Simonović, and Matias Zaldarriaga. Combining Full-Shape and BAO Analyses of Galaxy Power Spectra: A 1.6\% CMB-independent constraint on $\mathrm{H}_{0}$. JCAP, 05:032, 2020.

[2] L. Verde, T. Treu, and A.G. Riess . Tensions between the Early and the Late Universe. 72019.

[3] J. S. Bullock and M. Boylan-Kolchin. Small-Scale Challenges to the $\Lambda$ CDM Paradigm. Astron. Astrophys. , 55:343, 2017.

[4] Abbott, B. P., Abbott, R., Abbott, T. D., et al. . Multi-messenger Observations of a Binary Neutron Star Merger. ApJ, 848:L12, 2020.

[5] Ellis, Richard Keith and others. Physics Briefing Book: Input for the European Strategy for Particle Physics Update 2020. 102019.

[6] Díaz, M. C., Macri, L. M., Garcia Lambas, D., et al. Observations of the First Electromagnetic Counterpart to a Gravitational-wave Source by the TOROS Collaboration. ApJ 848 (2), page L29, 2017.

[7] et. al. Cowperthwaite, P. S.

[8] Mario Díaz, Diego Garcia Lambas, Omar Lopez Cruz Lucas Macri, Claudia Mendes de Oliveira, and José Luis Nilo Castellón. Macón Ridge Astronomical Site The ABRAS and TOROS projects. White Paper submitted to the Latin American Strategy Forum for Research Infrastructure (LASF4RI), 2020.

[9] Claudia G. Scóccola, Beatriz García, Diego Harari, Gustavo Romero, Alberto Etchegoyen. QUBIC: Q\&U Bolometric Interferometer for Cosmology. White Paper submitted to the Latin American Strategy Forum for Research Infrastructure (LASF4RI), 2020.

[10] Planck Collaboration: N. Aghanim, Y. Akrami, M. Ashdown, J. Aumont et al. Planck 2018 results. VI. Cosmological parameters. arXiv eprints 1807.06209, 2018.

[11] P. de Bernardis et al. (QUBIC Collaboration). QUBIC: Measuring CMB polarization from Argentina. BAAA 60, page 107, 2018.

[12] Gamboa-Lerena et al. BAAA 62, in press, 2020.

[13] O. Aguiar and M. E. S. Alves and C. F. S. Costa and C. H. Lenzi and R. Sturani (SAGO Collaboration). The South American Gravitational wave Observatory (SAGO). White Paper submitted to the Latin American Strategy Forum for Research Infrastructure (LASF4RI), 2020.

[14] B. P. Abbott et al. (LIGO \& Virgo Collaborations). Observation of Gravitational Waves from a Binary Black Hole Merger. Phys. Rev. Lett., 116:061102, 2016.

[15] B. P. Abbott et al. (LIGO \& Virgo Collaborations). GW170817: Observation of Gravitational Waves from a Binary Neutron Star Inspiral. Phys. Rev. Lett., 119:161101, 2017.

[16] The LIGO Scientific Collaboration, The Virgo Collaboration, et al. A gravitational-wave standard siren measurement of the Hubble constant. Nature, 551:85, 2017.

[17] M. Soares-Santos, A. Palmese, and W. Hartley et al. (DES, LIGO \& Virgo Collaborations). First Measurement of the Hubble Constant from a Dark Standard Siren using the Dark Energy Survey Galaxies and the LIGO/Virgo Binary-Black-hole Merger GW170814. Astrophys. J. Lett., 876:L7, 2019.

[18] A. Palmese, J. deVicente, and M. E. S. Pereira et al. (DES Collaboration). A statistical standard siren measurement of the Hubble constant from the LIGO/Virgo gravitational wave compact object merger GW190814 and Dark Energy Survey galaxies. Astrophys. J. Lett. (accepted), arXiv:2006.14961, 2020.

[19] R. Rosenfeld. Cosmology with the Large Synoptic Survey Telescope. White Paper submitted to the Latin American Strategy Forum for Research Infrastructure (LASF4RI), 2020.

[20] Hermano Velten and Júlio Fabris. A Latin American graduate school - The PPGCosmo experience. White Paper submitted to the Latin American Strategy Forum for Research Infrastructure (LASF4RI), 2020. 
[21] T. Abbott et al. (DES Collaboration). The Dark Energy Survey: more than dark energy - an overview. Mon. Not. R. Astron. Soc., 460(2):1270, 2016.

[22] Shouvik Roy Choudhury and Steen Hannestad. Updated results on neutrino mass and mass hierarchy from cosmology with Planck 2018 likelihoods.

[23] Tim Sprenger, Maria Archidiacono, Thejs Brinckmann, Sébastien Clesse, and Julian Lesgourgues. Cosmology in the era of Euclid and the Square Kilometre Array. JCAP, 02:047, 2019.

[24] Elisabete M. de Gouveia Dal Pino,Ulisses Barres de Almeida et. al. WHITE PAPER - High Energy Astrophysics and Cosmology The Cherenkov Telescope Array (CTA). White Paper submitted to the Latin American Strategy Forum for Research Infrastructure (LASF4RI), 2020.

[25] Rafael Alves Batista et. al. . GRAND: Giant Radio Array for Neutrino Detection. White Paper submitted to the Latin American Strategy Forum for Research Infrastructure (LASF4RI), 2020.

[26] Bowman, J. D., Rogers, A. E. E., Monsalve, R. A., Mozdzen, T. J. \& Mahesh, N. An absorption profile centred at 78 megahertz in the sky-averaged spectrum. Nature 555, page 67, 2018.

[27] José Ocariz, et. al. LA-CoNGA Physics perspectives for the Latin America Strategy Forum for Research Infrastructure (v1.0). White Paper submitted to the Latin American Strategy Forum for Research Infrastructure (LASF4RI), 2020. 


\section{Dark Matter}

Marcela Carena (Fermilab, US)

Diego Restrepd ${ }^{1}$ (Instituto de Física, Universidad de Antioquia, Colombia)

\subsection{Introduction}

This chapter focuses on activities in the Latin American region, for a complementary worldwide range of activities see the recent European Strategy update [1].

One of the questions for fundamental science which clearly points to the existence of Beyond Standard Model physics is Dark Matter (DM), an unknown, non-baryonic matter component, whose abundance in the Universe exceeds the amount of ordinary matter roughly by a factor of five. The evidence for DM stems from different times and distance scales of our Universe. The precise measurements of the cosmic microwave background (CMB) power spectrum, galaxy rotation curves, galaxy clusters, gravitational lensing, baryonic acoustic oscillations and Big Bang Nucleosynthesis (BBN) have shaped our understanding of the universe and represent a whole panoply of evidence for DM. However, all these data points to DM only through it gravitational interactions, giving no specific information about its nature. Despite the fact that DM has been searched for decades in terrestrial and extraterrestrial experiments and probes, those searches have given no specific information of what DM actually is.

There is lack of information on how DM was produced in the early universe, before BBN, and therefore, the expected DM signals of the relic density observed today depend significantly on the specific assumptions of its unknown history. Supposing that the same standard cosmology holds before BBN, the simplest hypothesis is to assume that the DM was thermally produced along with the other SM particles. Then, by considering a weak-like interaction strength of the DM with SM particles, the relic density can be easily accounted for. This is the so call Weakly Interacting Massive Particle (WIMP) miracle. In the previous decades, the WIMP mechanism scenario has received by far the biggest attention, both theoretically and experimentally. WIMPs typically carry electroweak scale mass and couple to the SM with a strength that is reminiscent to that of the weak interactions. Theories beyond the SM (BSM), such as supersymmetry, naturally provide excellent DM candidates (e.g. electroweakinos) that are among the most studied [2-6].

The non-observation of WIMP-like DM, however, puts this mechanism under scrutiny, both at experimental and theoretical levels. Moreover, as the WIMP paradigm becomes constrained, there is nowadays no single compelling theoretical framework for DM, but it rather opens a wealth of cosmological possibilities. Despite our ignorance concerning the DM particle, we have accumulated important information on the basic requirements for a DM candidate: (i) it should yield the correct relic density; (ii) it should be non-relativistic at the epoch of matter-radiation equality, to form structures in the early Universe in agreement with observations; (iii) it should be effectively neutral, otherwise it would have formed unobserved stable charged particles; and (iv) it should be cosmologically stable, with a lifetime much larger than the age of the universe, and consistent with cosmic rays and gamma-rays observations. Having these requirements in place, we delineate four classes of DM candidates to be considered:

- WIMPs: They appear in several, well motivated model building setups. They are based on the thermal decoupling paradigm which is a key input in observables such as the CMB and BBN. Under these assumptions, the correct relic density is obtained for an annihilation cross-section of the order of the electroweak scale and DM particles with roughly weak scale $\mathscr{O}(100) \mathrm{GeV}$ masses. Typically the parameters that govern the relic density are deeply intertwined with those in the direct and indirect detection signatures as well as with collider searches [7-13]. Many WIMP models can reproduce the correct relic density and

\footnotetext{
$\sqrt[1]{\text { restrepo@udea.edu.co }}$
} 
yield signals that are within reach of current and upcoming experiments [14], with prospects of leading to a very rich experimental program.

- Hidden Sector DM, including Light DM: As the WIMP paradigm is being challenged, models of Hidden Sector DM, that provide thermal relics in the mass range between about a few keV to $100 \mathrm{TeV}$ and may interact with laboratory detectors via new exotic, extremely weak interactions are under high scrutiny. In particular, the keV-GeV mass range, also referred as Light DM (LDM) demands new technologies, new detection strategies and more sophisticated analyses that typically fail above the GeV region. In particular, in the LDM region the direct detection searches become powerful through the exploration of DM-electron interactions. Moreover, in order to reproduce the observed DM relic density, models with LDM typically require the existence of light mediators that themselves can be searched for at laboratory experiments.

- Ultralight DM (ULDM), including Axions and Axion-like Particles (ALPS): It is possible to have nonthermal ULDM candidates whose masses expand the range $10^{-22} \mathrm{eV} \lesssim m_{\Phi} \lesssim 1 \mathrm{eV}$, with extraordinary weak couplings to SM fields. Such ULDM (lighter than about $0.7 \mathrm{keV}$ ) must be bosonic [15], and the effects of the halo DM in terrestrial experiments are best described as wavelike disturbances. Axions are well-motivated DM candidates originally thought of as an outcome of the Peccei-Quinn (PQ) solution of the strong CP problem [16. 17]. The axion is the pseudo-Nambu-Goldstone boson of the U(1) $P Q$ symmetry [18, 19], which is spontaneously broken at an energy scale related to the axion mass and couplings. The axion DM mass range is $10^{-12} \mathrm{eV} \lesssim m_{a} \lesssim 10^{-2} \mathrm{eV}$. More general ALPS - with a more flexible range of axion mass and couplings - are also possible, allowing for axions and ALPS in the sub$\mathrm{eV}$ region to be stable at cosmological mass scales, and provide suitable DM candidates. Many BSM scenarios, some inspired by string theory, contain ALPS and other sub-eV dark sector particles such as dark scalars or dark photons that can provide non-thermal DM candidates. A new set of table top experiments with novel technologies are being explored worldwide [20].

- Non-thermal or quasi-thermal DM; There is a broad class of DM scenarios with masses above the ULDM range but that are also produced non-thermally. The DM masses can span from the keV to $100 \mathrm{TeV}$, with interaction strengths varying quite vastly. Examples of such models include the Strongly Interacting Massive Particle (SIMP), the asymmetric DM (ADM), the Feebly Interacting Massive Particle (FIMP), etc. It is important to keep an open mind towards non-thermal histories that can still fulfill the aforementioned requirements and therefore constitute viable DM candidates.

\subsection{Astrophysical and cosmological probes of DM}

Dark Matter - if made of particles and compact objects - is the dominant form of matter in the universe, and, hence, there are several experiments that try to measure its interactions with detector materials, as well as the results of annihilation from regions in which the density is expected to be sufficiently high. There is a large array of experiments exploring the DM paradigm through terrestrial and extraterrestrial probes. In this section we shall only mention the ones with active Latin American involvement.

\subsubsection{Direct detection}

Direct detection refers to the observation of nuclear recoils at low energy experiments located at deep underground laboratories. By measuring the energy recoil and the shape of the scattering rate of the events, one may reconstruct the DM-nucleon (or DM-electron) scattering cross-sections and infer the DM mass. Using different targets and readout techniques several experiments have been proposed.

DarkSide [21] is one of the leading experiments currently searching for WIMP-like DM and LDM candidates, having excellent opportunities for future term projections. The experiment is based on Liquid Argon (LAr) technology and efficiently searches for DM using scintillation and ionization readout techniques [22]. After a DM-Argon nucleus scattering, a prompt scintillation light is emitted (S1 signal), and an ionization process occurs at the same time. In the latter event, electrons are drifted towards the anode of the time projection chamber finding a gas phase of argon where they emit electroluminescent light (S2 signal) [23]. Both signal measurements are used in constraining the DM-nucleon scattering cross sections. It is notable that, just by taking advantage of the high trigger efficiency of the S2 signal events for low energy recoils [24] experimenters constrain DM-electron scattering and produce competitive bounds in the $20 \mathrm{MeV}-1 \mathrm{GeV}$ DM mass window. DarkSide current results are based on $50 \mathrm{Kg}$ of LAr, and the collaboration is now commissioning a 20 tonnes 


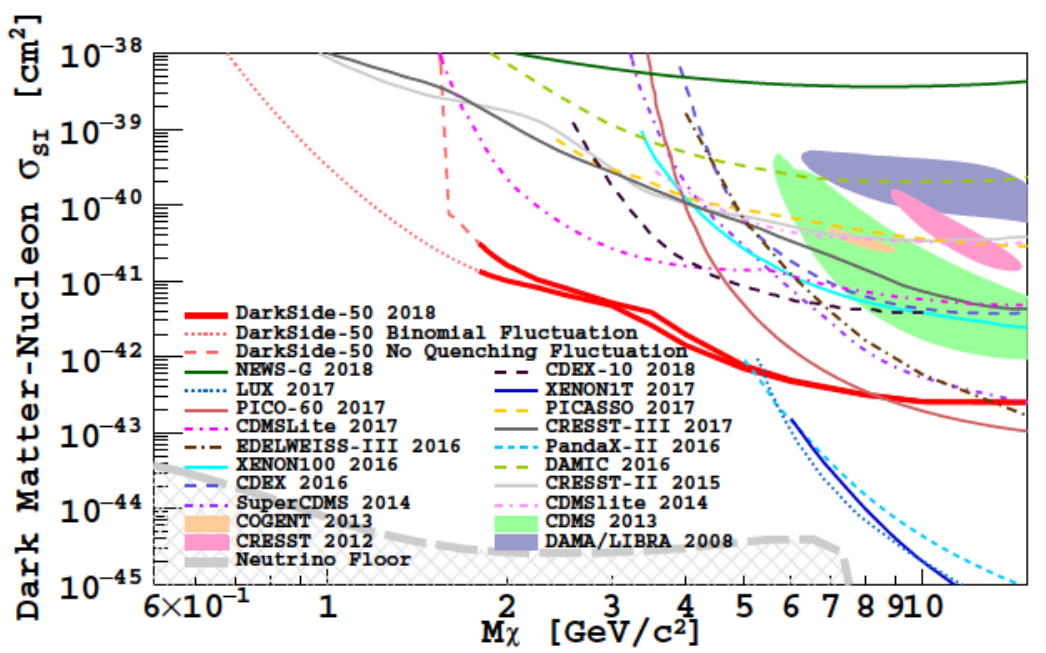

Figure 4.1: Upper limits on spin independent DMnucleon cross sections from DarkSide-50 in the range above $1.8 \mathrm{GeV} / \mathrm{c}^{2}$, from 23

LAr detector. Brazil is participating in this experiment through the group of Professor Ivone Albuquerque of University of Sao Paulo (USP), and Mexico is contributing through Professor Eric Vázquez Jáuregui of Universidad Autonoma de México (UNAM).

The DarkSide experiment currently has the best limits for DM-nucleon cross-section in the low mass region, DM masses in the 2-5 GeV range, as illustrated in Fig. 4.1 New phase DarkSide-20k will feature 20 tonnes of LAr with a future reach very close to the neutrino floor as illustrated in Fig. 4.2. While other experiments such as XENONnT and LZ will be highly competitive in the region of $10 \mathrm{GeV}$ masses and above, DarkSide seems to have a leading edge in the low energy region of 1-10 GeV. Future experiments like the Global Argon DM Collaboration (GADMC) are designed to reach the neutrino floor over a broad range of DM masses in the 100 $\mathrm{GeV}$ to $100 \mathrm{TeV}$ range. [25].

The LA region also has a very strong presence in the SENSEI [27] and future DAMIC-M [28] experiments, that have a unique opportunity to explore the light DM window through DM-electron cross-section measurements. The participation in SENSEl is through the Universidad de Buenos Aires (UBA), the Instituto de Fisica de Buenos Aires (IFIBA) and the Centro Atómico de Bariloche (CAB) in Argentina, while DAMIC-M is contributing through $\mathrm{CAB}$, Argentina. Both experiments use Skipper-CCD technology optimized to detect electronic recoils, a technology that has been developed with relevant involvement of Latin American scientists. This line of research is done in close collaboration with Fermi National Accelerator Laboratory (Fermilab) in the U.S. Skipper-CCDs are silicon pixelated detectors with a spatial resolution of $15 \mu \mathrm{m}$ and the ability to measure the deposited charge per pixel with a readout noise Root Mean Squared (RMS) of $0.1 e$. This unprecedented low noise level permits to count individual electrons, which translates to the lowest possible threshold detection energy. Thanks to its low readout noise level, Skipper-CCD technology is particularly competitive to test theories that predict LDM in the sub-GeV range.

SENSEI has the current best limit for LDM-electron scattering cross-section, $\bar{\sigma}_{e}$, with form factor $F_{\mathrm{DM}}(q)=$ $\left(\alpha m_{e} / q\right)^{2}$, which is illustrated in Fig. 4.3-left, and a competitive limit in LDM-nucleus scattering cross-section, $\bar{\sigma}_{n}$ for a light mediator, which is illustrated in Fig. 4.3 right. These are searches for direct observation of DM in the low mass domain, from the $\mathrm{MeV}$ range up to tens of $\mathrm{GeV}$. In these type of experiments, special care is needed to shield the detector from ambient radiation, be it unstable isotopes or cosmic showers, requiring the installation of the experiment in underground caverns. In this sense, SENSEl-100-gr-yr (next generation) will upgrade from its current location in the SOUDAN mine by moving to SNOLAB. A similar experiment, DAMIC-M is planned in Modane in the near future. In addition, already in the plans is the next generation of Skipper-CCDs detector based DM searches at the OSCURA experiment at SNOLAB, where two orders of magnitude gain in sensitivity with respect to SENSEI-100-gr-yr is expected. It is of great relevance to note that the prospective, $1700 \mathrm{~m}$ underground ANDES laboratory, to be constructed in parallel with the Agua Negra tunnel between Argentina and Chile, will provide a unique auspicious location for future generations of 


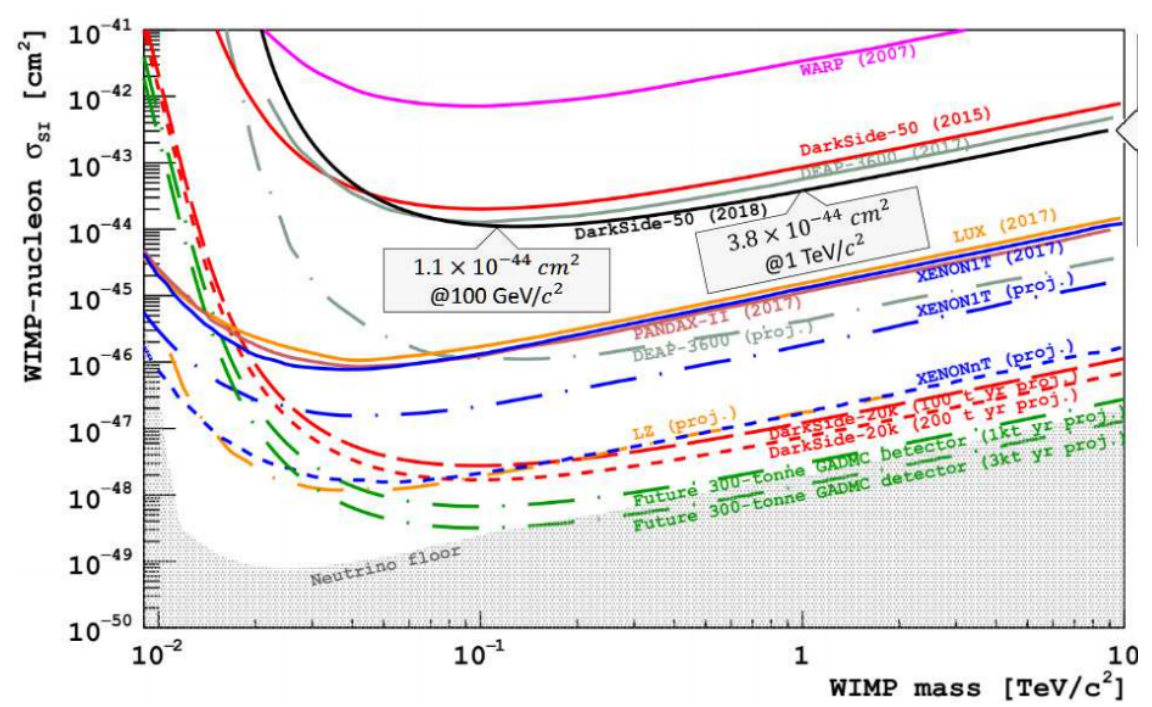

Figure 4.2: Spin-independent DM-nucleon cross section $90 \%$ C.L. exclusion limits from the analysis detailed in [26] (in black), compared with selected results and projections for XenoNnT (dash-blue) LZ (dash-orange) DarkSide-20k (dash-red) and the Global Argon DM Collaboration (GADMC) (dash-green), reaching the socalled neutrino floor [25].

Skipper-CCD based DM experiments in the southern hemisphere.
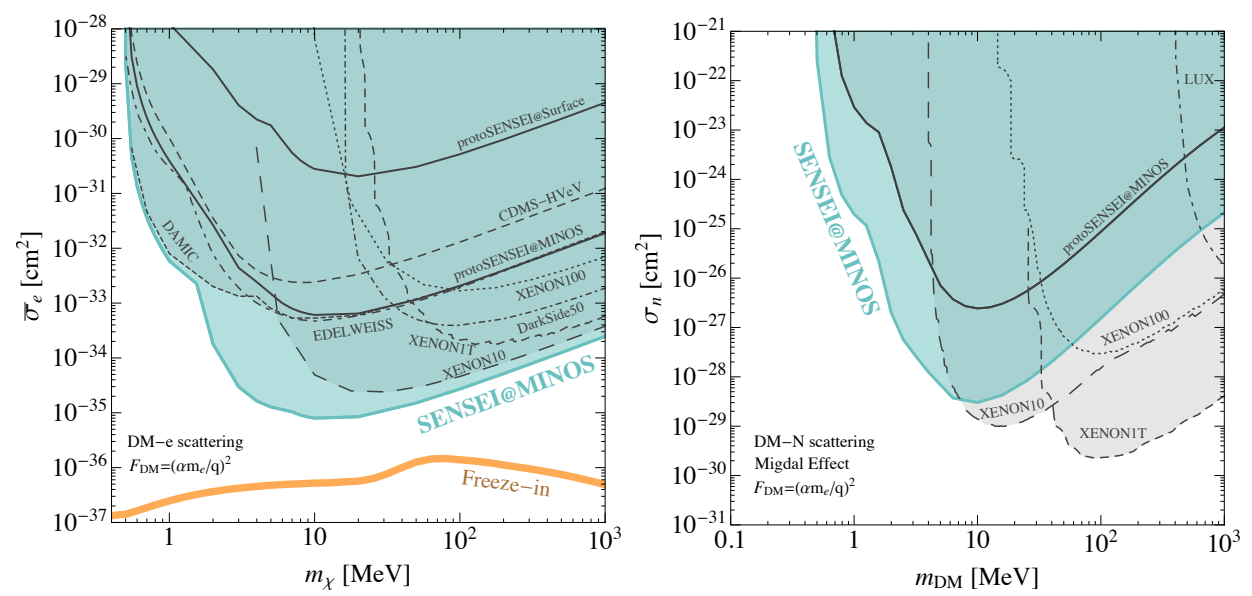

Figure 4.3: DM limits from SENSEI from [27], for DM-electron scattering cross-section and DM-nucleus scattering cross-section as a function of the DM mass. See text for details.

\subsubsection{Indirect detection}

Indirect DM detection refers to the observation of stable particle fluxes such as electrons, protons, neutrinos and gamma-rays produced by DM annihilation or decay in dense astrophysical environments, such as Dwarf Spheroidal Galaxies and the galactic center.

Chile and Brazil participate in the Cherenkov Telescope Array (CTA), while Argentina, Brazil, Mexico and Peru are part of the Southern Wide-field Gamma-ray Observatory (SWGO). Both experiments are described in the Astrophysics chapter of this report. In combination, both experiments will cover a wide range of heavy DM masses annihilating to bottom quark pairs, illustrated in Fig. 4.4 [29].

Hence, both CTA and SWGO will give important information about models of DM above the $100 \mathrm{GeV}$ mass range and in the absences of a signal will interestingly constrain heavy WIMP scenarios. In particular, for the large mass solution of the Inert Doublet Model (IDM) with DM masses in the range 0.5-3 TeV, one can 


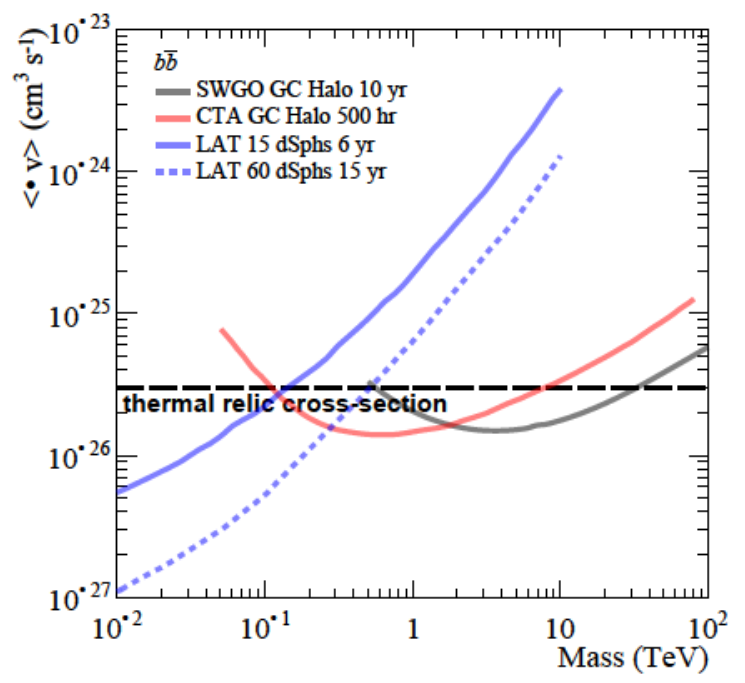

Figure 4.4: Expected 95\% C.L upper limit on the velocity-weighted cross section for DM self-anniliation into $b \bar{b}$ as a function of DM for CTA and SWGO observation of the galactic center halo 29.

conclusively say that CTA will exhaustively probe the IDM at the TeV scale [30].

\subsection{DM production at colliders}

The nature of DM and its interactions can be probed at accelerator based experiments, complementing experiments and observations from astroparticle physics and cosmology. The prospect of creating DM in the laboratory, either produced directly in beam-beam collisions with other SM particles or in decays of SM or yet to be discovered new ones is tantalizing. In all cases, the dark matter signal will consist of significant transverse missing energy plus high energetic SM objects, or complex SM final states in the case of cascade decays. There is a significant number of articles related to DM models, that have been published with strong involvement and leadership of Latin American scientists working on analysis techniques, and strategy searches at the LHC. For example, novel ideas and models to explore the production of anapole DM [31], DM in supersymmetric scenarios [4 32,33], models where lepto-quarks couple to dark matter [34], among others have been conducted. Additionally, new analysis techniques using vector boson fusion and initial state radiation jets [35], have been proposed using simulated data. Several of these searches have lately been investigated by the ATLAS, CMS and $\mathrm{LHCb}$ collaborations, all of them with participation from Latin American groups. We next illustrate the case for DM searches with the Compact Muon Solenoid (CMS) experiment.

The CMS detector at the LHC has a broad physics program to search for physics beyond the SM, including DM. The effort of several experimental groups of the Latin American community, in particular scientists at the Universidad de los Andes and Universidad de Antioquia, in Colombia, are working on DM searches using supersymmetric models and compressed mass spectra scenarios with tau leptons. The searches are carried out using two different techniques, vector boson fusion [36-38] and jets from initial state radiation [39]. These efforts have led to many publications that have facilitated the involvement of Latin American students and young scientists in the searches for DM and dark sectors at the LHC.

\subsection{DM portals}

As the WIMP paradigm becomes experimentally scrutinized with no evident positive results, extended scenarios with Hidden Sector DM and DM mediators (additional dark sector particles) become of interest. Dark matter mediators from the dark sector can then interact with SM or even BSM portals, including scalar/pseudoscalar, vector and fermion portals. In particular, it is promising to search for DM mediators decaying to SM particles or into the DM particles in a broad array of possibilities, from accelerator based experiments at colliders and fixed target probes (see discussion of DM/Dark Sector chapter in Ref [1] ) as well as through their possible interactions in reactor neutrinos experiments. 

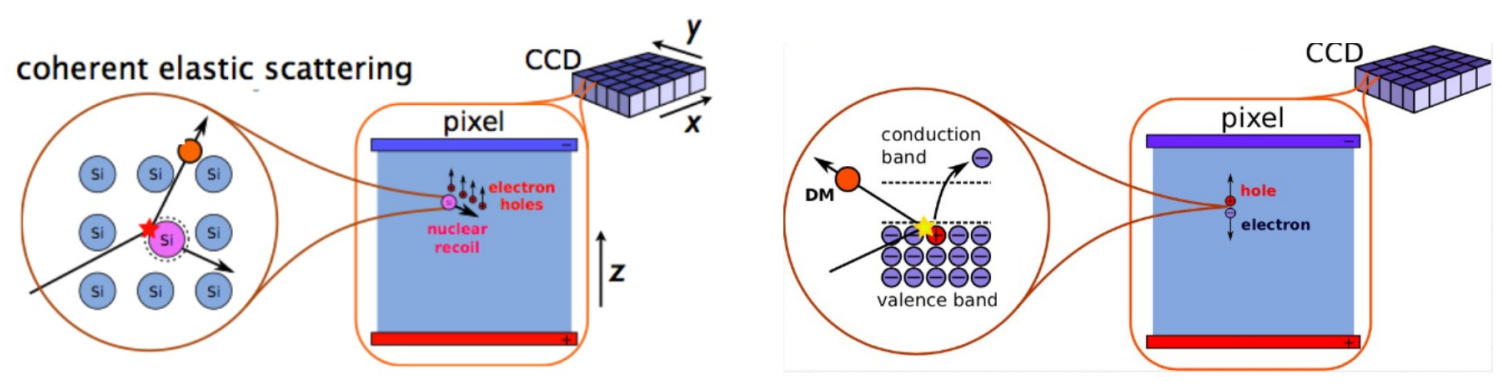

CONNIE experiment. I. Nasteva

Figure 4.5: CCD detectors in reactor and direct detection experiments.

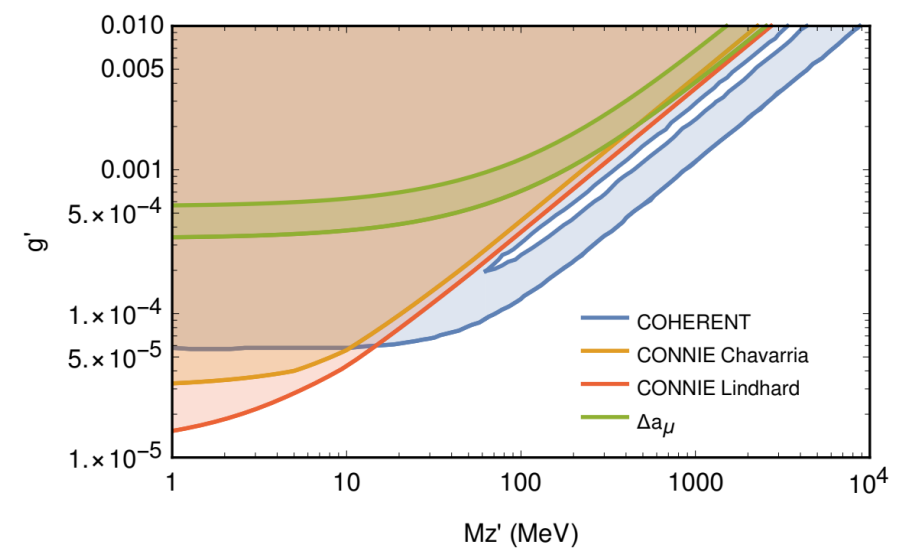

Figure 4.6: Exclusion region in the $\left(M_{Z^{\prime}}, g^{\prime}\right)$ plane from CONNIE results in [41].

The particle detection technology employed in SENSEI is directly applicable to the study of neutrino physics exploiting coherent neutrino-nucleus interactions, recently observed by the COHERENT Collaboration [40], as illustrated in Fig. 4.5. The Skipper-CCD detectors may prove to be sensitive to the low energy threshold of the coherent neutrino-nucleus interaction and can be used to measure the high intensity low energy neutrinos escaping from a nuclear reactor. By placing these detectors at different distances close to the reactor core one could search for new particles, such as $Z^{\prime}$ s ( that appear in some DM models), that could contribute to the coherent neutrino-nucleus interactions.

The current Coherent Neutrino-Nucleus Interaction Experiment (CONNIE), plans to upgrade to Skipper-CCD detectors as a step in the search for coherent elastic scattering processes mediated by BSM Z' interactions; see first limits for such a BSM portal in the current CONNIE experiment in Fig. 4.6 [41]. Similarly, vIOLETA ${ }^{2}$ is a future experiment with very similar goals as those discussed for CONNIE, based on Skipper-CCD technology and with the advantage of more flexibility towards its location vis a vis the neutrino reactor core. Two positions were identified to place vIOLETA within the Atucha II Nuclear Power Plant in Argentina, at 8 and 12 meters away from the center of the reactor core.

The Latin American region is building the Lambda laboratory 3 at the Physics Department at UBA, that is set to exploit Latin America's privileged position in Skipper-CCD technology. This specialized lab will permit to develop experimental collaborations for forefront DM searches and neutrino physics experiments. By the end of 2020 Lambda lab expects to have a first working setup, including a $50 \mathrm{~m}^{2}$ laboratory with turbo-molecular vacuum stations, cryo-refrigerators, low-radioactivity vacuum chambers and ultra low noise readout electronics for CCDs. The Lambda lab is working in close collaboration with the Skipper-CCD group at Fermilab.

There is a large participation of the Latin American region in the neutrino experimental program at Fermilab, including the future flagship experiment: the Deep Underground Neutrino Experiment (DUNE). This includes leading institutions in Brazil, Colombia, Mexico, Paraguay, and Peru. The DUNE experiment is based on liquid

\footnotetext{
${ }^{2}$ See https://www.violetaexperiment.com/

3 http://lambda.df.uba.ar/portfolio/
} 
argon detectors of mega-proportions exploiting the most powerful neutrino beam ever built. Among its many capabilities it has the possibility to produce and then detect relativistic LDM particles that can pass through the shielding material, enter the detector and scatter off the atoms, whose relativistic recoils are detectable [42]. Due to highly intensified beam sources, large signal statistics is usually expected so that this sort of search strategy can allow for significant sensitivity to DM-induced signals, despite the feeble interaction of DM- SM particles. Assuming that DM is produced, DUNE will be capable to search for the relativistic scattering of LDM at the Near Detector, as it is close enough to the beam source to sample a substantial level of DM flux. The current strong involvement of the Latin American region in DUNE calls for successful participation on the DUNE DM search program.

\subsection{DM Phenomenology community in LA}

The Latin American DM community has a successful and long history of involvement in DM model building and DM phenomenology that started about three decades ago. The community is ripe to move to the next step of strongly supporting new developments of experimental groups within and beyond the currents efforts.

Latin American scientists have played a relevant role in both formulating and studying simplified WIMP-like DM scenarios [2-6 43-57], [58-78], [79-88]. At the same time, in many theoretical studies across high energy physics, astrophysics and cosmology Latin American communities have been dedicated to explore DM solutions based on axions, ALPs, dark photon particles [89.93], and more general ULDM [94-98], including a recent review [99].

Beyond the various non-thermal scenarios related to ULDM, other intriguing non-thermal and quasi-thermal DM scenarios for DM masses above the keV range have been extensively developed by the LA community. A challenging scenario, in the hope to find non-gravitational signals for dark matter, is expected for Feebly Interacting Massive Dark Matter Particles (FIMPs) in which the relic density is generated out of equilibrium by the so-called freeze-in mechanism. In this scenario, DM particles couple to the visible SM sector extremely weakly, so that they never entered chemical equilibrium. Instead, the DM particles were produced by decay or annihilation processes from the visible sector. The LA community has been particularly active in the exploration of FIMPs, pioneering studies on simplified models where the FIMP mechanism is realized [100-102] (see Ref. [103 104] for supersymmetric scenarios with gravitinos non-thermally produced). Additionally, the Latin American theory community has contributed to the understanding of the impact of non-sudden reheating on the UV freeze-in [105]. The only existing review on FIMP DM has a major contribution from Latin American scientsits [106.

Another non-thermal cosmological possibility is the Strongly Interacting Massive Particles (SIMP) mechanism. DM with strong self-interactions provides an appealing solution to several small-scale structure puzzles. Under the assumption that the coupling between DM and the SM particles is suppressed, SIMPs allow for a successful dark freeze-out through $N$-to- $N^{\prime}$ processes, where $N$ DM particles annihilate to $N^{\prime}$ of them, with $N>N^{\prime} \geq 2$. The Latin American community has been actively exploring this alternative production mechanism, whereas searching for strategies to probe it, in particular through astrophysical and cosmological observables [107]. A review on the different production regimes for SIMPs and general self-interacting DM has been produced by LA scientists [108].

Many generations of Latin American scientists has been trained in DM phenomenology and model building and the community is well prepared to continue contributing revolutionary ideas to the field, helping to build a stronger bridge between theory, cosmological observations and experiments.

\section{DM Community Activities}

Many activities, including schools and workshops have helped solidify interactions among the Latin American DM community and bring international experts to help train the younger generations. Notable examples include the ICTP-SAIFR regular workshops and meetings related to DM with high Latin American involvement and significant international participation. In addition, there is also the yearly Colombian workshop on DM (MOCa). Both initiatives arose in response to the community's desire to further strengthen efforts in DM related topics. In several Latin American countries like Argentina, Brazil, Chile, Colombia and Mexico there is a highly motivated critical mass of researchers working on different subjects of particle physics, cosmology, and astrophysics and future DM endeavours can capitalize on them. 


\subsection{Synergies}

The Dark Matter conundrum is at the boundary of particle physics, astro-particle physics and cosmology, and as such, interdisciplinary collaboration and cross-fertilization is needed.

The understanding of DM will demand signals from multiple experiments, together with compatibility with indirect measurements from DM annihilation and decay products. The general purpose Gamma Ray Observatories which aim to improve the existing catalogues of Gamma-ray sources in the sky have also the potential to discover DM and help understand its properties.

There is an important overlap both in technology and search opportunities between DM and neutrinos. From the experimental side the liquid argon detectors and related technologies are playing a crucial role both in DM searches, e.g. DarkSide, and the neutrino detectors, including the mega-project DUNE. Furthermore, the Skipper CDD technology is powerful in detecting low thresholds signals that are relevant for DM direct detection as well as for exploring neutrino properties.

The recent experimental success in observing gravitational waves has opened up a new avenue for observational cosmology, which will be further enhanced by the future LISA mission. Gravitational waves produced in phase transitions, possibly involving dark sectors, could be detected with the LISA observatory, regardless of the feeble strength of non-gravitational interactions between the dark and visible sector particles [105 109].

Baryon acoustic oscillations (BAO) are fluctuations in the density of the visible baryonic matter of the universe, caused by acoustic density waves in the primordial plasma of the early universe. BAO is one of the main evidences for DM and therefore its exploration has direct implications for dark sectors [110]. A possible experiment along this line - based on a Radio Telescope and leaded by Brazil - is BINGO (BAO from Integrated Neutral Gas Observations). It is designed to measure BAO at radio frequencies and its description and scientific scope can be found in the Cosmology chapter of this report.

The future Legacy Survey of Space and Time (LSST) will complement direct DM detection experiments by improving measurements of the local phase-space density of DM using precision astrometry of the Milky Way stars. For dark matter-baryon scattering, small-scale structure measurements with LSST can probe DM masses and cross sections outside the range accessible to direct detection experiments. For details see the Dark Matter white paper from LSST at https://lsstdarkmatter.github.io/

\subsection{Conclusions}

Astrophysical probes of DM require large underground facilities to have competitive limits in the exploration of various types of DM candidates. At present, the experimental activities in which the Latin American community is involved are based at European, US and Canadian underground laboratories. Researchers in Latin America are active in the DarkSide experiment, that has provided the best limits in the GeV region for DM-nucleon cross-sections, and has future plans to test DM candidates with annihilation cross sections close to the neutrino floor. The expertise and capabilities contributing to large direct DM search experiments could be further exploited by encouraging Latin American scientists to actively engage in the upcoming Global Argon DM Collaboration (GADMC), that is the long term future for liquid argon based WIMP-like DM searches. In the future there is the unique opportunity for an underground laboratory in the region: The prospective ANDES Agua Negra tunnel between Chile and Argentina. Such underground laboratory would be the only one of its kind in the southern hemisphere, with extraordinary capabilities for direct detection DM searches, as well as neutrino physics exploration, and will make the region a central international hub for underground experiments.

Other type of searches for DM candidates in the sub-GeV regime require very low energy threshold detectors. A new technology based on Skipper-CCD detectors can identify electron recoils at the level of one to few electrons from elastic scattering of MeV to GeV DM candidates. The SENSEI experiment at MINOS has currently set the stringent limits for light DM-electron scattering cross-sections in this mass range and it can also probe nuclear recoils successfully. In the near future, Latin American participation in DAMIC-M at Modane and SENSEI-100-gr-yr at SNOLAB will reach DM-electron scattering cross sections in the $10^{-40}$ to $10^{-41} \mathrm{~cm}^{2}$ sensitivity. It will be of interest to the LA community to capitalize on this experience and actively participate in the next generation of Skipper-CCDs detector based DM searches at the OSCURA experiment at SNOLAB, where two orders of magnitude gain in sensitivity for the DM-electron scattering cross sections are expected.

The Latin American community has acquired a strong expertise in Skipper-CCDs technology, and already plays a relevant role in a group of experiments based on it. Skipper-CCDs detectors are relevant not only for light 
DM searches, but also for exploring dark portals at detectors in the vicinity of nuclear reactor cores, such as the current, terrain-level experiment CONNIE in Brazil and the near future vIOLETA experiment Argentina. A new laboratory at UBA, Argentina is taking on the excellent opportunity to lead and train the next generation of Latin American experimenters in Skipper-CCDs technologies that are of use well beyond the DM efforts.

Latin America is playing a unique role in the next generation of gamma ray observatories, such as CTA South and SWGO, which offer excellent opportunities to perform DM experiments. This opens a remarkable possibility to the region of playing a leading role in indirect DM detection experiments.

The international particle physics community has been dedicated to search for DM candidates created in collisions at high energy collider experiments since the LEP era. At the same time, Latin American scientists have played an important role in Standard Model and beyond the standard model physics at colliders, including LEP, the Tevatron and now the LHC, and its upcoming High Luminosity (HL) upgrade. This opens an opportunity for the Latin American community to now engage in DM/dark sector collider searches. DM particles and long lived and/or feeble interacting particles, that may be related to extended DM sectors, may open the path to discoveries. An example of Latin American efforts is the CMS group in Colombia that has lead DM searches in various channels. The expertise and long term involvement in collider experiments in the region makes it possible for a broad group of young researchers to participate/lead DM searches and new analyses strategies at the HL-LHC run.

In addition to participating in large international projects such as the LHC, the Latin American community is also getting involved in the next neutrino mega-science project hosted by Fermilab: the Deep Underground Neutrino Experiment (DUNE). Although DUNE is an experiment primarily designed to explore neutrino properties, and its possible new interactions, it also offers a unique window into dark matter exploration.

Latin America has a large, mature theoretical community with vast expertise in DM model building and phenomenology, with active collaborations across the region. The theoretical community also leads the organization of schools and workshops that facilitate the training of the next generation. Such a strong theoretical effort will naturally evolve into an even deeper involvement in experimental activities ranging from direct and indirect detection experiments to specific accelerator based searches and new analysis techniques. The Latin American community is ripe to move to the next step in the exploration of DM and dark sector signals, and the theoretical community can bridge efforts, helping to prepare the next generation of DM/Dark sector experimentalists. 


\section{Bibliography}

[1] Richard Keith Ellis et al., "Physics Briefing Book: Input for the European Strategy for Particle Physics Update 2020," (10 2019), arXiv:1910.11775 [hep-ex]

[2] A. Masiero, S. Profumo, S.K. Vempati, and C.E. Yaguna, "Lepton flavor violation, neutralino dark matter and the reach of the LHC," JHEP 03, 046 (2004), arXiv:hep-ph/0401138

[3] Stefano Profumo and Carlos E. Yaguna, "A Statistical analysis of supersymmetric dark matter in the MSSM after WMAP," Phys. Rev. D 70, 095004 (2004), arXiv:hep-ph/0407036

[4] Nicolas Bernal, Abdelhak Djouadi, and Pietro Slavich, "The MSSM with heavy scalars," JHEP 07, 016 (2007), arXiv:0705.1496 [hep-ph]

[5] D. Aristizabal Sierra, Diego Restrepo, and Oscar Zapata, "Decaying Neutralino Dark Matter in Anomalous U(1)(H) Models," Phys. Rev. D 80, 055010 (2009), arXiv:0907.0682 [hep-ph]

[6] Nicolas Bernal and Andreas Goudelis, "Dark matter detection in the BMSSM," JCAP 03, 007 (2010), arXiv:0912.3905 [hep-ph]

[7] R. Ajaj et al. (DEAP), "Search for dark matter with a 231-day exposure of liquid argon using DEAP-3600 at SNOLAB," Phys. Rev. D 100, 022004 (2019), arXiv:1902.04048 [astro-ph.CO]

[8] E. Aprile et al. (XENON), "Constraining the spin-dependent WIMP-nucleon cross sections with XENON1T," Phys. Rev. Lett. 122, 141301 (2019), arXiv:1902.03234 [astro-ph.CO]

[9] E. Aprile et al. (XENON), "Dark Matter Search Results from a One Ton-Year Exposure of XENON1T," Phys. Rev. Lett. 121, 111302 (2018), arXiv:1805.12562 [astro-ph.CO]

[10] C. Amole et al. (PICO), "Dark Matter Search Results from the PICO-60 C ${ }_{3} F_{8}$ Bubble Chamber," Phys. Rev. Lett. 118, 251301 (2017), arXiv:1702.07666 [astro-ph.CO]

[11] H. Abdallah et al. (H.E.S.S.), "Search for dark matter annihilations towards the inner Galactic halo from 10 years of observations with H.E.S.S," Phys. Rev. Lett. 117, 111301 (2016), arXiv:1607.08142 [astro-ph.HE]

[12] M.L. Ahnen et al. (MAGIC, Fermi-LAT), "Limits to Dark Matter Annihilation Cross-Section from a Combined Analysis of MAGIC and Fermi-LAT Observations of Dwarf Satellite Galaxies," JCAP 02, 039 (2016), arXiv:1601.06590 [astro-ph.HE]

[13] D.S. Akerib et al. (LUX), "Improved Limits on Scattering of Weakly Interacting Massive Particles from Reanalysis of 2013 LUX Data," Phys. Rev. Lett. 116, 161301 (2016), arXiv:1512.03506 [astro-ph.CO]

[14] Giorgio Arcadi, Maíra Dutra, Pradipta Ghosh, Manfred Lindner, Yann Mambrini, Mathias Pierre, Stefano Profumo, and Farinaldo S. Queiroz, "The waning of the WIMP? A review of models, searches, and constraints," Eur. Phys. J. C 78, 203 (2018), arXiv:1703.07364 [hep-ph]

[15] David J. E. Marsh, "Axion Cosmology," Phys. Rept. 643, 1-79 (2016), arXiv:1510.07633 [astro-ph.CO]

[16] R.D. Peccei, "The Strong CP Problem," (1989) pp. 503-551

[17] Jihn E. Kim and Gianpaolo Carosi, "Axions and the Strong CP Problem," Rev. Mod. Phys. 82, 557-602 (2010), [Erratum: Rev.Mod.Phys. 91, 049902 (2019)], arXiv:0807.3125 [hep-ph]

[18] Steven Weinberg, "A New Light Boson?." Phys. Rev. Lett. 40, 223-226 (1978)

[19] Frank Wilczek, "Problem of Strong $P$ and $T$ Invariance in the Presence of Instantons," Phys. Rev. Lett. 40, 279-282 (1978)

[20] Marco Battaglieri et al., "US Cosmic Visions: New Ideas in Dark Matter 2017: Community Report," in U.S. Cosmic Visions: New Ideas in Dark Matter (2017) arXiv:1707.04591 [hep-ph]

[21] P. Agnes et al. (DarkSide), "Constraints on Sub-GeV Dark-Matter-Electron Scattering from the DarkSide50 Experiment," Phys. Rev. Lett. 121, 111303 (2018), arXiv:1802.06998 [astro-ph.CO] 
[22] P. Agnes et al. (DarkSide), "DarkSide-50 532-day Dark Matter Search with Low-Radioactivity Argon," Phys. Rev. D 98, 102006 (2018), arXiv:1802.07198 [astro-ph.CO]

[23] P. Agnes et al. (DarkSide), "Low-Mass Dark Matter Search with the DarkSide-50 Experiment," Phys. Rev. Lett. 121, 081307 (2018), arXiv:1802.06994 [astro-ph.HE]

[24] C.E. Aalseth et al., "DarkSide-20k: A 20 tonne two-phase LAr TPC for direct dark matter detection at LNGS," Eur. Phys. J. Plus 133, 131 (2018), arXiv:1707.08145 [physics.ins-det]

[25] J. Billard, L. Strigari, and E. Figueroa-Feliciano, "Implication of neutrino backgrounds on the reach of next generation dark matter direct detection experiments," Phys. Rev. D 89, 023524 (2014), arXiv:1307.5458 [hep-ph]

[26] P. Agnes et al. (DarkSide), "DarkSide-50 532-day Dark Matter Search with Low-Radioactivity Argon," Phys. Rev. D 98, 102006 (2018), arXiv:1802.07198 [astro-ph.CO]

[27] Liron Barak et al. (SENSEI), "SENSEI: Direct-Detection Results on sub-GeV Dark Matter from a New Skipper-CCD," (4 2020), arXiv:2004.11378 [astro-ph.CO]

[28] N. Castelló-Mor (DAMIC-M), "DAMIC-M Experiment: Thick, Silicon CCDs to search for Light Dark Matter," Nucl. Instrum. Meth. A 958, 162933 (2020), arXiv:2001.01476 [physics.ins-det]

[29] Aion Viana, Harm Schoorlemmer, Andrea Albert, Vitor de Souza, J. Patrick Harding, and Jim Hinton, "Searching for Dark Matter in the Galactic Halo with a Wide Field of View TeV Gamma-ray Observatory in the Southern Hemisphere," JCAP 12, 061 (2019), arXiv:1906.03353 [astro-ph.HE]

[30] Farinaldo S. Queiroz and Carlos E. Yaguna, "The CTA aims at the Inert Doublet Model," JCAP 02, 038 (2016), arXiv:1511.05967 [hep-ph]

[31] Andrés Flórez, Alfredo Gurrola, Will Johns, Jessica Maruri, Paul Sheldon, Kuver Sinha, and Savanna Rae Starko, "Anapole Dark Matter via Vector Boson Fusion Processes at the LHC," Phys. Rev. D 100, 016017 (2019), arXiv:1902.01488 [hep-ph]

[32] Siba Prasad Das, Jorge Fraga, and Carlos Avila, "Production of a light top-squark pair in association with a light non-SM Higgs boson within the NMSSM from proton-proton collisions at $\sqrt{s}=13 \mathrm{TeV}$ and 33 TeV," Int. J. Mod. Phys. A 34, 1950125 (2019), arXiv:1712.04395 [hep-ph]

[33] Carlos Avila, Andrés Flórez, Alfredo Gurrola, Dale Julson, and Savanna Starko, "Connecting particle physics and cosmology: Measuring the dark matter relic density in compressed supersymmetry models at the LHC," Phys. Dark Univ. 27, 100430 (2020), arXiv:1801.03966 [hep-ph]

[34] Alexandre Alves, Oscar J.P. Eboli, Giovanni Grilli Di Cortona, and Roberto R. Moreira, "Indirect and monojet constraints on scalar leptoquarks," Phys. Rev. D 99, 095005 (2019), arXiv:1812.08632 [hep-ph]

[35] Andrés Flórez, Luis Bravo, Alfredo Gurrola, Carlos Ávila, Manuel Segura, Paul Sheldon, and Will Johns, "Probing the stau-neutralino coannihilation region at the LHC with a soft tau lepton and a jet from initial state radiation," Phys. Rev. D 94, 073007 (2016), arXiv:1606.08878 [hep-ph]

[36] Vardan Khachatryan et al. (CMS), "Search for supersymmetry in the vector-boson fusion topology in proton-proton collisions at $\sqrt{s}=8 \mathrm{TeV}$," JHEP 11, 189 (2015), arXiv:1508.07628 [hep-ex]

[37] Vardan Khachatryan et al. (CMS), "Search for Dark Matter and Supersymmetry with a Compressed Mass Spectrum in the Vector Boson Fusion Topology in Proton-Proton Collisions at $\sqrt{s}=8 \mathrm{TeV}$," Phys. Rev. Lett. 118, 021802 (2017), arXiv:1605.09305 [hep-ex]

[38] Albert M Sirunyan et al. (CMS), "Search for supersymmetry with a compressed mass spectrum in the vector boson fusion topology with 1-lepton and 0-lepton final states in proton-proton collisions at $\sqrt{s}=$ 13 TeV," JHEP 08, 150 (2019), arXiv:1905.13059 [hep-ex]

[39] Albert M Sirunyan et al. (CMS), "Search for Supersymmetry with a Compressed Mass Spectrum in Events with a Soft $\tau$ Lepton, a Highly Energetic Jet, and Large Missing Transverse Momentum in Proton-Proton Collisions at $\sqrt{s}=$ TeV," Phys. Rev. Lett. 124, 041803 (2020), arXiv:1910.01185 [hep-ex]

[40] D. Akimov et al. (COHERENT), "Observation of Coherent Elastic Neutrino-Nucleus Scattering," Science 357, 1123-1126 (2017), arXiv:1708.01294 [nucl-ex]

[41] Alexis Aguilar-Arevalo et al. (CONNIE), "Search for light mediators in the low-energy data of the CONNIE reactor neutrino experiment," JHEP 04, 054 (2020), arXiv:1910.04951 [hep-ex]

[42] B. Abi et al. (DUNE), "Prospects for Beyond the Standard Model Physics Searches at the Deep Underground Neutrino Experiment," (8 2020), arXiv:2008.12769 [hep-ex] 
[43] Carlos E. Yaguna, "Gamma rays from the annihilation of singlet scalar dark matter," JCAP 03, 003 (2009), arXiv:0810.4267 [hep-ph]

[44] A. Goudelis, Y. Mambrini, and C. Yaguna, "Antimatter signals of singlet scalar dark matter," JCAP 12, 008 (2009), arXiv:0909.2799 [hep-ph]

[45] Laura Lopez Honorez and Carlos E. Yaguna, "The inert doublet model of dark matter revisited," JHEP 09, 046 (2010), arXiv:1003.3125 [hep-ph]

[46] Laura Lopez Honorez and Carlos E. Yaguna, "A new viable region of the inert doublet model," JCAP 01, 002 (2011), arXiv:1011.1411 [hep-ph]

[47] J.D. Ruiz-Alvarez, C.A. de S.Pires, Farinaldo S. Queiroz, D. Restrepo, and P.S. Rodrigues da Silva, "On the Connection of Gamma-Rays, Dark Matter and Higgs Searches at LHC," Phys. Rev. D 86, 075011 (2012), arXiv:1206.5779 [hep-ph]

[48] Sonja Esch, Michael Klasen, and Carlos E. Yaguna, "Detection prospects of singlet fermionic dark matter," Phys. Rev. D 88, 075017 (2013), arXiv:1308.0951 [hep-ph]

[49] R. Martínez, J. Nisperuza, F. Ochoa, and J.P. Rubio, "Scalar dark matter with CERN-LEP data and Z' search at the LHC in an $U(1)^{\prime}$ model," Phys. Rev. D 90, 095004 (2014), arXiv:1408.5153 [hep-ph]

[50] Chris Kelso, H.N. Long, R. Martinez, and Farinaldo S. Queiroz, "Connection of $g-2 \mu$, electroweak, dark matter, and collider constraints on 331 models," Phys. Rev. D 90, 113011 (2014), arXiv:1408.6203 [hep-ph]

[51] R. Martinez, J. Nisperuza, F. Ochoa, J.P. Rubio, and C.F. Sierra, "Scalar coupling limits and diphoton Higgs decay from LHC in an U(1)' model with scalar dark matter," Phys. Rev. D 92, 035016 (2015), arXiv:1411.1641 [hep-ph]

[52] R. Martinez and F. Ochoa, "Spin-independent interferences and spin-dependent interactions with scalar dark matter," JHEP 05, 113 (2016), arXiv:1512.04128 [hep-ph]

[53] Carolina Arbelaez, Robinson Longas, Diego Restrepo, and Oscar Zapata, "Fermion dark matter from SO(10) GUTs," Phys. Rev. D 93, 013012 (2016), arXiv:1509.06313 [hep-ph]

[54] Shunsaku Horiuchi, Oscar Macias, Diego Restrepo, Andres Rivera, Oscar Zapata, and Hamish Silverwood, "The Fermi-LAT gamma-ray excess at the Galactic Center in the singlet-doublet fermion dark matter model," JCAP 03, 048 (2016), arXiv:1602.04788 [hep-ph]

[55] Bhaskar Dutta, Guillermo Palacio, Jose D. Ruiz-Alvarez, and Diego Restrepo, "Vector Boson Fusion in the Inert Doublet Model," Phys. Rev. D 97, 055045 (2018), arXiv:1709.09796 [hep-ph]

[56] Carolina Arbeláez, Martin Hirsch, and Diego Restrepo, "Fermionic triplet dark matter in an SO(10)inspired left right model," Phys. Rev. D 95, 095034 (2017), arXiv:1703.08148 [hep-ph]

[57] Simonetta Filippi, William A. Ponce, and Luis A. Sanchez, "Dark matter from the scalar sector of 3-3-1 models without exotic electric charges," Europhys. Lett. 73, 142-148 (2006), arXiv:hep-ph/0509173

[58] Rodrigo von Marttens, Lucas Lombriser, Martin Kunz, Valerio Marra, Luciano Casarini, and Jailson Alcaniz, "Dark degeneracy I: Dynamical or interacting dark energy?." Phys. Dark Univ. 28, 100490 (2020), arXiv:1911.02618 [astro-ph.CO]

[59] Zahra Davari, Valerio Marra, and Mohammad Malekjani, "Cosmological constrains on minimally and non-minimally coupled scalar field models," Mon. Not. Roy. Astron. Soc. 491, 1920-1933 (2020), arXiv:1911.00209 [gr-qc]

[60] G. Pordeus-da Silva, R.C. Batista, and L.G. Medeiros, "Theoretical foundations of the reduced relativistic gas in the cosmological perturbed context," JCAP 06, 043 (2019), arXiv:1904.09904 [astro-ph.CO]

[61] Atri Bhattacharya, Arman Esmaili, Sergio Palomares-Ruiz, and Ina Sarcevic, "Update on decaying and annihilating heavy dark matter with the 6-year IceCube HESE data," JCAP 05, 051 (2019), arXiv:1903.12623 [hep-ph]

[62] R. von Marttens, L. Casarini, D.F. Mota, and W. Zimdahl, "Cosmological constraints on parametrized interacting dark energy," Phys. Dark Univ. 23, 100248 (2019), arXiv:1807.11380 [astro-ph.CO]

[63] Davi C. Rodrigues, Valerio Marra, Antonino del Popolo, and Zahra Davari, "Absence of a fundamental acceleration scale in galaxies," Nature Astron. 2, 668-672 (2018), arXiv:1806.06803 [astro-ph.GA]

[64] J.C. Montero, A. Romero, and B.L. Sánchez-Vega, "Axion dark matter in a 3-3-1 model," Phys. Rev. D 97, 063015 (2018), arXiv:1709.04535 [hep-ph] 
[65] W.S. Hipólito-Ricaldi, R.F. Marttens, J.C. Fabris, I.L. Shapiro, and L. Casarini, "On general features of warm dark matter with reduced relativistic gas," Eur. Phys. J. C 78, 365 (2018), arXiv:1706.08595 [astro-ph.CO]

[66] R.F. vom Marttens, L. Casarini, W. Zimdahl, W.S. Hipólito-Ricaldi, and D.F. Mota, "Does a generalized Chaplygin gas correctly describe the cosmological dark sector?." Phys. Dark Univ. 15, 114-124 (2017), arXiv:1702.00651 [astro-ph.CO]

[67] Davi C. Rodrigues, Antonino del Popolo, Valerio Marra, and Paulo L. C. de Oliveira, "Evidence against cuspy dark matter haloes in large galaxies," Mon. Not. Roy. Astron. Soc. 470, 2410-2426 (2017), arXiv:1701.02698 [astro-ph.GA]

[68] Álefe O.F. de Almeida, Oliver F. Piattella, and Davi C. Rodrigues, "A method for evaluating models that use galaxy rotation curves to derive the density profiles," Mon. Not. Roy. Astron. Soc. 462, 2706-2714 (2016), arXiv:1605.04269 [astro-ph.GA]

[69] L. Casarini, S.A. Bonometto, E. Tessarotto, and P. S. Corasaniti, "Extending the Coyote emulator to dark energy models with standard $w_{0}-w_{a}$ parametrization of the equation of state," JCAP 08, 008 (2016), arXiv:1601.07230 [astro-ph.CO]

[70] C. Pigozzo, S. Carneiro, J.S. Alcaniz, H.A. Borges, and J.C. Fabris, "Evidence for cosmological particle creation?." JCAP 05, 022 (2016), arXiv:1510.01794 [astro-ph.CO]

[71] O.F. Piattella, L. Casarini, J.C. Fabris, and J. A. de Freitas Pacheco, "Dark matter velocity dispersion effects on CMB and matter power spectra," JCAP 02, 024 (2016), arXiv:1507.00982 [astro-ph.CO]

[72] Hermano Velten, Thiago R. P. Caramês, Júlio C. Fabris, Luciano Casarini, and Ronaldo C. Batista, "Structure formation in a $\Lambda$ viscous CDM universe," Phys. Rev. D 90, 123526 (2014), arXiv:1410.3066 [astro-ph.CO]

[73] Davi C. Rodrigues, Paulo L. de Oliveira, Júlio C. Fabris, and Gianfranco Gentile, "Modified gravity models and the central cusp of dark matter haloes in galaxies," Mon. Not. Roy. Astron. Soc. 445, 3823-3838 (2014), arXiv:1409.7524 [astro-ph.GA]

[74] Nicolás Bernal, Jaime E. Forero-Romero, Raghuveer Garani, and Sergio Palomares-Ruiz, "Systematic uncertainties from halo asphericity in dark matter searches," JCAP 09, 004 (2014), arXiv:1405.6240 [astro-ph.CO]

[75] Arman Esmaili and Pasquale Dario Serpico, "Are IceCube neutrinos unveiling PeV-scale decaying dark matter?." JCAP 11, 054 (2013), arXiv:1308.1105 [hep-ph]

[76] Arman Esmaili and Yasaman Farzan, "A Novel Method to Extract Dark Matter Parameters from Neutrino Telescope Data," JCAP 04, 007 (2011), arXiv:1011.0500 [hep-ph]

[77] J.K. Mizukoshi, C.A. de S.Pires, F.S. Queiroz, and P.S. Rodrigues da Silva, "WIMPs in a 3-3-1 model with heavy Sterile neutrinos," Phys. Rev. D 83, 065024 (2011), arXiv:1010.4097 [hep-ph]

[78] Arman Esmaili and Yasaman Farzan, "On the Oscillation of Neutrinos Produced by the Annihilation of Dark Matter inside the Sun," Phys. Rev. D 81, 113010 (2010), arXiv:0912.4033 [hep-ph]

[79] A.V. Penacchioni, O. Civitarese, and C.R. Argüelles, "Testing dark matter distributions by neutrino-dark matter interactions," Eur. Phys. J. C 80, 183 (2020)

[80] Fernando Izaurieta, Samuel Lepe, and Omar Valdivia, "The Spin Tensor of Dark Matter and the Hubble Parameter Tension," Phys. Dark Univ. 30, 100662 (2020), arXiv:2004.13163 [gr-qc]

[81] Germán A. Gómez-Vargas, Daniel E. López-Fogliani, Carlos Muñoz, and Andres D. Perez, "MeV-GeV $\gamma$ ray telescopes probing axino LSP/gravitino NLSP as dark matter in the $\mu v$ SSM," JCAP 01, 058 (2020), arXiv:1911.03191 [hep-ph]

[82] D. Aristizabal Sierra, Bhaskar Dutta, Shu Liao, and Louis E. Strigari, "Coherent elastic neutrino-nucleus scattering in multi-ton scale dark matter experiments: Classification of vector and scalar interactions new physics signals," JHEP 12, 124 (2019), arXiv:1910.12437 [hep-ph]

[83] Paola Arias, Nicolás Bernal, Alan Herrera, and Carlos Maldonado, "Reconstructing Non-standard Cosmologies with Dark Matter," JCAP 10, 047 (2019), arXiv:1906.04183 [hep-ph]

[84] Julio Leite, América Morales, José W.F. Valle, and Carlos A. Vaquera-Araujo, "Scotogenic dark matter and Dirac neutrinos from unbroken gauged B-L symmetry," Phys. Lett. B 807, 135537 (2020), arXiv:2003.02950 [hep-ph] 
[85] H. Hernández-Arellano, M. Napsuciale, and S. Rodríguez, "Spin-one dark matter and gamma ray signals from the galactic center," JHEP 08, 106 (2020), arXiv:1911.01604 [hep-ph]

[86] Cesar Bonilla, Leon M.G. de la Vega, J.M. Lamprea, Roberto A. Lineros, and Eduardo Peinado, "Fermion Dark Matter and Radiative Neutrino Masses from Spontaneous Lepton Number Breaking," New J. Phys. 22, 033009 (2020), arXiv:1908.04276 [hep-ph]

[87] T. Faber, Y. Liu, W. Porod, and J. Jones-Pérez, "Revisiting neutrino and sneutrino dark matter in natural SUSY scenarios," Phys. Rev. D 101, 055029 (2020), arXiv:1909.11686 [hep-ph]

[88] M.E. Cabrera, J.A. Casas, A. Delgado, and S. Robles, "Generalized Blind Spots for Dark Matter Direct Detection in the 2HDM," JHEP 02, 166 (2020), arXiv:1912.01758 [hep-ph]

[89] Andreas Ringwald, "Alternative dark matter candidates: Axions," PoS NOW2016, 081 (2016), arXiv:1612.08933 [hep-ph]

[90] Sz. Borsanyi et al., "Calculation of the axion mass based on high-temperature lattice quantum chromodynamics," Nature 539, 69-71 (2016), arXiv:1606.07494 [hep-lat]

[91] Alexandre Alves, Daniel A. Camargo, Alex G. Dias, Robinson Longas, Celso C. Nishi, and Farinaldo S. Queiroz, "Collider and Dark Matter Searches in the Inert Doublet Model from Peccei-Quinn Symmetry," JHEP 10, 015 (2016), arXiv:1606.07086 [hep-ph]

[92] Peter W. Graham, Igor G. Irastorza, Steven K. Lamoreaux, Axel Lindner, and Karl A. van Bibber, "Experimental Searches for the Axion and Axion-Like Particles," Ann. Rev. Nucl. Part. Sci. 65, 485-514 (2015), arXiv:1602.00039 [hep-ex]

[93] A.G. Dias, A.C.B. Machado, C.C. Nishi, A. Ringwald, and P. Vaudrevange, "The Quest for an Intermediate-Scale Accidental Axion and Further ALPs," JHEP 06, 037 (2014), arXiv:1403.5760 [hep-ph]

[94] Diego Blas, Diana Lopez Nacir, and Sergey Sibiryakov, "Ultralight Dark Matter Resonates with Binary Pulsars," Phys. Rev. Lett. 118, 261102 (2017), arXiv:1612.06789 [hep-ph]

[95] Diana López Nacir and Federico R. Urban, "Vector Fuzzy Dark Matter, Fifth Forces, and Binary Pulsars," JCAP 10, 044 (2018), arXiv:1807.10491 [astro-ph.CO]

[96] Diego Blas, Diana López Nacir, and Sergey Sibiryakov, "Secular effects of ultralight dark matter on binary pulsars," Phys. Rev. D 101, 063016 (2020), arXiv:1910.08544 [gr-qc]

[97] Juan Manuel Armaleo, Diana López Nacir, and Federico R. Urban, "Binary pulsars as probes for spin-2 ultralight dark matter," JCAP 01, 053 (2020), arXiv:1909.13814 [astro-ph.HE]

[98] Elías Castellanos, Celia Escamilla-Rivera, and Jorge Mastache, "Is a Bose-Einstein Condensate a good candidate for Dark Matter? A test with Galaxy Rotation Curves," Int. J. Mod. Phys. D 29, 2050063 (2020), arXiv:1910.03791 [gr-qc]

[99] Elisa G.M. Ferreira, "Ultra-Light Dark Matter," (5 2020), arXiv:2005.03254 [astro-ph.CO]

[100] Carlos E. Yaguna, "The Singlet Scalar as FIMP Dark Matter," JHEP 08, 060 (2011), arXiv:1105.1654 [hep-ph]

[101] Emiliano Molinaro, Carlos E. Yaguna, and Oscar Zapata, "FIMP realization of the scotogenic model," JCAP 07, 015 (2014), arXiv:1405.1259 [hep-ph]

[102] Nicolás Bernal, Catarina Cosme, Tommi Tenkanen, and Ville Vaskonen, "Scalar singlet dark matter in non-standard cosmologies," Eur. Phys. J. C 79, 30 (2019), arXiv:1806.11122 [hep-ph]

[103] Ki-Young Choi, Diego Restrepo, Carlos E. Yaguna, and Oscar Zapata, "Indirect detection of gravitino dark matter including its three-body decays," JCAP 10, 033 (2010), arXiv:1007.1728 [hep-ph]

[104] Diego Restrepo, Marco Taoso, J.W.F. Valle, and Oscar Zapata, "Gravitino dark matter and neutrino masses with bilinear R-parity violation," Phys. Rev. D 85, 023523 (2012), arXiv:1109.0512 [hep-ph]

[105] Nicolás Bernal, Javier Rubio, and Hardi Veermäe, "UV Freeze-in in Starobinsky Inflation," (6 2020), arXiv:2006.02442 [hep-ph]

[106] Nicolás Bernal, Matti Heikinheimo, Tommi Tenkanen, Kimmo Tuominen, and Ville Vaskonen, "The Dawn of FIMP Dark Matter: A Review of Models and Constraints," Int. J. Mod. Phys. A 32, 1730023 (2017), arXiv:1706.07442 [hep-ph]

[107] Nicolás Bernal, "Boosting Freeze-in through Thermalization," (5 2020), arXiv:2005.08988 [hep-ph]

[108] Nicolas Bernal, Xiaoyong Chu, Camilo Garcia-Cely, Thomas Hambye, and Bryan Zaldivar, "Production Regimes for Self-Interacting Dark Matter," JCAP 03, 018 (2016), arXiv:1510.08063 [hep-ph] 
[109] Juan P. Beltrán Almeida, Nicolás Bernal, Javier Rubio, and Tommi Tenkanen, "Hidden Inflaton Dark Matter," JCAP 03, 012 (2019), arXiv:1811.09640 [hep-ph]

[110] B. Wang, E. Abdalla, F. Atrio-Barandela, and D. Pavon, "Dark Matter and Dark Energy Interactions: Theoretical Challenges, Cosmological Implications and Observational Signatures," Rept. Prog. Phys. 79, 096901 (2016), arXiv:1603.08299 [astro-ph.CO] 


\section{Neutrinos}

Alfredo Aranda (U. de Colima, Mexico)

Jorge Molina (U. Nacional de Asunción, Paraguay)

Harold Yepes Ramírez (YTU, Ecuador)

\subsection{Introduction}

Neutrinos are key to solving open questions in our understanding of matter and the subatomic world. Latin America's participation in international neutrino experiments is relatively new. Nevertheless, it is at the core of the efforts in Latin America to participate in future local and non-local research infrastructures for global neutrino data analysis networks, through upgrades and scaling of already existing infrastructures or the development of new ones.

The neutrino puzzle is still not fully solved. Current main concerns regarding neutrinos may fall into two categories: neutrino properties and the role of neutrino as probes of astrophysical processes. In this respect, active topics address fundamental questions, such as precision measurements of neutrino oscillation parameters (mixing angles), the leptonic CP-violation phase $\left(\delta_{C P}\right)$ of the mixing matrix, the ordering of the neutrino mass states (Neutrino Mass Hierarchy - NMH), their Dirac or Majorana nature, the absolute neutrino mass scale, identification of possible astrophysical sources, and new neutrino states (such as a sterile neutrino). In particular, the main unknown parameters being pursued are: to resolve the $\theta_{23}$ octant degenracy, the NMH and $\delta_{C P}$. Notwithstanding the concerns still to be solved about neutrinos, there are potential applications of neutrino related-technology with some prototypes already running.

\subsubsection{Neutrino oscillations, mass hierarchy and leptonic phase}

Neutrino oscillations are described by the Pontecorvo-Maki-Nakagawa-Sakata (PMNS) mixing matrix, which relates the flavour eigenstates $\left(v_{e}, v_{\mu}, v_{\tau}\right)$ to mass eigenstates $\left(v_{1}, v_{2}, v_{3}\right)$ [1], as shown in Equation (5.1) for the Dirac neutrino case:

$$
\left[\begin{array}{c}
v_{e} \\
v_{\mu} \\
v_{\tau}
\end{array}\right]=\left[\begin{array}{ccc}
U_{e 1} & U_{e 2} & U_{e 3} \\
U_{\mu 1} & U_{\mu 2} & U_{\mu 3} \\
U_{\tau 1} & U_{\tau 2} & U_{\tau 3}
\end{array}\right]\left[\begin{array}{c}
v_{1} \\
v_{2} \\
v_{3}
\end{array}\right]
$$

The simplest approximation to the oscillation probability between two neutrino states $(2 v)$, having a neutrino state $v_{\alpha}$ being detected as $v_{\beta}$ is:

$$
P_{2 v}\left(v_{\alpha} \rightarrow v_{\beta}\right)=\sin ^{2}(2 \theta) \sin ^{2}\left(\frac{\Delta m_{i j}^{2} L}{4 E_{v}}\right),
$$

with $\Delta m_{i j}^{2}=m_{i}^{2}-m_{j}^{2}$ ( $m_{j}$ is the mass of the $j$-th neutrino mass eigenstate). Equation (5.2) is able to explain almost all the data available on neutrino oscillation parameters (except Short Baseline (SBL) anomalies) even in a 3-flavour neutrino $(3 v)$ scenario [2]. The PMNS matrix can be parametrized as the product of three independent rotations, through three mixing angles $\left(\theta_{23}, \theta_{13}, \theta_{12}\right)$, where the second (unitary) rotation depends on a phase $\left(\delta_{C P}\right)$, and of a diagonal matrix of phases $(P)$ : 


$$
\begin{aligned}
U_{P M N S} & =\left[\begin{array}{ccc}
1 & 0 & 0 \\
0 & \cos \theta_{23} & \sin \theta_{23} \\
0 & -\sin \theta_{23} & \cos \theta_{23}
\end{array}\right]\left[\begin{array}{ccc}
\cos \theta_{13} & 0 & e^{i \delta_{C P} \sin \theta_{13}} \\
0 & 1 & 0 \\
-e^{i \delta_{C P}} \sin \theta_{13} & 0 & \cos \theta_{13}
\end{array}\right]\left[\begin{array}{ccc}
\cos \theta_{12} & \sin \theta_{12} & 0 \\
-\sin \theta_{12} & \cos \theta_{12} & 0 \\
0 & 0 & 1
\end{array}\right] P \\
& =\left[\begin{array}{ccc}
c_{12} c_{13} & s_{12} c_{13} & e^{i \delta} s_{13} \\
-s_{12} c_{23}-e^{i \delta} s_{13} c_{12} s_{23} & c_{12} c_{23}-e^{i \delta} s_{13} s_{12} s_{23} & s_{23} c_{13} \\
s_{12} s_{23}-e^{i \delta} s_{13} c_{12} c_{23} & -c_{12} s_{23}-e^{i \delta} s_{13} s_{12} c_{23} & c_{23} c_{13}
\end{array}\right] P
\end{aligned}
$$

where the first, second and third matrices represent the accessible regions to atmospheric, reactor and solar neutrino fluxes, respectively, and $P$ is a unitary matrix for the Dirac neutrino case or a diagonal matrix (depending on two phases) for the Majorana neutrino case [3]. The three mixing angles have all been measured and current efforts are focused on precision measurements. In contrast $\delta_{C P}$ is still unknown. In addition to these parameters, there are two independent mass-squared differences $\Delta m^{2}$ scales present in the puzzle $\left(\Delta m_{21}^{2}\right.$, $\Delta m_{32}^{2}$ ). The remaining mass difference is given by:

$$
\Delta m_{31}^{2}=\Delta m_{32}^{2}+\Delta m_{21}^{2}
$$

One usually defines two possibilities for the ordering of the neutrino masses: a "Normal Hierarchy" (NH) with $m_{3}>m_{2}>m_{1}$ and an "Inverted Hierarchy" with $m_{2}>m_{1}>m_{3}$. In the latter case, $\Delta m_{31}^{2}$ and $\Delta m_{32}^{2}$ are both negative, and can be precisely measured in reactor experiments [4], and represent the mass difference between the heaviest and lightest neutrino or the opposite. The $3 v$ oscillation parameters from fits based on most recent data available are summarized in Table 5.1. [5] [6].

Table 5.1: The $3 v$ scenario fit to global data (July 2020) obtained with the inclusion of the tabulated $\chi^{2}$ data on atmospheric neutrinos provided by the Super-K Collaboration. The best fit point (bfp) is shown as the central value.

\begin{tabular}{ccccc}
\hline Parameter & bfp $\pm 1 \sigma(\mathrm{NH})$ & $3 \sigma$ range $(\mathrm{NH})$ & bfp $\pm 1 \sigma(\mathrm{IH})$ & $3 \sigma$ range $(\mathrm{IH})$ \\
\hline $\sin ^{2} \theta_{12}$ & $0.304_{-0.012}^{+0.012}$ & $0.269 \rightarrow 0.343$ & $0.304_{-0.012}^{+0.013}$ & $0.269 \rightarrow 0.343$ \\
$\theta_{12} /^{\circ}$ & $33.44_{-0.74}^{+0.77}$ & $31.27 \rightarrow 35.86$ & $33.45_{-0.75}^{+0.78}$ & $31.27 \rightarrow 35.87$ \\
$\sin ^{2} \theta_{23}$ & $0.573_{-0.020}^{+0.016}$ & $0.415 \rightarrow 0.616$ & $0.575_{-0.019}^{+0.016}$ & $0.419 \rightarrow 0.617$ \\
$\theta_{23} /^{\circ}$ & $49.2_{-1.2}^{+0.9}$ & $40.1 \rightarrow 51.7$ & $49.3_{-1.1}^{+0.9}$ & $40.3 \rightarrow 51.8$ \\
$\sin ^{2} \theta_{13}$ & $0.02219_{-0.00062}^{+0.00063}$ & $0.02032 \rightarrow 0.02410$ & $0.02238_{-0.00062}^{+0.00063}$ & $0.02052 \rightarrow 0.02428$ \\
$\theta_{13} /^{\circ}$ & $8.57_{-0.12}^{+0.12}$ & $8.20 \rightarrow 8.93$ & $8.60_{-0.12}^{+0.12}$ & $8.24 \rightarrow 8.96$ \\
$\delta_{C P} /^{\circ}$ & $197_{-24}^{+27}$ & $120 \rightarrow 369$ & $282_{-30}^{+26}$ & $193 \rightarrow 352$ \\
$\frac{\Delta m_{21}^{2}}{10^{-5} e V^{2}}$ & $7.42_{-0.20}^{+0.21}$ & $6.82 \rightarrow 8.04$ & $7.42_{-0.20}^{+0.21}$ & $6.82 \rightarrow 8.04$ \\
$\frac{\Delta m_{3 l}^{2}}{10^{-3} e V^{2}}$ & $+2.517_{-0.028}^{+0.026}$ & $+2.435 \rightarrow+2.598$ & $-2.498_{-0.028}^{+0.028}$ & $-2.581 \rightarrow-2.414$ \\
\hline
\end{tabular}

As shown, the best fit is obtained for $\mathrm{NH}$ data $\left(\Delta m_{31}^{2}>0\right)$, while IH data is disfavoured with a difference of $\Delta \chi^{2}=7.1$ between both hypothesis, relative to the corresponding local minimum. Additionally, it is possible to observe $\Delta m_{32}^{2}, \Delta m_{31}^{2}>0$ for $\mathrm{NH}$ and $\Delta m_{31}^{2}, \Delta m_{32}^{2}<0$ for $\mathrm{IH}$.

Determination of $\delta_{C P}$ is being searched through enormous efforts of future upgrades to artificial neutrino sources in the main accelerator facilities worldwide at Fermilab, JPARC and Protvino. Outstanding synergies are taking place with leading expectations from the analysis of data collected by experiments such as MINOS, NOVA and T2K [5]. Nonetheless, very competitive sensitivity is also estimated by the next generation of experiments such as DUNE [7] and KM3NeT-ORCA (the P2O side project [8]), with planned upgrades on the beam at different power and exposures, in turn being suited for precision on oscillation parameters with $\mathrm{NH}$. P2O, with a sufficiently long beam exposure $(\sim 4$ year at $450 \mathrm{~kW})$, can reach a $2 \sigma$ sensitivity to $\delta_{C P}$, comparable with the projected sensitivity of NOvA and T2K. Recently, T2K presented results that exclude the complete absence of $\mathrm{CP}$ violation at $95 \% \mathrm{CL}[\overline{9}]$. An advanced phase of $\mathrm{P} 2 \mathrm{O}$ would give a $6 \sigma$ sensitivity to $\delta_{C P}$ after 10 years 
of operation at $450 \mathrm{~kW}$, competitive with the projected sensitivity of also next generation experiments DUNE and $\mathrm{T} 2 \mathrm{~K} / \mathrm{T} 2 \mathrm{HK}$.

Table 5.2 presents a list of earth-based neutrino factories and detectors with current and possible future Latin American participation, where (-) stands for no detector planned yet.

Table 5.2: Neutrino sources and detectors with Latin American participation. The tags stands for: ${ }^{\S}$ unused (but of interest for local small/large scale experiment), ${ }^{\dagger}$ already in use (by local/international experiment), ${ }^{\ddagger}$ planned (for international experiment). For reactors ( $\bar{v}$ flux) [10] thermal power is assumed. Baselines stand for running (or approved) experiments where computed/expected fluxes are also indicated. Space-based neutrino sources availability are assumed by default. POT stands for Protons On Target.

\begin{tabular}{|c|c|c|c|c|c|c|}
\hline Source & Detector & $\mathrm{L}[\mathrm{m}]$ & $P[G W]$ & $\phi_{v}\left[\mathrm{~s}^{-1}\right]$ & Operator & Type \\
\hline${ }^{\S}$ Embalse $(\mathrm{AR})$ & - & - & 2.1 & - & NASA & PHWR \\
\hline${ }^{\S}$ Atucha I (AR) & - & - & 1.2 & - & NASA & PHWR \\
\hline 'Atucha II (AR) & $v$ IOLETA & $8(12)$ & 2.2 & $9.0(4.0) \times 10^{20}$ & NASA & PHWR \\
\hline${ }^{\S}$ Angra I (BR) & - & - & 1.9 & - & Eletronuclear & PWR \\
\hline Angra II (BR) & $v$-Angra/CONNIE & 30 & 3.8 & $1.21 \times 10^{20}$ & Eletronuclear & PWR \\
\hline${ }^{\S}$ Laguna Verde I (MX) & - & - & 2.3 & - & CFE & BWR \\
\hline${ }^{\S}$ Laguna Verde II (MX) & - & - & 2.3 & - & CFE & BWR \\
\hline §Yangjiang/Taishan (CN) & JUNO & $5.3 \times 10^{4}$ & 26.61 & $\sim 10^{20}$ & YJNPC/TNPJVC & PWR \\
\hline${ }^{\dagger} \mathrm{NuMI}$ (US) & MINERvA/NOvA & $1.0 \times 10^{3}$ & $0.7 \times 10^{-3}$ & $\sim 10^{20}(\mathrm{POT})$ & Fermilab & LINAC \\
\hline †BNF (US) & DUNE & $1.3 \times 10^{6}$ & $2.4 \times 10^{-3}$ & $\sim 10^{21}(\mathrm{POT})$ & Fermilab & LINAC \\
\hline$\ddagger$ J-PARC (JP) & Hyper-K & $2.95 \times 10^{5}$ & $1.3 \times 10^{-3}$ & $\sim 10^{22}(\mathrm{POT})$ & J-PARC & LINAC \\
\hline Protvino (RU) & KM3NeT-ORCA & $2.6 \times 10^{6}$ & $4.5 \times 10^{-4}$ & $\sim 10^{20}(\mathrm{POT})$ & Protvino & LINAC \\
\hline
\end{tabular}

The above equations refer to the standard 3-flavour neutrino scenario. However, for a (3+1)-flavour scenario (e.g., addition of a hypothetical sterile neutrino), Equation (5.1) can be modified accordingly by adding new flavour $\left(v_{s}\right)$ and mass $\left(v_{4}\right)$ eigenstates connected through the $\left[U_{s 1}, U_{s 2}, U_{s 3}\right]$ row vector in the PMNS matrix, in order to give room to include a fourth flavour, hence, obtaining a $4 \times 4$ PMNS matrix and three independent squared-mass differences $\Delta m^{2}$ where $U_{e 4}^{2}+U_{\mu 4}^{2}+U_{\tau 4}^{2}+U_{s 4}^{2}=1$, is a PMNS unitarity condition.

Additional details about the main challenges in neutrino oscillations, related to the determination of the mass hierarchy and leptonic CP phase for experiments with Latin American participation, are reviewed in Section 5.2

\subsubsection{Neutrino masses and nature}

Neutrinoless Double Beta Decay $(\beta \beta 0 v)$ searches have the potential to solve the questions related to the neutrino nature and absolute mass scale. This process requires the neutrino to be its own antiparticle, thus neutrinos are Majorana particles, $L$ ( $R$ subindexes denoting the Left- or Right-handed nature of neutrinos). Experimental searches are looking for the so-called effective (Majorana) mass [11]:

$$
\left\langle m_{\beta \beta}\right\rangle=\left|\sum_{i=1}^{3} U_{e i}^{2} m_{i}\right| .
$$

In the case that $\beta \beta 0 \mathrm{v}$ is mediated by light neutrino exchange, Equation (5.5) can be linked to the corresponding isotope half-life $\left(T_{1 / 2}^{0 v}\right)$ as a function of either $\left\langle m_{\beta \beta}\right\rangle$ and the nuclear matrix element $\left(M^{0 v}\right)$ (describing all the nuclear structure effects):

$$
T_{1 / 2}^{0 v}=G^{0 v}(Q, Z)\left|M^{0 v}\right|^{2}\left(\frac{\left\langle m_{\beta \beta}\right\rangle}{m_{e}}\right)^{2},
$$

where $G^{0 v}(Q, Z)$ corresponds to the lepton phase-space (kinematic) factor (function of the $\mathrm{Q}$-value of the decay, charge and mass of the final state nucleus). Figure 5.1 extracted from [12], represents the allowed regions plots in terms of the allowed range $m_{v_{e}}$ (from ${ }^{3} \mathrm{He} \beta$-decay) and $m_{e e}\left(m_{\beta \beta}\right)$ (from $\beta \beta 0 v$ decay) as a function of $m_{\text {light }} \equiv m_{0}$.

The ranges are obtained by projecting the results of the global analysis of oscillation data, with the exclusion of the tabulated $\chi^{2}$ data on atmospheric neutrinos provided by Super-K. The region for each ordering is defined 

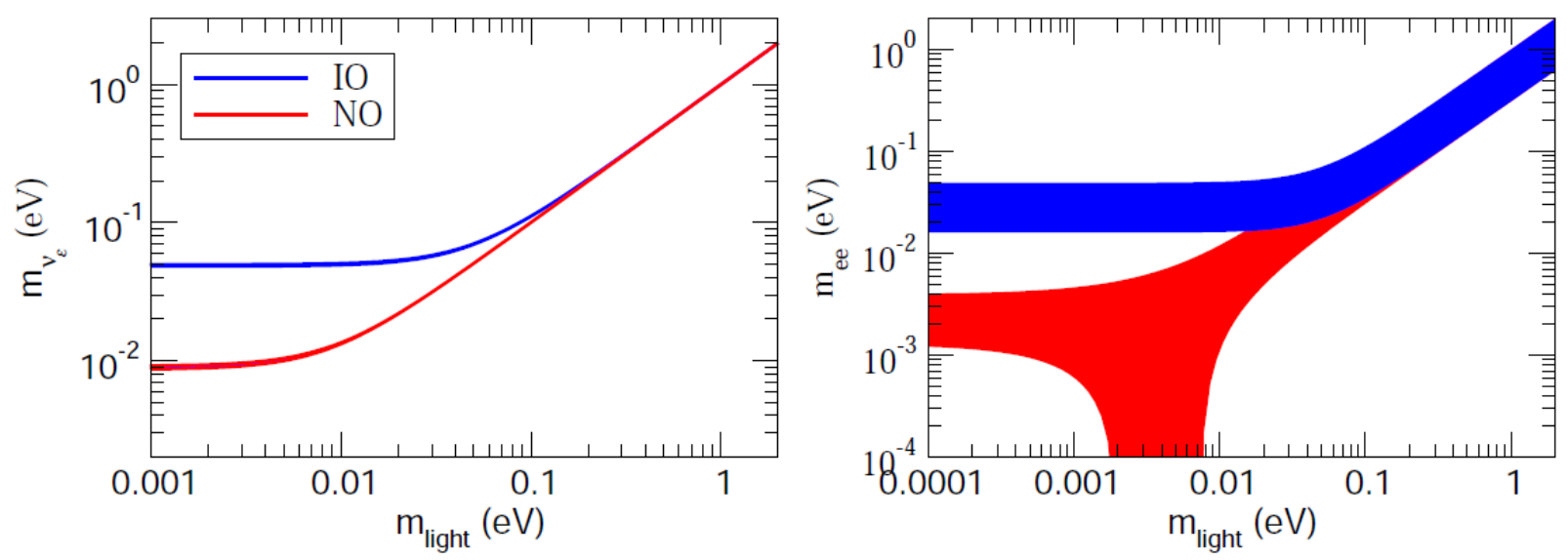

Figure 5.1: Allowed regions at 95\% of Confidence Level ranges, one degree of freedom, in the $3 v$ mixing scenario as a function of the lightest neutrino. Left: neutrino mass observable in ${ }^{3} \mathrm{He} \beta$-decay. Right: neutrino mass expected in $\beta \beta 0 \mathrm{v}$.

with respect to its local minimum. To date, the best neutrino mass limit is set by the KamLand Zen experiment, with $m_{\beta \beta}=50-160 \mathrm{meV}$, the best half-life sensitivity is set by the GERDA experiment, with $T_{1 / 2}>11 \times 10^{25}$ years $(90 \%$ Confidence Level). Scalability of this kind of experiments projects sensitivities below $10 \mathrm{meV}$, indeed covering the allowed parameter space for $\mathrm{IH}$ as in Figure 5.1

\subsubsection{Astrophysical probes}

A lot of progress has been made in the last few years in the design of experiments, techniques and sensitivities aimed at unveiling neutrino properties, their nature, and usefulness as a probe, with huge remarkable achievements. This is due to the strategic cooperation between experimentalists, theorists and funding capabilities. The discovery of the TXS 0506+056 Blazar as the first extragalactic neutrino source by the IceCube Collaboration [13] boosted the potential of Neutrino Observatories to complete the picture of astrophysical neutrinos. The complementary observation of whole the sky, by the Mediterranean and South Pole Neutrino Observatories, KM3NeT and IceCube, is strategic and crucial in the current understanding of the High-Energy (HE) neutrino astrophysical phenomenon. Figure 5.2 extracted from [14], represents the expected sensitivity to point-like neutrino sources by assuming a generic, unbroken power law energy spectrum proportional to $\mathrm{E}^{-2}$ flux (compatible with IceCube findings) as a function of the declination angle at $5 \sigma$ significance, for both of the aforementioned observatories.

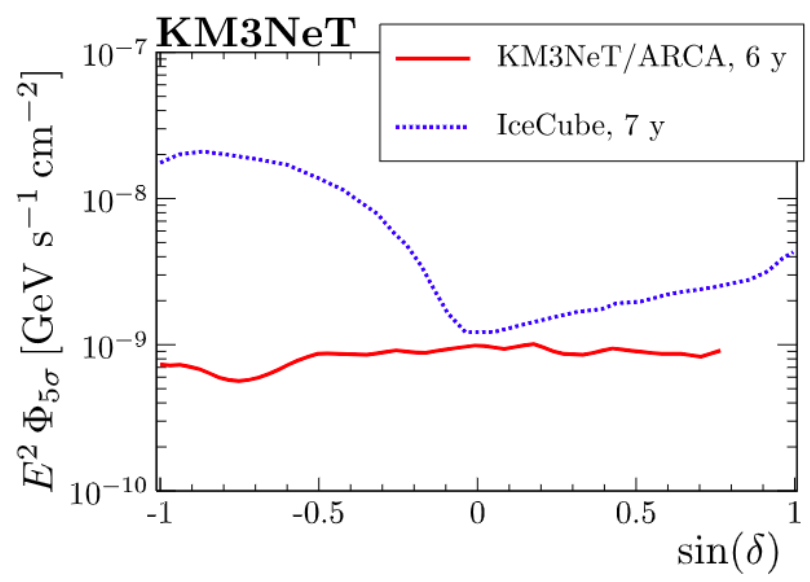

Figure 5.2: Discovery flux $\left(\Phi_{5 \sigma}\right)$ as a function of the source declination angle $(\delta)$ for the Mediterranean (twosided gaussian probability) and South Pole (one-sided gaussian probability) very-large volume HE Neutrino Observatories. Convention in gaussian probabilities for KM3NeT impacts weakly on the results $(<4 \%)$. 
As can be seen, an excellent sensitivity in the Southern Hemisphere can be reached with KM3NeT for an observation time of six years, comparable to results reported by IceCube in similar conditions with seven years of data of all sky search. The detection media (sea water and polar ice) play a fundamental role in the scientific goals of such experiments. The better the understanding of light propagation in the medium the better detectors performance is achieved (e.g., angular resolution, effective area). In ice there is less light absorption so there is better energy reconstruction. In water there is less scattering thus better pointing accuracy (point source identification). The location of $\mathrm{KM} 3 \mathrm{NeT}$ in the Northern hemisphere provides a full sky coverage, visibility of Galactic Plane, Galactic Center, and a $1.5 \mathrm{sr}$ common view per day. The contribution to strategic activities like this plays an important role and sets challenges regarding the know-how. Contribution from Latin America to $\mathrm{KM} 3 \mathrm{NeT}$, where an Optical Calibration System (OCS) is of special interest, a device that might be extended to other Water Cherenkov Detectors (WCD). The arrival of the KM3NeT Collaboration to Latin America guarantees at least the participation in one of these strategic infrastructures optimized for HE astrophysical neutrino studies, joining to the workforce on this strategic area in the world. Additionally, further results of KM3NeT regarding sensitivity to diffuse neutrino flux (full sky) report discoveries at $5 \sigma$ significance in less than one year. The biggest scientific challenge in the newborn HE neutrino astronomy, is of having a combined single distributed planetary instrument, known as HE PLanEtary neutrino Monitoring system (PLEvM), an United Nations Open Universe initiative [15]. The Latin American community is ready to join the global efforts, either on motivations/experience on $\mathrm{HE}$ astrophysical data analysis, as well as in construction and commissioning of observatories, and flux predictions (Table 5.3).

Additionally, as the multi-purpose capability of $\mathrm{KM} 3 \mathrm{NeT}$ for neutrino parameters studies, ORCA, with atmospheric neutrinos as beam, will provide a $3 \sigma$ sensitivity on $\mathrm{NH}$ after three years of exposure and competitive precision in $\Delta m_{32}^{2}(2-3 \%)$ and $\sin ^{2} \theta_{23}(4-10 \%)$. See section 5.2 .1 for a detailed description of a new proposal for a facility in Latina America focused on $v_{\tau}$ astronomy.

\subsubsection{Search for new neutrinos states: light sterile neutrinos and heavy neutral leptons}

The study of neutrino oscillations is based on the $3 v$ paradigm suggested by the structure of the Standard Model (SM), namely the existence of three complete generations. This means that whether right-handed neutrinos exist or not, there are only three light species that oscillate among themselves. This is of course the minimal possibility and additional sterile neutrino states could exist that would in principle oscillate with the three usual states, leading to a richer oscillation parameter space. In fact, the anomalies that have been observed in experiments at short-baseline accelerators, reactors, and source (Gallium) (LSND, MiniBooNE, Gallex, SAGE) can not be accounted for by the $3 v$ paradigm and sterile neutrinos have been proposed as a possible remedy. It turns out, however, that even with several additional sterile neutrinos, it is not possible to explain all available data [16] [17]. The existence of these anomalies has motivated several reactor- and acceleratorbased experiments such as the NEutrino Oscillation at Short baseline (NEOS) in South Korea, the Search for Sterile Reactor Neutrino Oscillations (STEREO) in France, Neutrino-4 in Russia and the Detector of AntiNeutrino based on Solid Scintillator (DANSS) in Russia, Precision Reactor Oscillation and Spectrum Experiment (PROSPECT) in the USA, the Search for short baseline antineutrino disappearance with composite Lithium-6 scintillator detectors (SoLiD) in Belgium, experiments ID137 at Fermilab, the MicroBooNE experiment (BooNE - Booster Neutrino Experiment) that uses the same neutrino beam as MiniBooNE and that involve a future phase with the implementation of the Short-Baseline Near Detector (SBND) and Imaging Cosmic And Rare Underground Signals (ICARUS) in the near future. Also DUNE contains a program for the study of sterileactive oscillation parameters.

The potential existence of sterile states brings about the possibility for the presence of heavy neutral leptons that could be produced in colliders. In fact, from the theoretical and model-building point of view, these states are common in most extensions of the SM, and in some cases predicted/expected to exist with a mass scale close to the electroweak scale, thus available for experimental searches. Such searches have been performed at existing colliders. Sensitivity studies have been made for proposed experiments such as FASER, MATHUSLA and CODEX-b, and for future colliders (e.g., the FCC) as well. For some mass scales of the heavy neutral leptons it could be also detected in neutrino experiments such DUNE.

It is clear from this that there is a community-wide interest in the study and potential discovery of these states, namely sterile neutrinos and heavy neutral leptons, whose motivation comes from the study of Beyond Standard Model (BSM) physics and Non-Standard Interactions (NSI). 


\subsection{Research infrastructures}

The diversity of neutrino experiments are providing valuable results to fit the global neutrino picture. Furthermore, underground/ice/water observatories offer excellent conditions for multidisciplinary research in Earth and Life Sciences as geophysics and biology (such as the proposed ANDES laboratory, see below), as well as in subatomic sciences as searches for rare events (e.g., $\beta \beta 0 v$, proton decay), exotic physics (e.g. DM), tests of general relativity, etc. They also offer low background conditions for atmospheric, reactor, solar (spacebased accelerators in general), and earth-based accelerator neutrino experiments [18] [19]. Additionally, nuclear astrophysics processes, involving neutrinos as a probe are also favored.

Neutrinos appear in the Latin America context in sync with global efforts to address the open questions of Neutrino S\&T. Latin American countries currently involved in neutrino experiments either international and local collaborations are shown in Figure 5.3

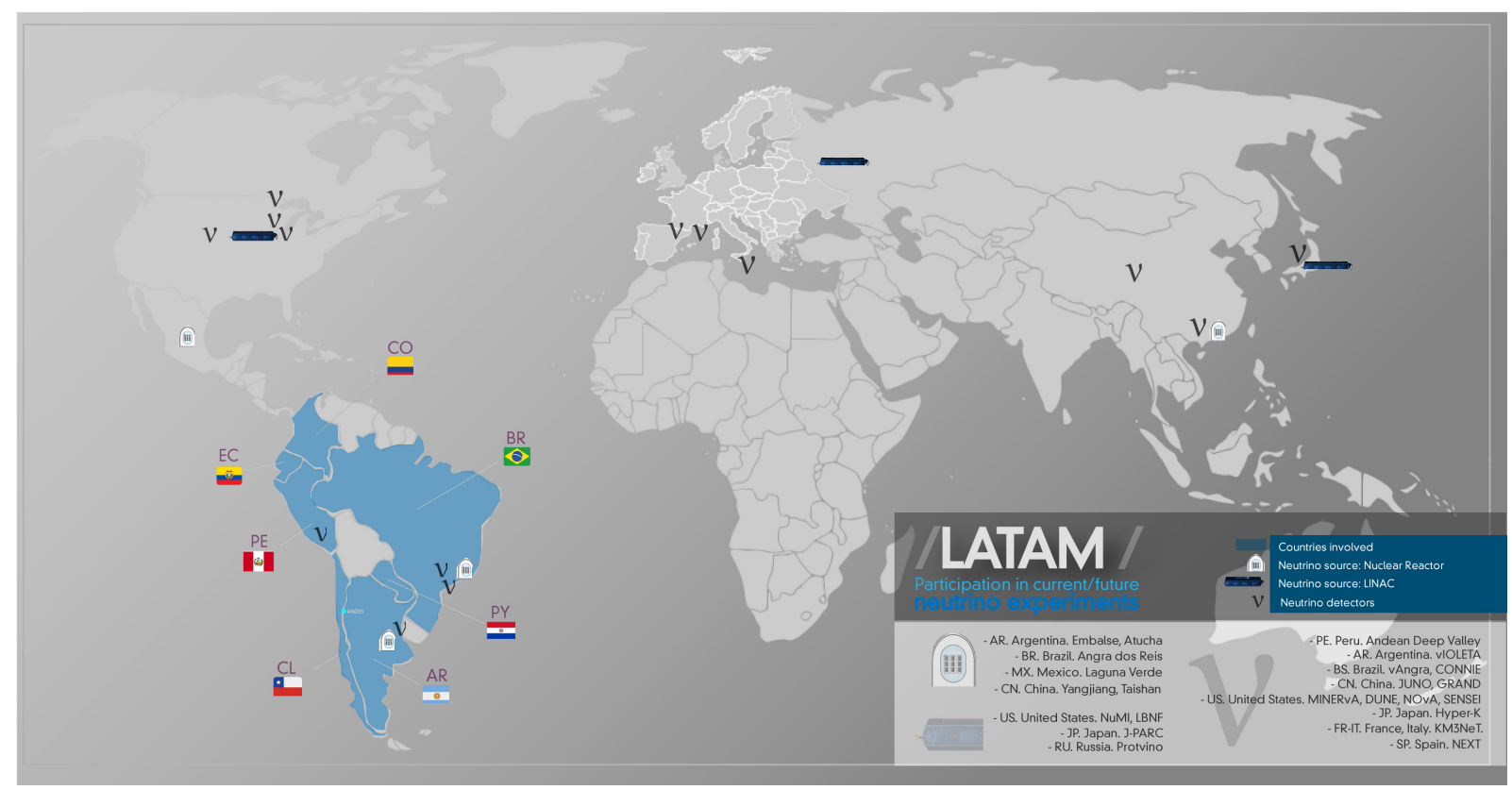

Figure 5.3: Latin American participation in current/future neutrino experiments: earth-based neutrino sources and detectors are indicated, space-based neutrino sources are assumed by default. MINERvA, whose data period has ended, is still providing world's best high-precision neutrino cross-section measurements, is also represented.

Currently, Latin America has a strong involvement in the US in neutrino experiments such as DUNE, mainly featured by the design and construction of the Photon Detection System (PDS) for the single phase far detector modules. The PDS consists of a light-trap concept known as ARAPUCA or its variant X-ARAPUCA and its corresponding readout systems [20]. Other important participation of Latin American groups has been in the Minerva, Nova and SBND projects. Latin America is also involved in specific programs in EU with CERN and other large-scale multidisciplinary research infrastructures as $\mathrm{KM} 3 \mathrm{NeT}$, setting a promising versatile future for Latin America in Neutrino S\&T global effort.

The feasibility of the ANDES laboratory in South America [21] becomes very strategic towards the future of the neutrino community in Latin America as a flagship local large-scale infrastructure, run by an international consortium. ANDES is aimed to be installed in an international tunnel ( $\sim 45 \mathrm{~km}$ long) to be constructed (20212029) along the Agua Negra Pass between Chile (Coquimbo) and Argentina (San Juan). ANDES will consist of a Main Hall $(21 \mathrm{~m} \times 23 \mathrm{~m} \times 50 \mathrm{~m})$, Secondary Hall $(16 \mathrm{~m} \times 14 \mathrm{~m} \times 40 \mathrm{~m})$, the Large Pit $(30 \mathrm{~m}$ diameter, 30 $\mathrm{m}$ height), a geoscience area, offices and small labs. The vertical depth of ANDES is $\sim 1.8 \mathrm{~km}$, omnidirectional $\sim 1.7 \mathrm{~km}$, with horizontal road access, in total the facility reaches $70 \mathrm{k} \mathrm{m}^{3}$ of laboratory volume plus $35 \mathrm{k} \mathrm{m}^{3}$ access tunnels. The Large Pit, can be used to place a next generation neutrino detector, the whole volume of the pit would be filled with ultra-pure water as shielding, waterproof against any leak coming from water of the rocks forming the cavern. The Main Hall could be available for hosting another emblematic experiment, e.g., 
a $\beta \beta 0 v$ detector. ANDES may host neutrino experiments with a complementary global science programs, e.g., neutrino nature, cosmic neutrinos, DM neutrinos, Geoneutrinos, NSI, and applications in homeland security as Far Field (FF) site [21], see also [1-32]. A Supernovae neutrino program in ANDES would allow coupling to Global Neutrino Network (GNN) [22] and SuperNova Early Warning System (SNEWS) [23], and follow-up under a joint sub-threshold analysis of offline time/space correlation multi-messenger searches (e.g., KM3NeT, CTA, LSST, SKA, HAWC, SVOM, LIGO/VIRGO, TAROT, ELT) some, with already relevant Latin America participation.

Globally, the widely explored technologies in neutrino detection can be summarized as: a) Scintillation (noble elements gas, liquid, solid or dual phase) and Hydrid Detectors (e.g., Scintillation + Cherenkov), b) WCDs (liquid, solid/ice), c) Radio Cherenkov Detectors (ice, atmosphere) and Pressure-Waves Acoustic Detectors (water, ice), and d) Pixelated Detectors (semiconductors). The light/charge readout as a result of the light/charge response of the detection medium ( $\mathrm{LAr}, \mathrm{GXe}, \mathrm{C}_{x} \mathrm{H}_{y}$ compounds, sea water, purified water, ice, salt, air, etc.), is usually performed by a synchronized performance of SiPMs, PMTs, WaveLength Shifter (WLS), Front End Boards (FEBs) and Data AcQuisitions systems (DAQ), etc.

The NEXT experiment at the Canfranc Underground Laboratory (LSC), with Colombian participation, is a HighPressure Gaseous Xenon (HPGXe) TPC with independent planes for energy (PMTs) and tracking (SiPMs), a good example of hybrid detection system [24].

The next subsections present details of neutrino experiments with participation of Latin American groups that submitted a white paper during the LASF4RI-HECAP process.

\subsubsection{Latin America-based large-scale infrastructures}

TAMBO: Tau Air Shower Mountain-Based Observatory This is a proposal to be implemented in the Peruvian Colca Valley at the Tau Air shower Mountain-Based Observatory (TAMBO), as an array of small WCDs $\left(\sim 22 \mathrm{k}, 1 \mathrm{~m}^{3}\right.$ each, spaced by $100 \mathrm{~m}$ on the mountain slope), for HE $v_{\tau}$-astronomy with high background suppression [25]. Andean Deep-Valley science objectives span: study of HE neutrino sources above 10 $\mathrm{PeV}$, characterization of sources between 1-10 PeV range by measuring the tau component, and to constrain the particle acceleration potential of point source transients observed via multi-messenger astronomy. The $v_{\tau}$ propagates in rock with an interaction length of thousands of kilometers for the energy range of interest, it releases $\tau$ s by Charged-Current (CC) interactions (range $\sim 50 \mathrm{~m}-5 \mathrm{~km}$ before decay) transferring $\sim 80 \%$ of its energy to the medium. If the decay happens within this range, it will be knocked out from rock to air and may indeed decay in air and about $50 \%$ of its energy goes into particles that produce Extensive Air Showers (EAS). The longitudinal profile of the EM component of the EAS has a characteristic length of $\sim 10 \mathrm{~km}$ (mountains separations) and a diameter of $\sim 200 \mathrm{~m}$ near the EAS maximum. The topography where the array would be placed favours the geometric acceptance compared to a flat ground array. It is expected that Andean DeepValley will also shed light on discriminating photons from hadrons and contribute to $\gamma$-ray observations at the $\mathrm{PeV}$ range and beyond, and also to characterize the Cosmic Rays (CRs) anisotropy at PeV energies with new measurements at other latitudes. The TAMBO is close to producing a Conceptual Design Report, however, very interesting initiatives and a large collaborative effort are currently shaping up between Brazilian, Peruvian, US and EU counterparts. Andean Deep-Valley would be able to link to GNN and SNEWS mentioned above. Figure 5.4 shows the range of neutrino fluxes that would be probed by TAMBO.

\subsubsection{Latin America-based small-scale - high impact- infrastructures}

Neutrinos Angra Experiment ( $v$-Angra) The $v$-Angra collaboration has been running smoothly since 2018 a WCD located at Angra Dos Reis nuclear complex (core II), recording $\bar{v}_{e}$ events from Inverse Beta Decay (IBD) during fuel burnout looking to monitor the reactor dynamics [26]. About 354 gallons $\mathrm{GdCl}_{3}$ doped water $(0.2 \%)$ constitutes the radiator and detection medium, contained into $0.90 \mathrm{~m} \times 1.46 \mathrm{~m} \times 1.02 \mathrm{~m}$ (height-length-width) plastic tank surrounded by 32 eight-inches PMTs (16 top-placed, 16 bottom-placed), 6 internal faces covered by diffuse reflectors ( $99 \%$ reflectance at $400 \mathrm{~nm}$ ) and top veto with 4 PMTs strategically located for tagging pulses triggered by cosmic muon events. The readout electronics is made of conventional elements, after amplification and shaping by the FEB, digitization is performed by a VME-based crate system which gathers processes and outputs samples to a FPGA controlling the data stream. The dependence of the neutrino counting rate with the reactor's activity make neutrino detectors a remote monitoring tool of the reactor's activity, composition of burned fuel, thermal power production and targeting of plutonium (main 


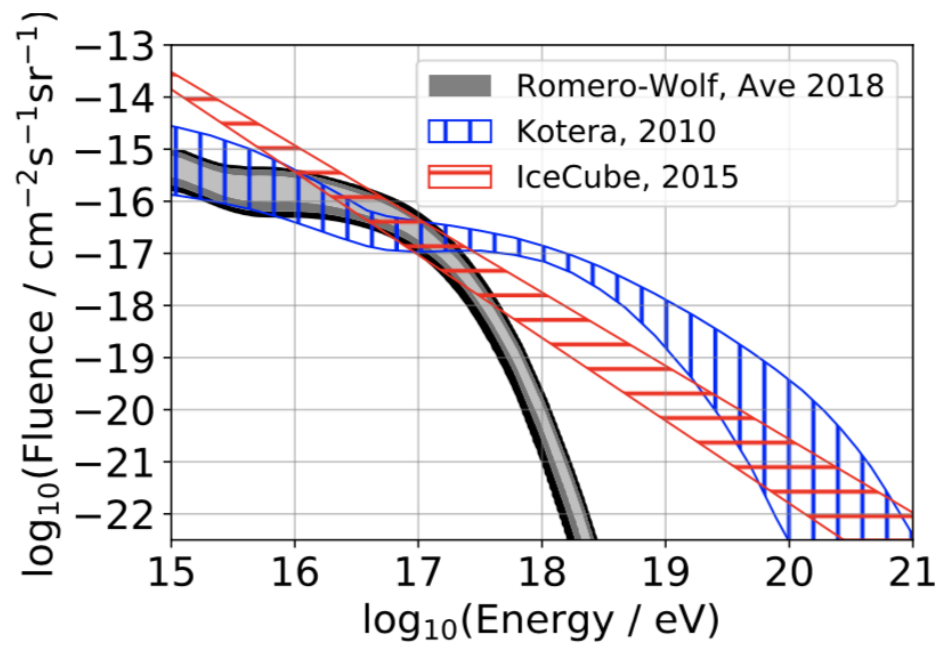

Figure 5.4: Neutrino fluxes probed by TAMBO, from 25].

threat-related issue). The $v$-Angra detector is located on the surface, $30 \mathrm{~m}$ away from the core, the optimal signal-to-background ratio is the biggest issue to overcome, in this sense the goodness on proximity to the core is as important as an optimized shielding (e.g., by overburden). Brazil, leading the $v$-Angra collaboration has successfully turned the experimental neutrino expertise into practical roles for the Latin America nuclear sector. This is a R\&D proposal looking for, in a midterm, be considered as an innovative tool in homeland security, synchronized to recognized US and EU efforts as WATCHMAN [27] and NUCIFER [28]. Such efforts must follow IAEA's protocols and motivations for FF reactor monitoring [29], as potential and novel technologies that can be applied for non-proliferation safeguards. Reliability and cost-effective technology (significantly lower if implemented in the operational budget of the plant) is the big challenge. Remote monitoring reduces dose in the operation, maintenance, verification and inspection staff, by skipping access to the containment building and other restricted areas.

Skipper-CCD experiments (CONNIE, SENSEI, $v$ IOLETA) These experiments with different physics motivations but same core technology (ultra-low threshold Skipper-Charge Coupled Device (CCD)) are Silicon pixelated detectors systems with a spatial resolution of $15 \mu \mathrm{m}$ capable of charge recording per pixel with a background noise RMS $\sim 0.1 e^{-}$(electrons individual counting). Skipper-CCDs were pioneered at Fermilab for the Sub-Electron-Noise Skipper Experimental Instrument (SENSEI) [30] searching for direct DM observation in the MeV-GeV range. Due to the ultra-low background conditions requirements for DM searches, SENSEI would be a good candidate to be host at ANDES, see chapter on dark matter for more details. Low-energy recoils from CEvNS (cross-section more than two orders of magnitude higher than in the neutrino-nucleon case) of reactor antineutrinos with Silicon nuclei and tests of NSI (including sterile neutrinos searches from anomalies in nuclear reactor SBL experiments) are the main physics goals inspired by this kind of technology. Coherent Elastic neutrino-Nucleus Scattering (CEvNS) can also be studied from solar, atmospheric and diffuse Supernovae neutrinos, relevant processes have been identified as background components for future DM searches and next generation experiments. CEvNS directly measured in controlled reactor experiments are useful for the parameterization and suppression of such components. The Skipper-CCD technology enables exploration of CEvNS at a SBL nuclear reactor experiment with CONNIE [31]. CONNIE is currently a detection array of 12 CCDs $(\sim 73 \mathrm{~g})$ running since 2016 at Angra-II in the same hut of $v$-Angra. Future upgrades consider Skipper-CCD technology $(\sim 10 \mathrm{~kg})$ for significant improvements in the lower energy threshold and sensitivity. Additionally, due to the ultra-low threshold feature of Skipper-CCDs, it is also a viable option for the quantification of $\bar{v}_{e}$ fluxes in experiments as $v$ IOLETA [32]. The latest proposal is for a multi-kilogram array placed at Atucha nuclear complex (core II), 8-12 $\mathrm{m}$ away from the core and inside the dome (concrete shielding against $\mathrm{CR}$ muons background and enhancement of $\overline{v_{e}}$ flux). Potential and flexibility of Skipper-CCD technology are reasonable criteria for boosting local cooperation between Argentina and Brazil and their impact 
on the nuclear industry, with the strategic partnership of Fermilab.

\subsubsection{International large-scale infrastructures}

Jiangmen Underground Neutrino Observatory (JUNO) is an underground liquid scintillator (Linear Alkyl Benzen) experiment of $\sim 20 \mathrm{kton}$ of total active mass under construction [4], located $53 \mathrm{~km}$ away from the Yangjiang (6 cores) and Taishan (2 cores) nuclear complexes in Southern China (Kaiping, Jiangmen). JUNO will work in antineutrino detection mode through IBD. The main focus of its neutrino physics programs spans: precision measurement of of $\theta_{12}$ and $\Delta \mathrm{m}_{21}^{2}$, NHM, Supernova and solar neutrinos, geo-neutrinos and proton decay. Institutions from Brazil and Chile are progressively getting involved in hardware through the sPMT ( 25k 3-inches small PMTs) subsystem (Under Water Box and HV-Splitter), software (simulation of ABC FEB) and physics (solar oscillation parameters, physics potential). JUNO is the most representative reactor-neutrino experiment nowadays, continuing the remarkable legacy of experiments as Daya Bay, Double Chooz and RENO.

Deep Underground Neutrino Experiment (DUNE) This experiment is under construction formed by a Near Detector (ND) (Fermilab) and a Far Detector (FD) located at Sanford Underground Research Facility (SURF), spaced by $1300 \mathrm{~km}$ and exposed along the LBNF beam line 20]. DUNE is designed to address precision measurements of $\theta_{23}$ octant, $\mathrm{NMH}$ and potential discovery of $\mathrm{CP}$ violation in leptons, $\delta_{C P}$. The FD (charge and light detection, calorimetry-positioning, light-yield field) is a LAr TPC of $\sim 40 \mathrm{k}$ tons of total active mass equally segmented into four modules. Scintillation light from interactions in LAr $(\sim 40$ photons $/ k e V$ when excited by a minimum ionizing particle, $127 \pm 10 \mathrm{~nm}$, WLS used) plays a major role in the timing, triggering and calorimetry of the detector. Brazil is strongly connected to DUNE efforts with major involvement in all the strategic areas: hardware (PDS of FD, with the ARAPUCA detector conceived, designed and developed in Brazil, see chapter on Instrumentation and Computing for more details), software (Monte Carlo simulations for light production and propagation) and physics (BSM and Supernova Neutrinos). Colombian, Peruvian and Paraguayan groups are mainly involved in the hardware of the PDS of DUNE, specifically through the digitization boards for SiPMs (required to run at room temperature) for the main readout electronics system so-called "DAPHNE". Colombian groups' interest in physics are connected to NSI and DM. The Peruvian groups are also contributing releasing phenomenological analyses of DUNE sensitivity to Beyond Standard Model phenomena [33].

NuMI Off-axis $v_{e}$ Appearance experiment (NOvA) An inspiring experiment with valuable operational experience for DUNE. NOvA has a $810 \mathrm{~km}$ baseline (Fermilab to Minnesota), formed by identical two neutrino detectors (ND of $\sim 300$ metric-ton $1 \mathrm{~km}$ from NuMI beam and FD of $\sim 14$ metric-kiloton), both are made up of $344 \mathrm{k}$ cells of plastic PVC filled with liquid scintillator $\left(\mathrm{C}_{9} \mathrm{H}_{12}\right.$ pseudocumene + special additives, with mineral oil as solvent) [34]. Neutrino-scintillator interactions release a burst of charged particles whose energy is collected using WLS connected to Avalanche PhotoDiode (APD) arrays for readout. NOvA physics programs are focused on the precise determination of $\theta_{23}$ and $\Delta \mathrm{m}_{32}^{2}, \mathrm{NMH}$ and $\delta_{C P}$. Colombian contributions are in physics analysis: cross-section data analysis in neutrino or antineutrino-mode (inclusive and exclusive channels) and reduction of uncertainties: axial masses, final state interactions, random phase approximation and the $2 \mathrm{p} 2 \mathrm{~h}$.

Short Baseline Neutrino Experiment (SBND) In addition to the experimental observation of the three neutrino picture described above, several experimental "anomalies" have been reported which, if experimentally confirmed, could be hinting at the presence of additional neutrino states with larger mass- squared differences participating in the mixing. SBDN is one of the three detectors part of the Short Baseline neutrino program at Fermilab, where neutrino or anti-neutrino beams can be produced in the same experiment and, both charged current and neutral-current channels can be explored to clarify these anomalies. The Brazilian group involved in DUNE is also participating in SBND with an R\&D program for novel photon detection systems.

KM3 Neutrino Telescope (KM3NeT) It is an international collaboration constructing the future $\mathrm{km}^{3}$-scale deep-sea neutrino observatory in two abyssal sites at the Mediterranean: ARCA, near Capo Passero in Sicily (Italy), optimized for cosmic and astrophysical neutrinos, and ORCA, near Toulon (France), optimized for 
atmospheric neutrinos [35]. ARCA and ORCA, as multi-purpose neutrino detectors, share the same technology but their layouts are sparser and denser respectively. KM3NeT scaling is in progress aiming to complete 3 building blocks (2-ARCA, 1-ORCA) formed by 115 Detection Units (DUs) each comprising of 18 Digital Optical Modules (DOMs) (31 three-inches PMTs, calibration systems, central logic board and power supply) vertically arranged and equally spaced. The Cherenkov light induced by relativistic charged leptons produced by CC neutrino interactions when crossing the detection media is collected by the DUs array. KM3NeT owns unique features as a multi-disciplinary infrastructure for Life and Earth Sciences: permanent connection, real time, and large band-width for undersea communications with shore stations on the coast or in remote operation. User ports available in the submarine network of the seabed allows to connect specialized instrumentation and modules operating in challenging environments. Main physics programs search for with ARCA for the discovery and subsequent study of extraterrestrial neutrino sources, while with ORCA, the aim is to measure $\theta_{23}$ and $\Delta \mathrm{m}_{32}^{2}$, and $\mathrm{NMH}$ with atmospheric neutrino sources. In addition searches for to NSI, exotic physics (monopoles, nuclearities), test of fundamental physics and $v$-tomography (attenuation and oscillation) are also part of the physics program. The P2O proposal, a Long Base Line $(\mathrm{LBL})$ experiment of $\sim 2600 \mathrm{~km}$, aiming to shoot a neutrino beam from Protvino tovthe ORCA site is the bet towards a measurement of $\delta_{C P}$. The dense core of ORCA offers in turn lots of possibilities for indirect DM searches, and would be open for a strong and strategic collaboration with Latin America. Current activities in Ecuador are focused on hardware (calibration) both for the photonics (DOM angular acceptance, HV tuning of light sources) and acoustic positioning subsystems (digital compasses), as well in the design of the OCS. Regarding physics contribution to Blazars or Supernovae neutrinos is expected to start soon.

Hyper-Kamiokande (Hyper-K) The next generation of the Super-K detector is known as Hyper-K [36], it will continue the legacy of the successful operative experience and challenging physics programs. In this version, two new WCDs are considered: a $0.5 \mathrm{kt} \mathrm{ND}$ and 258(188) kton FD of total(fiducial) mass, at $\sim 1 \mathrm{~km}$ and $295 \mathrm{~km}$ from the Japan Proton Accelerator Research Complex (J-PARC) neutrino beam, respectively. The design of Hyper-K, consists of drum-shaped tanks of $10 \mathrm{~m}$ in diameter $\times 8 \mathrm{~m}$ in height for the ND, and $60 \mathrm{~m}$ in diameter $\times 74 \mathrm{~m}$ in height for the FD. The photosensor arrays are $\sim 500$ multi-PMT(19) DOMs in the ND in charge of measuring flux and cross-section in order to reduce systematics at FD, and 40k twenty-inches inner detector PMTs (first option) or 50\% twenty-inches + multi-PMT DOM, in addition $\sim 7 \mathrm{k}$ outer veto detector PMTs at the FD. The tanks will be filled with ultra-pure water ( $\sim 260 \mathrm{k}$ tons in the FD) as radiator media for collecting signals coming from CC neutrino interactions with the electrons of nuclei of water, relativistic electrons or positrons creating the distinguished Cherenkov rings. Hyper-K will be located under the Nijugo mountain $295 \mathrm{~km}$ away from the J-PARC neutrino beam facility in Tokai, Japan. The main physics goals of Hyper-K span from determination of $\theta_{23}, \Delta \mathrm{m}_{32}^{2}$ and precision measurements of oscillation parameters, and $\mathrm{NMH}$ supported by atmospheric and J-PARC neutrino sources, $\delta_{C P}$ observation with J-PARC neutrinos, Supernova and relic neutrinos, and ${ }^{8} \mathrm{~B}$ neutrinos from Sun's fusion reactions, proton decay and DM searches, and other exotic phenomena as sterile neutrinos and tests of Lorentz invariance. Brazil is currently involved in Hyper- $\mathrm{K}$ in physics studies of NSI, as well as possible contributions to GRID computing and data storage.

Major physics drivers: $\theta_{23}, \Delta \mathrm{m}_{32}^{2}$, precisions, $\mathrm{NMH}$,

Giant Radio Array for Neutrino Detection (GRAND) The GRAND's concept is based on a large array of antennas (20 subarrays with $10 \mathrm{k}$ antennas each over $\sim 10 \mathrm{k} \mathrm{km}^{2}$ ) for detection of radio emission from EAS triggered by cosmic-ray interactions with atmosphere [37]. The detection array consists of $5 \mathrm{~m}$-high antennas optimized for horizontal showers bow-tie design, 3 perpendicular arms, frequency range $50-200 \mathrm{MHz}$, with a inter-antenna spacing of $1 \mathrm{~km}$. GRANDProto300 prototype would be suited with 300 detection units (radio antennas + surface detectors) over $\sim 200 \mathrm{~km}^{2}$ with denser infill array in a prospective site in QingHai province, China. GRAND's primary goals are focused on Ultra-High Energy (UHE) neutrinos (cosmogenic and astrophysical), with secondary goals in cosmology and radioastronomy. GRANDProto300 will be able to study the transition between galactic and extra-galactic CRs. GRAND10k constitutes an intermediate step in GRANDS's timeline. Once its final layout, GRAND200k, the observatory would be able to detect cosmogenic neutrinos (produced by UHE CRs interactions while intergalactic propagation) with energies larger than $100 \mathrm{PeV}$ by radio signal detection produced by the interaction of UHE CRs, $\gamma$-rays and neutrinos with Earth's atmosphere. Brazil and Argentina are currently involved in GRAND collaboration (planned), and their roles are still an open issue. GRAND will be able to link to GNN and SNEWS mentioned above, and couple to a multi-messenger strategy with Latin America participation. 
Table 5.3: Potential capabilities and involvement in neutrino science by the Latin American community: sources, phenomenology and theory SI/NSI. Based on historical collaborative records and current LASF4RI-HECAP process. [38].

\begin{tabular}{cccccccccc}
\hline Country & Geo LE & Cosmic ME & Cosmic HE & SI (XS, CEvNS) & Masses & $\theta_{23}$ & NMH & $\delta_{C P}$ & BSM, NSI (e.g., DM) \\
\hline CO & - & - & - & $\sqrt{ }$ & $\sqrt{ }$ & $\sqrt{ }$ & $\sqrt{ }$ & $\sqrt{ }$ & $\sqrt{ }$ \\
PE & - & - & - & $\sqrt{ }$ & - & - & - & - & $\sqrt{ }$ \\
PY & - & - & - & $\sqrt{ }$ & - & - & - & - & $\sqrt{ }$ \\
EC & - & $\sqrt{ }$ & $\sqrt{ }$ & - & - & $\sqrt{ }$ & $\sqrt{ }$ & $\sqrt{ }$ & $\sqrt{ }$ \\
AR & - & $\sqrt{ }$ & $\sqrt{ }$ & $\sqrt{ }$ & - & - & - & - & $\sqrt{ }$ \\
BR & $\sqrt{ }$ & $\sqrt{ }$ & $\sqrt{ }$ & $\sqrt{ }$ & - & $\sqrt{ }$ & $\sqrt{ }$ & $\sqrt{ }$ & $\sqrt{ }$ \\
CL & $\sqrt{ }$ & $\sqrt{ }$ & - & - & - & - & $\sqrt{ }$ & - & $\sqrt{ }$ \\
\hline
\end{tabular}

Table 5.4: Potential capacities and involvement in neutrino technology by the Latin American community: detector instrumentation and related technologies. Based on historical collaborative records and current LASF4RIHECAP process [38].

\begin{tabular}{cccccccc}
\hline Country & SiPMs & PMTs & CCDs & Acoustic/Radio & FEB/DAQ & Protocols (EO/OE, WR) & Al, IT, GRIDs \\
\hline CO & $\sqrt{ }$ & $\sqrt{ }$ & - & - & - & $\sqrt{ }$ & $\sqrt{ }$ \\
PE & $\sqrt{ }$ & $\sqrt{ }$ & - & - & $\sqrt{ }$ & - & $\sqrt{ }$ \\
PY & $\sqrt{ }$ & - & $\sqrt{ }$ & - & $\sqrt{ }$ & - & - \\
EC & - & $\sqrt{ }$ & - & - & - & - & $\sqrt{ }$ \\
AR & - & - & $\sqrt{ }$ & - & - & - & - \\
BR & $\sqrt{ }$ & $\sqrt{ }$ & $\sqrt{ }$ & $\sqrt{ }$ & $\sqrt{ }$ & $\sqrt{ }$ & $\sqrt{ }$ \\
CL & - & $\sqrt{ }$ & - & - & - & - & - \\
\hline
\end{tabular}

\subsection{Areas of excellence in Latin America}

There are seven countries actively engaged in neutrino projects in the Latin American region: Colombia (CO), Peru (PE), Paraguay (PY), Ecuador (EC), Argentina (AR), Brazil (BR) and Chile (CH) 38. The identified capacities in theory and phenomenology, and from participation in neutrino experiments as presented in Section 5.2 is summarized in Table 5.3

Columns 2-4 refers to potential capabilities and experience in neutrino observatories for geo and cosmic neutrinos studies, 5-6 for Standard Interactions (SI) and absolute neutrinos masses, 7-9 for still unknown parameters, and last column for open opportunities in NSI and advanced physics programs. On the other hand, Table 5.4 shows identified capacity building in detectors instrumentation and related technology.

In this case, columns 2-5 stand for photonics and acoustics experience in neutrino detection systems and calibration, 6-7 for readout, DAQ and communications, last column for declared Data Science experience, Information Technology, and computing management and availability in neutrino projects. With respect to the last item, several highly-qualified scientists and engineers from Venezuela, Puerto Rico and Mexico with a lot of experience running HEP projects [39] locally and internationally could contribute to these efforts.

\subsection{Synergies}

\subsubsection{Local large-scale infrastructures}

ANDES and TAMBO are two of the future initiatives for a large-scale implementation in the region. The construction of ANDES, supported by the international community, would hopefully be starting in a 5-10 years timeline. Once civil constructions is finalized it could host two neutrino experiments in the Main Hall and the Large Pit. A Supernovae neutrinos experiment might be an option for the Main Hall. The Large Pit could host a a $\beta \beta 0 v$ neutrino experiment, as this can be a valuable opportunity for Latin America (Table 5.3). On 
the other hand, TAMBO is also as a very good option for $v_{\tau}$-astronomy, being a groundbreaking field, globally attractive and being already supported by US and EU counterparts. TAMBO does not use "new technologies", the detection array concept is based on well known WCDs incorporating PMTs with nanosecond time resolution is commercially available, with electronics relatively simple to use (e.g., AUGER, LAGO). WCDs overlap with other HECAP experiments such as HAWC and SWGO also supported by Latin America collaborators with complementary roles. Very-large volume neutrino observatories as KM3NeT are suited for making their science programs looking $v_{\mu}$ as the golden channel, multiOmessenger programs are therefore another strategic factor for Latin American. Thanks to IceCube's breakthrough discoveries and plans for upgrades, the scaling of KM3NeT and the arrival of new neutrino observatories (e.g., GVD, P-ONE) will make possible to survey the whole sky with unprecedented potential for discovery and increased sensitivity.

\subsubsection{Local small-scale infrastructures}

The pair of Skipper-CCD experiments $v$ IOLETA and CONNIE are intimately connected not only by the potential offered by the technology, but by the practical roles of applied antineutrino technology. These outstanding short-scale Near Field (NF)-SBL projects, may garner support and for the upgrade of the experiments and development of a FF-LBL option. Some proposals have been discussed in the past in national meetings with funding agencies and stakeholders, where the construction of the FF site is the big challenge. The bet for FF sites for $v$ IOLETA, CONNIE, $v$-Angra, and detector upgrades may sound reasonable. Several prototypes during R\&D might indeed be early tested in the several research reactor facilities in Brazil and Argentina. Moreover, links between governments, funding agencies and international organizations such as IAEA are supporting the progress of this kind of technology through detailed technical cooperation programs and more.

\subsubsection{International large-scale infrastructures}

The participation in many international collaborations covering almost all cutting-edge neutrino physics programs have allowed important advances in training, networking and qualified human resources. The Latin American strategy must also recognize and value current collaborations mainly started by huge individual efforts. In this sense, initiatives as discussed in Subsections 5.4.15.4.2 complete the picture of prioritized international multidisciplinary large-scale infrastructures, multi-purpose neutrino facilities and common core technologies.

In order to facilitate make sustainable and stronger the participation in these international efforts support is needed for local labs and computing centers, funding for mobility, graduate programs, contributions to largescale experiment common funds for ensuring construction and operative overheads. Concretely, in terms of hardware, Latin America commitments are in: a) commissioning of the PDS of DUNE, b) commissioning of the OCS of KM3NeT, c) commissioning of the SPMT subsystem of JUNO. Physics, data analysis and highperformance computing tools are naturally open to all collaborative efforts, implying budget significantly lower, but equally important contributions, than in hardware. Strong theory groups are spread throughout Latin America, participating e.g. in NOvA and Hyper-K, with vast experience on neutrino physics.

\subsection{Conclusions}

Beyond physics, multi-disciplinary and multi-purpose drivers are strategic in the synchronized and visionary efforts of HECAP on a global scale. KM3NeT is an example with permanent connection, real time, and large band-width for undersea communications with shore stations, and unprecedented experimental conditions for Earth and Life Sciences. In addition, it is making possible neutrino physics with atmospheric, cosmic and astrophysical sources. TAMBO and ANDES are two proposed local large-scale initiatives. The future ANDES laboratory would also offer multi-disciplinary and/or multi-purpose science opportunities. The Angra Dos Reis neutrino lab fits this criteria as well providing options for applied neutrino physics prototypes as $v$ Angra (nuclear safeguards) and basic science with CONNIE (coherent elastic neutrino-nucleus scattering), with remarkable testing of novel technologies. This also applies to $v$ IOLETA, a strategic facility in the Latin America roadmap. The pair of Skipper-CCD experiments $v$ IOLETA and CONNIE are in turn intimately connected and important in the strategy for local short-scale infrastructures. 
Regarding international large-scale infrastructures we note that the PDS system of DUNE, the OCS of KM3NeT, the sPMT of JUNO, have been identified as important initiatives in the region. 


\section{Bibliography}

[1] Carlo Giunti and Thierry Lasserre, eV-Scale Sterile Neutrinos, Annual Review of Nuclear and Particle Science, 69, 163-190, (2019)

[2] P.A. Zyla et al. (Particle Data Group), Neutrino Mixing - Particle Data Group, Prog. Theor. Exp. Phys., 083C01, page numbers, 1 June (2020)

[3] C. Giganti, S. Lavignac, M. Zito, Neutrino oscillations: The rise of the PMNS paradigm, Prog.Part.Nucl.Phys., 98, 1-54, January (2018)

[4] Yu-Feng Li, Jun Cao, Yifang Wang, and Liang Zhan, Unambiguous determination of the neutrino mass hierarchy using reactor neutrinos, Phys. Rev. D, 88, 013008, 16 July (2013)

[5] Ivan Esteban, M.C. Gonzalez-Garcia, Michele Maltoni, Thomas Schwetz, Albert Zhou, The fate of hints: updated global analysis of three-flavor neutrino oscillations, arXiv.org Repository, 2007.14792, 1-16, 27 July (2020)

[6] Ivan Esteban et. al, Nufit5.0 (2020), nufit webpage, http://www.nu-fit.org

[7] The DUNE Collaboration, Deep Underground Neutrino Experiment (DUNE), Far Detector Technical Design Report, Volume I, arXiv.org Repository FERMILAB-PUB-20-024-ND, 2002.02967v1 Volume I Introduction to DUNE, 1-244, 7 February (2020)

[8] D. Zaborov et. al., Letter of interest for a neutrino beam from Protvino to KM3NeT/ORCA, Eur. Phys. J. C, 79:758, page numbers, 12 September (2019)

[9] Nature volume 580, pages339-344 (2020)

[10] International Atomic Energy Agency (IAEA), Nuclear Power Reactors in the World, REFERENCE DATA SERIES No 2, IAEA-RDS-2/40, ISBN 978-92-0-114820-9, ISSN 1011-2642, 1-86, (2020)

[11] L. Cardani, Neutrinoless Double Beta Decay Overview, SciPost Phys. Proc. 1, 024, 1-15, 20 February (2019)

[12] P.A. Zyla et al. (Particle Data Group), Prog. Theor. Exp. Phys. 2020, $083 C 01$ (2020)

[13] IceCube Collaboration, Neutrino emission from the direction of the blazar TXS 0506+056 prior to the IceCube-170922A alert, Science, 361 Issue 6398, 147-151, 13 July (2018)

[14] The KM3NeT Collaboration, S. Aiello, et al., Sensitivity of the KM3NeT/ARCA neutrino telescope to point-like neutrino sources, Astroparticle Physics (111), 100-110, (2019)

[15] Elisa Resconi \& ECP team, UofA, ONC-UofVic, PLEvM - Towards a Planetary Neutrino Monitoring System, Talk at XVIII International Workshop on Neutrino Telescopes, Venezia, 18-22 March (2019)

[16] M. Dentler, Á. Hernández-Cabezudo, J. Kopp, P. A. N. Machado, M. Maltoni, I. Martinez-Soler and T. Schwetz, JHEP 08, 010 (2018) doi:10.1007/JHEP08(2018)010 [arXiv:1803.10661 [hep-ph]].

[17] S. Gariazzo, C. Giunti, M. Laveder and Y. F. Li, JHEP 06, 135 (2017) doi:10.1007/JHEP06(2017)135 [arXiv:1703.00860 [hep-ph]].

[18] Alessandro Bettini, New underground laboratories: Europe, Asia and the Americas, Physics of the Dark Universe, 4, 36-40, (2014)

[19] Aldo lanni, Considerations on Underground Laboratories, Journal of Physics: Conference Series, 1342 012003, 1-7 (2020)

[20] The DUNE Collaboration, Deep Underground Neutrino Experiment (DUNE), Far Detector Technical Design Report, Volume IV, arXiv.org Repository FERMILAB-PUB-20-027-ND, 2002.03010v1 Volume IV DUNE Far Detector Single-phase Technology, 1-672, 7 February (2020)

[21] X. Bertou, The ANDES Deep Underground Laboratory in South America: status and prospects (Y7.00001), APS April Meeting 2017, Session Y7: Deep Science Around the World, 1-39, 31 January (2017) 
[22] The Global Neutrino Network (GNN), https://www.globalneutrinonetwork.org/

[23] SNEWS: SuperNova Early Warning System, https://snews.bnl.gov/

[24] The NEXT Collaboration (F. Monrabal et al.), The Next White (NEW) Detector, J. Inst., 13 No 12, P12010, 6 December (2018)

[25] Andres Romero-Wolf et. al., An Andean Deep-Valley Detector for HighEnergy Tau Neutrinos, arXiv.org Repository, 2002.06475v1, 1-16, 16 February (2020)

[26] H.P. Lima Jr. et. al., Neutrinos Angra experiment: commissioning and first operational measurements, J. Inst., 14 P06010, 1-16, 5 June (2019)

[27] Viacheslav Li, Far-Field Monitoring of Reactor Antineutrinos for Nonproliferation, arXiv.org Repository, 1907.08891v1, 1-12, 21 July (2019)

[28] G. Boireau et al. (Nucifer Collaboration), Online Monitoring of the Osiris Reactor with the Nucifer Neutrino Detector, Phys. Rev. D, 93, 112006, 10 June (2016)

[29] International Atomic Energy Agency (IAEA), Focused Workshop on Antineutrino Detection for Safeguards Applications, IAEA Report, STR-361, 1-39, October (2008)

[30] Javier Tiffenberg, The SENSEI Experiment, Astrophys.Space Sci.Proc., 56, 137-142, November (2019)

[31] Alexis Aguilar-Arevalo et al. (CONNIE Collaboration), Exploring low-energy neutrino physics with the Coherent Neutrino Nucleus Interaction Experiment, Phys. Rev. D, 100, 092005, 13 November (2019)

[32] Guillermo Fernandez Moroni on behalf of $v$ IOLETA, Short baseline neutrino experiment in nuclear reactors using Skipper CCD, Talk at II Latin American Strategy Forum for Research Infrastructure: an Open Symposium for HECAP, Day II: Neutrinos, 4 July (2020)

[33] Díaz, F. N. and Hoefken, J. and Gago, A. M, Phys. Rev. D, 102:055020, 2020, Carpio, J. A. and Massoni, E. and Gago, A. M., Phys. Rev. D, 100:015035, 2019. Carrasco, J. C. and Díaz, F. N. and Gago, A. M, Phys. Rev. D 99:075022, 2019.

[34] Tomas Nosek, NOvA Recent Results with Neutrino + Antineutrino Data, New Trends in High-Energy Physics 2018: Proceedings of the Conference, arXiv.org Repository 1905.09109v1, 1-10, (2019)

[35] S. Adrián-Martínez et al., KM3NeT 2.0 - Letter of Intent for ARCA and ORCA, J. Phys. G: Nucl. Part. Phys., 43, 084001, 24 June (2016)

[36] Y. Kudenko, Hyper-Kamiokande, J. Inst., 15, C07029, July (2020)

[37] Álvarez-Muñiz, J., Alves Batista, R., Balagopal V., A. et al., The Giant Radio Array for Neutrino Detection (GRAND): Science and design, Sci. China Phys. Mech. Astron., 63, 219501, (2020)

[38] E. Kemp, H. Yepes-Ramirez, H. Nunokawa, A. Romero-Wolf, I. Nasteva, D. Moreno, P. Chimenti, G. Moroni, X. Bertou, E. Segreto, R. Batista, Talks at II Latin American Strategy Forum for Research Infrastructure: an Open Symposium for HECAP, Day I-V, 6-10 July (2020)

[39] Several Authors, Latin American Workshop on Software and Computing (S\&C) Challenges in High-Energy Particle Physics (HEP), Mexico City, 20-23 November (2019)

[40] Snowmass 2021, https://snowmass21.org/start

[41] European Strategy Group, 2020 Update Of The European Strategy For Particle Physics, European Strategy Update, ISBN: 978-92-9083-575-2, 1-11, (2020)

[42] The European Strategy Group, Deliberation Document On The 2020 Update Of The European Strategy For Particle Physics, ISBN: 978-92-9083-576-9, 1-19, (2020) 


\title{
6. Electroweak \& Strong Interactions, Higgs Physics, CP \& Flavour Physics and BSM
}

\author{
M. Cambiaso (Universidad Andrés Bello, Chile) \\ E. Carrera (U. San Francisco de Quito, Ecuador) \\ A. Gago (Pontificia Universidad Catótica del Perú, Peru ) \\ M. Mulders (CERN, Switzerland) \\ R. Rosenfeld (IFT-UNESP, Brazil) \\ A. Zerwekh (U. Técnica Federico Santa María, Chile)
}

\subsection{Introduction}

These are exciting times for Particle Physics. In recent years a multitude of experimental results and advances in theoretical calculations and ideas have yielded unprecedented clarity and detailed understanding of the fundamental forces and building blocks of Nature.

In particular, the current generation of flagship collider facilities and experiments has allowed exploration at the high-energy and luminosity frontier. A vibrant participation from the scientific community in Latin America has played an important and growing role in these endeavours.

After several decades the last missing particle of the Standard Model (SM) of particle physics was finally detected in 2012: the Higgs boson. It is amazing to see the rapid development in the Higgs measurements. Figure 6.1 shows the first lines of the Higgs boson entry in the 2020 PDG. Notice the mass average of $m_{H}=125.10 \pm 0.14$ - a $0.1 \%$ measurement! The couplings of the Higgs boson are also in very good agreement with the SM. Figure 6.2 presents a recent CMS result that includes the first $3 \sigma$ measurement of the Higgs couplings to muons, the first time that there is direct evidence that the Higgs interacts with a 2nd generation particle.

Furthermore, one should also emphasize the very good agreement among precision measurements of electroweak observables with theoretical calculations, as exemplified in Figure 6.3

Despite its tremendous success in explaining a huge amount of processes studied in different HEP facilities, the SM has shortcomings that point to its incompleteness as the ultimate model of Nature. Some of the shortcomings of the SM are:

1. origin of its 19 free parameters (related to flavour and CP problems);

2. origin of neutrino masses;

3. origin of dark matter;

4. origin of dark energy;

5. hierarchy or naturalness problem;

6. does not contain gravity.

In particular, the SM describes only roughly $5 \%$ of the components of the Universe. It is known that we do not know what $95 \%$ of the universe is made of; and that is a big gap. Dark matter and dark energy, contributing with $25 \%$ and $70 \%$ to the energy density of the Universe today respectively, are not described by the SM. In addition, we know that neutrinos have masses and this is also not contained in the SM.

The hierarchy or naturalness problem deserves more explanation. The Higgs boson mass in the SM receives large contributions from radiative corrections due to some new physics appearing at a higher energy scale $\Lambda$. Therefore an exquisite fine-tuning of bare parameters in the Lagrangian is necessary to explain why $m_{H} \ll \Lambda$. This problem, although a theoretical one, is the main guide to build models beyond the SM (BSM). We can 


$$
J=0
$$

In the following $H^{0}$ refers to the signal that has been discovered in the Higgs searches. Whereas the observed signal is labeled as a spin 0 particle and is called a Higgs Boson, the detailed properties of $H^{0}$ and its role in the context of electroweak symmetry breaking need to be further clarified. These issues are addressed by the measurements listed below.

Concerning mass limits and cross section limits that have been obtained in the searches for neutral and charged Higgs bosons, see the sections "Searches for Neutral Higgs Bosons" and "Searches for Charged Higgs Bosons $\left(H^{ \pm}\right.$and $\left.H^{ \pm \pm}\right)$", respectively.

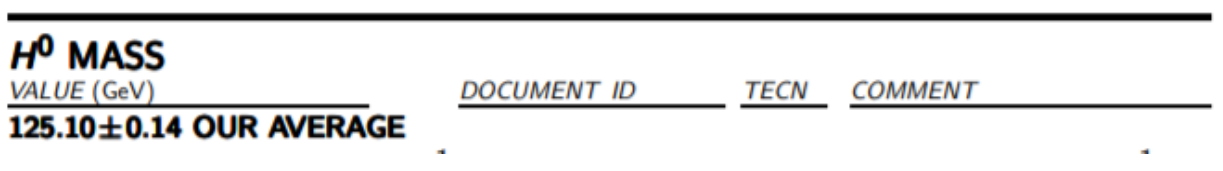

Figure 6.1: First page of the Higgs boson entry in the 2020 PDG.

classify the BSM proposals as:

- Supersymmetric (SUSY) extensions of the SM: Higgs mass protected by SUSY;

- Composite Higgs: Higgs mass protected by a shift symmetry;

- Extra dimension: Higgs mass protected by the geometry of space-time.

However, the naturalness problem as a guide to BSM has been challenged by the absence of new physics at the LHC. As shown in Figure 6.4 energy scales of the order of $10 \mathrm{TeV}$ have already been explored. We may be entering the post-naturalness era [1].

There are also challenges related to the strong interactions. The SM contains the basic ingredients for the description of hadrons in terms of their fundamental constituents, quarks and gluons. The interaction between quarks occurs through gluons, which also interact with each other. QCD has been very successful in describing a large variety of physical phenomena, from the most basic properties of nuclei found in nature to extreme conditions, such as the dynamics inside neutron stars or collisions between elementary particles, either in the atmosphere (cosmic rays) or in large particle accelerators. Two important features of QCD are asymptotic freedom and confinement. The first principles understanding of confinement is still an outstanding open question. An important way to study confinement is to create a deconfined medium in high energy heavy ion collisions. In extreme situations of temperature and/or baryon density, confinement in hadrons is no longer a necessary feature of the medium. In this sense, a phase transition to more complex states of matter occurs. The study of these states is extremely important for the complete understanding of the equation of state of QCD and its properties.

In particular, when the baryonic density is low and the temperature is high, a phase transition is expected to form a deconfined state of quarks and gluons called Quark-Gluon Plasma (QGP). The observation and characterization of this state has important consequences for the detailed understanding of the strong interactions and it is also crucial for a better comprehension of the evolution of our Universe since it is expected that it was in a similar state after a few moments from the Big Bang. The observation and investigation of this state of matter allows us to understand in more detail the evolution of the primordial Universe, its expansion and hadronization. The experimental investigation of the existence and characterization of this state of matter is the focus of large accelerators of heavy ions at relativistic energies, such as RHIC (Relativistic Heavy lon Collider) in the USA and LHC (Large Hadron Collider) in Europe.

This chapter focuses on the Latin American contribution to the advancements in the study of the electroweak and strong interactions, Higgs physics, the origin of CP violation, the origin of flavour, and new physics beyond the Standard Model. The main physics drivers are:

1. precision tests and detailed exploration of the Standard Model; 


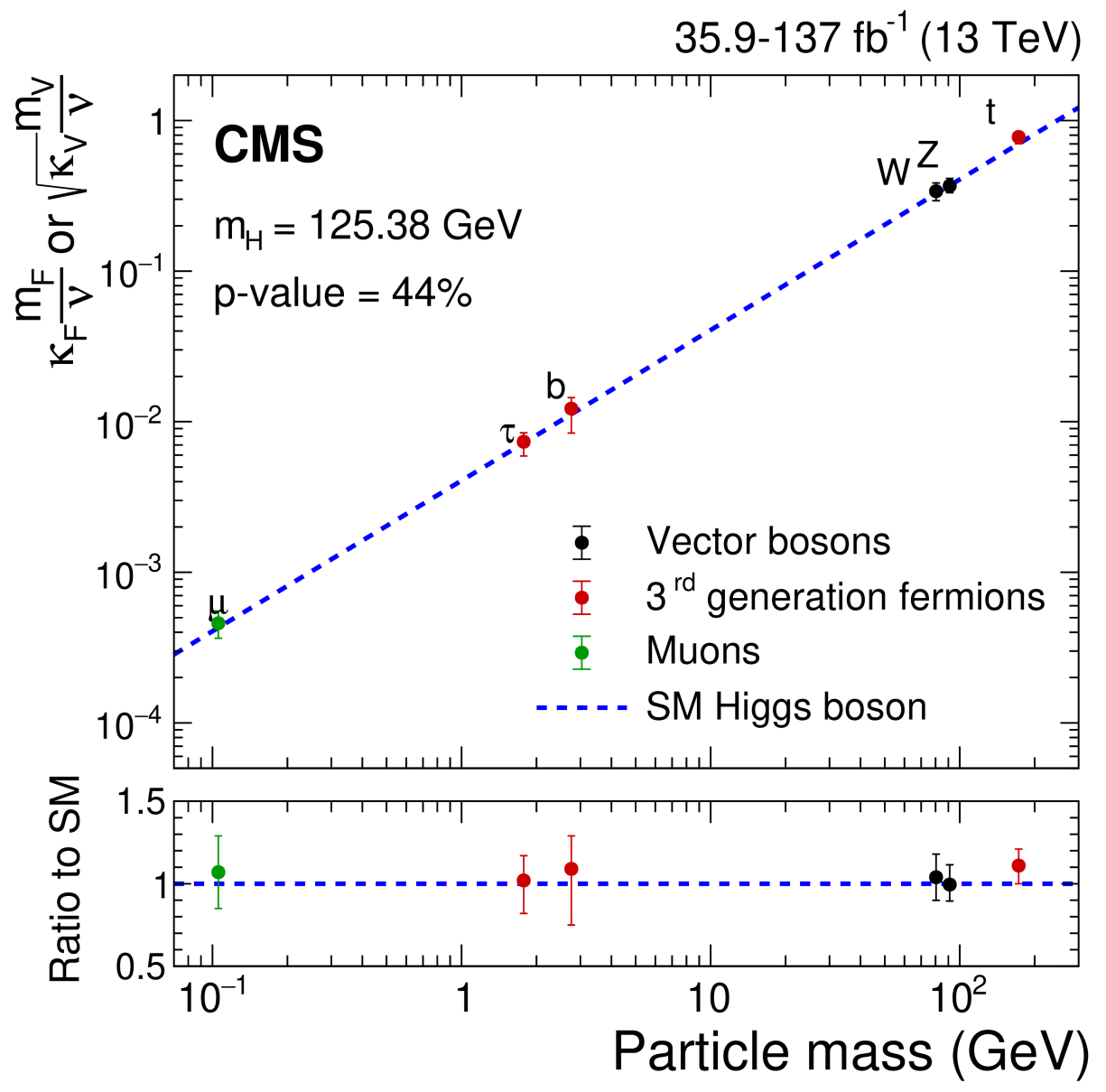

Figure 6.2: Reduced coupling-strength modifiers $\kappa_{F} m_{F} / v$ for fermions $(\mathrm{F}=\mathrm{t}, \mathrm{b}, \tau, \mu)$ and $\sqrt{\kappa_{V}} m_{V} / v$ for weak gauge bosons $(\mathrm{V}=\mathrm{W}, \mathrm{Z})$ as a function of their masses $m_{F}$ and $m_{V}$, respectively. The SM prediction for both cases is also shown (dotted line). The error bars represent $68 \% \mathrm{CL}$ intervals for the measured parameters. The coupling modifiers are measured assuming no BSM contributions to the Higgs boson decays, and the SM structure of loop processes such as ggF and $H \rightarrow \gamma \gamma$. The lower inset shows the ratios of the values to their SM predictions. From CMS-HIG-19-006.

2. study properties of the quark-gluon plasma;

3. better understanding of the proton structure through improved parton distribution functions;

4. testing the properties of the Higgs boson;

5. exploring new sources of $\mathrm{CP}$ violation that could explain the matter-antimatter asymmetry in the universe;

6. search for dark matter candidates;

7. search for signals of the existence of compact extra dimensions;

8. search for SUSY;

9. testing models for the origin of neutrino masses;

10. search for other new physics beyond the Standard Model.

In the following we will describe the Latin American participation in the different experimental facilities in the region and in the world where the physical drivers mentioned above are studied. The main participation of Latin American countries in HEP/Nuclear experiments is illustrated in Figure 6.5

The majority of the HEP community in Latin America is participating in CERN experiments. More than 500 scientists, graduate students and engineers associated to institutes in the Latin American region are officially associated with CERN, predominantly as members of the LHC collaborations but also in some cases in other, smaller size experiments. Out of those, about 150 participants are considered Authors, and appear as authors 


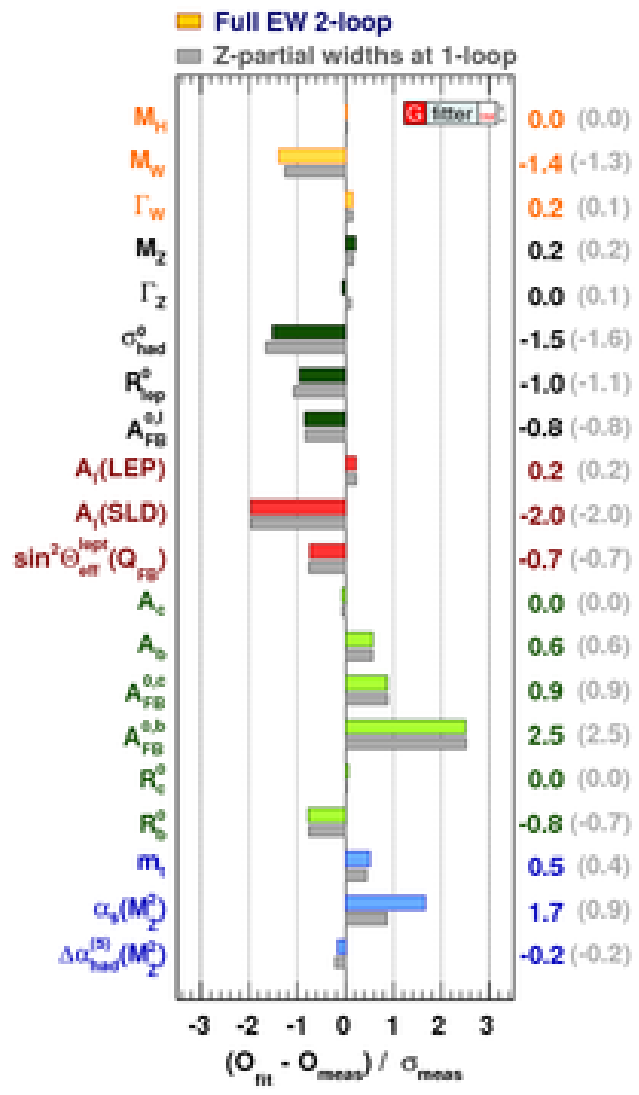

Figure 6.3: Comparing fit results with direct measurements: pull values for the SM fit, i.e. deviations between experimental measurements and theoretical calculations in units of the experimental uncertainty. [Figure from the GFitter group.

on scientific publications. The exact criteria for authorship depends on the experimental collaboration, but generally reflect a level of scientific, financial or technical contributions. In Table 6.1 the participation is listed, separated according to country and experiment.

\subsection{Participation of LA groups in HEP Activities}

\subsubsection{Nuclear Physics}

There are two particle accelerators dedicated to basic nuclear physics in South America: the one at the Open Laboratory for Nuclear Physics (LAFN) at the Institute of Physics of the University of Sao Paulo (IFUSP) in Brazil and another Pelletron tandem, called Tandar, at Commission Nacional de Energia Atomica (CNEA) in Buenos Aires, Argentina. LAFN is equipped with a Tandem Accelerator, Pelletron 8UD, which produces stable beams of energies up to $5 \mathrm{MeVA}$.

The experimental hall of LAFN has several beamlines with different equipment: large multipurpose scattering chamber, special beam line for applications, Enge Split-Pole magnetic spectrometer, HPGe array with mini-ball for particle detection. In 2004, the RIBRAS system, acronym standing for Radioactive lon Beams in Brasil, became operational at LAFN, connected to the $8 \mathrm{MV}$ Pelletron accelerator. It delivers light radioactive ion beams, such as $\mathrm{He}, \mathrm{Li}, \mathrm{Be}, \mathrm{Be}, \mathrm{B}$, produced by transfer reactions, which are separated, and focused by two superconducting solenoids. It is the first such equipment in the Southern Hemisphere and the only one in Latin America.

LAFN has about 60-70 users including staff members, post-docs, graduate students and external users. The main interest is in nuclear reactions with weakly bound projectiles, stable or radioactive. 
Table 6.1: Participation of scientists and engineers from institutes in the Latin American region in CERN experiments [Source: CERN Greybook database (as of September, 2020)]

\begin{tabular}{|c|c|c|c|}
\hline Country & Involvement & Participants & Authors \\
\hline Argentina & ATLAS & 23 & 5 \\
\hline \multirow[t]{9}{*}{ Brazil } & ALICE & 50 & 7 \\
\hline & ATLAS & 67 & 8 \\
\hline & CMS & 70 & 32 \\
\hline & $\mathrm{LHCb}$ & 39 & 19 \\
\hline & ALPHA & 5 & 3 \\
\hline & ISOLDE & 12 & 4 \\
\hline & AMS & 2 & 0 \\
\hline & ProtoDune & 6 & 0 \\
\hline & RD51 & 5 & 1 \\
\hline \multirow[t]{5}{*}{ Chile } & ATLAS & 70 & 9 \\
\hline & CLIC & 2 & 0 \\
\hline & ISOLDE & 5 & 1 \\
\hline & NA64 & 7 & 2 \\
\hline & SHiP & 6 & 0 \\
\hline \multirow[t]{5}{*}{ Colombia } & ATLAS & 7 & 2 \\
\hline & CMS & 21 & 6 \\
\hline & ISOLDE & 2 & 2 \\
\hline & LHCB & 3 & 3 \\
\hline & RD51 & 4 & 3 \\
\hline Costa Rica & ISOLDE & 1 & 1 \\
\hline Cuba & ALICE & 5 & 1 \\
\hline Ecuador & CMS & 9 & 0 \\
\hline \multirow[t]{3}{*}{ Mexico } & ALICE & 82 & 14 \\
\hline & CMS & 41 & 18 \\
\hline & NA62 & 5 & 2 \\
\hline Peru & ALICE & 12 & 3 \\
\hline \multirow[t]{6}{*}{ LA Region } & 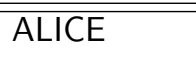 & 108 & 29 \\
\hline & ATLAS & 167 & 24 \\
\hline & CMS & 141 & 56 \\
\hline & LHCB & 42 & 22 \\
\hline & OTHER & 103 & 15 \\
\hline & TOTAL & 561 & 146 \\
\hline
\end{tabular}




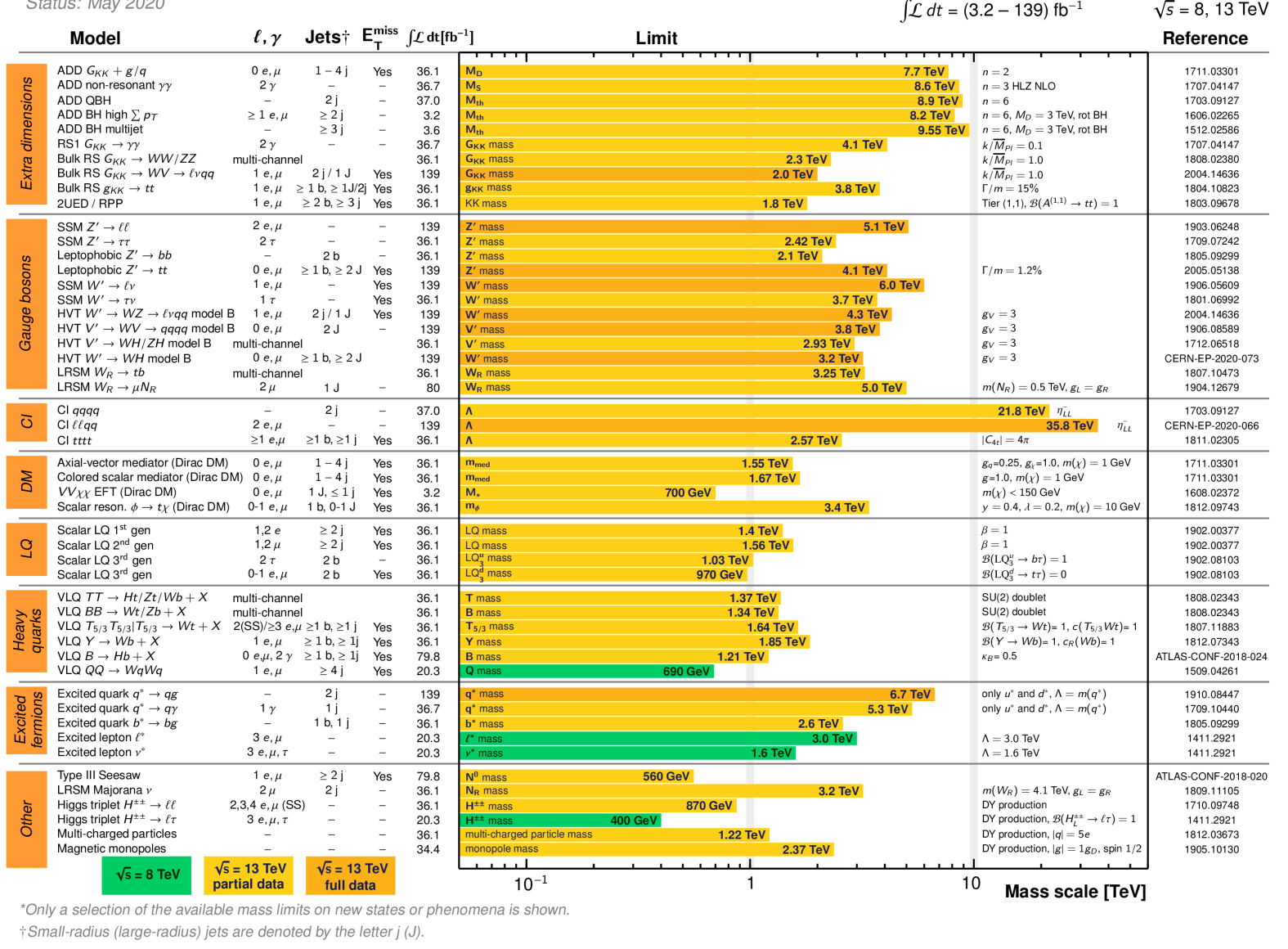

Figure 6.4: Bar chart with the search of particles predicted by several BSM proposals. Notice that energy scales of the order of $10 \mathrm{TeV}$ have already been explored at the LHC without signs of new physics. From ATLAS Exotics Group (2020).

The nuclear physics community in Brazil has 35 institutions, 140 researcher members and roughly 60 students and post-doctoral fellows working in three large areas:

- 62 researchers in High Energy Nuclear Physics (HENP)

- 31 researchers in Nuclear Structure and Reactions (NSR)

- 47 researchers in Nuclear Physics Applications (NPA)

In HENP, the activities are

Theory

- Hadron theory, effective models, QCD sum rules, among others. (17 researchers)

- Compact stars, EOS with quarks and hadrons, Strong Magnetic field, among others. (14 researchers)

- Relativistic heavy ions, hydrodynamics, Quark- Gluon Plasma, among others. (10 researchers)

- QCD phenomenology, low $x$ and Color Glass Condensate. Diffractive pp scattering, among others. (9 researchers)

- QCD theory, Confinement and Critical Point, Lattice QCD, eq. Dyson-Schwinger, among others. (6 researchers)

\section{Experiment}

- Ultrarelativistic heavy ions, hydrodynamics, QGP, etc. (3 researchers)

- 2 Researchers at UFRJ of the Auger Collaboration and CONNIE neutrino experiment

There are very few experimentalists working in Hadron Physics in Brazil, and most LHC and RHIC experimentalists are not part of nuclear physics projects. 


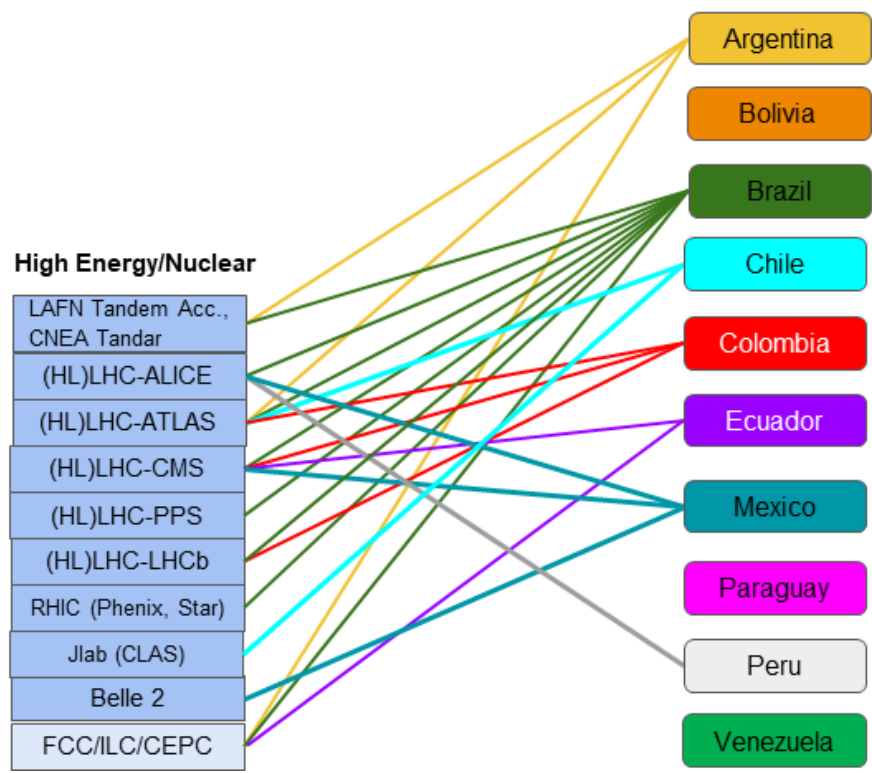

Figure 6.5: Landscape of LA participation in HEP/Nuclear experiments.

\subsubsection{Jefferson Laboratory}

A Chilean group participates in the CLAS collaboration in Jefferson Lab. It is composed of two faculty professors, four postdocs and six graduate students. Its contribution has two primary thrusts: analysis of the EG2 dataset for deep inelastic scattering on nuclei, and development of hardware projects for future CLAS experiments.

The CLAS EG2 dataset is the world's largest sample of deep inelastic scattering on nuclei with identified hadrons. The Chilean analysis activity includes studies of multiplicity ratios, transverse momentum broadening, two-pion Bose-Einstein correlations, and short-range correlations. Recently, for instance, it finalized an analysis of the eta meson multiplicity ratio in two decay modes $\left(\eta \rightarrow \gamma \gamma\right.$ and $\left.\eta \rightarrow \pi^{+} \pi^{-} \pi^{0}\right)$. Members of the group have strongly advanced the measurement of the multiplicity ratio for the omega meson. The results are clearly promising for this world's-first measurement.

There are four activities for hardware development. A dual-target has been developed for the future deep inelastic scattering experiment on nuclei with CLAS (E12-06-117) in an experiment that has four co-spokespersons who are members of the Chilean group. This system allows rapid changes of solid targets for fixed-target experiments with a closely proximate cryogenic target. This activity has included the construction and testing of the final system based. The second activity has been the installation and upgrade of the BAND (Backward Angle Neutron Detector), which will be used in the same experiment as well as in experiment E12-11-003A for two-nucleon correlations studies that include detection of backward-angle neutrons. BAND has been collecting data during much of the first operations of CLAS12. The third activity has been the development of lightweight mirrors for future Ring- Imaging Cherenkov detectors for CLAS, which will benefit the above experiments as well as many others, such as E12-09-007. This effort has been strengthened by close collaboration with a group developing mirror technology for astronomy. The fourth activity is to develop polarized target technologies to be used in experiment E12-14-001.

\subsubsection{LHC-ATLAS}

Nine institutes from Latin America, approximately 50 scientists and engineers from Argentina, Brazil, Chile and Colombia, had worked on ATLAS since well before data-taking started, making important technological and software contributions to the instrument. Latin American universities have so far awarded close to $90 \mathrm{PhD}$ and MSc-level degrees for work on ATLAS, and work is in progress for many more. Latin American researchers have made significant, and in many cases highly visible, contributions to ATLAS across the spectrum of activities: 
detector and trigger operations, the data processing chain, software development, reconstruction of important particle signatures, physics analysis, and detector upgrade developments.

In terms of detector development activities, the ATLAS hadronic calorimeter system has significant contributions from Brazilian institutes. Chile is one of five countries producing muon detector chambers using novel technologies for the upgrade of the ATLAS muon system. Institutes from Argentina and Colombia have made important contributions to the ATLAS trigger and data acquisition system.

In Argentina, HEP groups have been members of ATLAS since 2006. Argentina is committed to the ATLAS upgrade for the HL-LHC with contributions to the trigger electronics, Data Acquisition, High-Level trigger software and hardware, and photon/electron/tau/jet reconstruction. The responsibilities in ATLAS have included so far participating in the online testing of data taking, the development of the programs for the online selection of events, the calibration of detectors, the measurement and improvement of their performance, as well as the pursuit of multiple lines of physics analysis, several of them as leaders of the respective analysis teams.

The main interests have concentrated on event triggering, reconstruction and physics analyses based on photons and/or jets. These have included SM precision measurements, searches for supersymmetry and other BSM models. Several PhD thesis in the group have addressed these analyses.

The Argentinian institutions are involved in the design, construction and tests of the Global Trigger component of the first part of the trigger, the hardware-based Level- 0 with the commitments:

- Design, production and testing of Common Modules (a common hardware module with different functionality implemented in firmware, composed of ATCA blades and FPGAs with many multi-gigabit transceivers);

- Development of algorithmic firmware and software;

- Design, production, testing; installation and commissioning of Rear Transition Modules (RTM);

- Design, production, testing, installation and commissioning of Fibre Management;

- Procurement of general infrastructure.

A new HEP laboratory is being set up at Instituto de Fisica La Plata (IFLP, UNLP-CONICET). The first stage consists mainly of an electronics laboratory to develop and deploy hardware for high speed, real-time signal processing. The laboratory is being equipped and is expected to be fully operational by the end of 2020 . Some of the equipment that this lab will have when fully operational includes:

- FPGA evaluation kits

- High-performance computers for firmware synthesis

- Soldering station and tools for handling fine pitch PCBs SMD components

- High-speed oscilloscopes and high-speed function generators

- ATCA shelf

- High-speed optic fibers management.

The Argentine ATLAS groups are also involved in applying Machine Learning techniques in current and nearfuture analyses (for instance, in jet tagging, fast simulation, calorimeter calibration, and more), and foresee a much heavier involvement and usage in Artificial Intelligence tools. This requires preparation, both in terms of peripheral computing and software power but also in terms of education and supporting human resources. However, one difficulty mentioned in the white paper is the current lack of computer resources.

The ATLAS/Brazil Cluster is composed of physicists, engineers and computer technicians from UERJ, UFBA, UFJF, UFRJ, and USP and started research activities at CERN in 1988. The Cluster is involved in both electronic instrumentation and algorithm development for calorimetry. The activities aim at developing the new back-end electronics (FPGA-based) and new energy estimation algorithms based on deconvolution strategies to be used both online and offline. For the electromagnetic calorimeter (Lar), activities are mainly related to the implementation of the Super Cells, which refer to a new trigger piece of hardware. Additionally, signal processing and machine learning techniques are being applied for cross-talk mitigation.

Additional (level one) trigger from the Tilecal last segmentation layer is currently being provided for the extended barrel part of ATLAS. It may also cover the barrel so that the muon trigger may be assisted from Tilecal information. Still, within Tilecal, an effort is being made to provide finer granularity through a multianode PMT readout. The aim is to achieve two or four times finer detector cell granularity without changing the mechanical structure of the calorimeter. Artificial intelligence and blind source separation techniques are being used envisioning operation in the second upgrade phase. For the triggering system, they participate in developing new computational intelligence-based techniques for the high-level calorimeter trigger (HLT) system, which are also to be extended to the offline filtering. Besides, efforts are being made to improve the trigger calibration for electrons. Possible extensions of the machine learning techniques developed for HLT 
to level-one triggering (embedded electronics) are also being pursued (FPGA-based designs). Concerning the physics analysis, activities include the search for ALP (Axion Like Particle) and Standard Model analysis.

The ATLAS management has mainly been structured upon the Glance/FENCE technology, which was developed by the ATLAS/Brazil Cluster. It is worth mentioning that the Brazilian industry has been participating in the construction of ATLAS, with two electronic circuit boards designed by the Cluster and produced in Brazil, which were both operating in the ATLAS trigger system until the end of the Run 2 data acquisition in 2018. Finally, the Atlas/Brazil cluster has a strong outreach component.

In Chile, there is a group working in three primary analysis. The first analysis is of the production and suppression of $J / \psi$ mesons and $\psi^{\prime}$ mesons in collisions between lead ions, with the normalization taken from pp collisions at the equivalent energy. The second is based on the study of the flow coefficient $v 2$ for these mesons. Both of these groundbreaking studies are of high international interest in the worldwide heavy-ion community. Finally, the third analysis is the search for double Higgs production in the ATLAS data.

A large, ongoing technical project with ATLAS continues to be the Chilean contribution to the muon system upgrade. This work involves producing more than 140 small-strip Thin Gap Chambers (sTGC) that will be used for triggering and precise measurement of muons. This project, a collaboration between Chile, Israel, China and Canada (with contributions and coordination from Russia and CERN) consists of fabrication and testing activities.

In Colombia, the experimental High Energy group of Universidad Antonio Nariño has been part of the ATLAS Collaboration since 2007. In these years, the group has been part of different activities, including detector performance studies and its calibration, physics analysis as well as activities related to the ATLAS detector maintenance and operation. The group is interested in searches for BSM physics such as charged Higgs, invisible Higgs, SUSY signatures, heavy neutral leptons and other possible long-lived particles. ATLAS work has focused on the e/gamma trigger, assessment and design of ATLAS Control network and upgrade of TDAQ with network engineers. Also, for the upgrade, there has been work done on the temperature sensors calibration and readout for the ITk. A new detector laboratory has been built at this institute over the last few years focused on R\&D for SiPMs and MPGDs.

\subsubsection{LHC-CMS}

In total, 12 groups from Latin America (Colombia, Ecuador, Brazil, Mexico) contribute to CMS with almost 50 scientists, representing roughly 3.5\% of the collaboration. Latin American countries have so far awarded around $60+\mathrm{PhDs}$ and MSC-level degrees for working in CMS, and work is in progress for many more. The collaboration with CMS was instrumental in establishing the Master- and the PhD programme in some institutes. There are also many engineering and computing students/specialists that are working in CMS.

Latin American researchers have made significant, and in many cases highly visible, contributions to CMS across the spectrum of activities: Detector hardware, data taking at CERN, operation, maintenance and upgrade, physics analysis, data processing including validation and certification, computing with the operation of Tier-1, Tier-2 and Tier-3 sites, and central software developments.

The Latin American countries and institutes contribute crucially to various detector systems in CMS, for example, in the area of the Muon detectors in two different technologies and their upgrades in hardware and especially in electronics. They also contribute to the HCAL (hadronic calorimeter), the beam radiation monitoring system BRIL, as well as to the very forward Precision Proton Spectrometer (PPS).

In Brazil, there is a group of roughly 30 researchers from CBPF, UERJ, UFABC and UNESP working in CMS. A Tier 2 of the WorldWide LHC Computing Grid (WLCG)is hosted at UNESP. The group has had a key role in the CMS-TOTEM Precision Proton Spectrometer (CT-PPS). They have also participated in the analysis of monojet events, heavy-ion physics and vector-boson fusion.

In Colombia, the High Energy Physics group of Universidad de Los Andes (UNIANDES), has been a full member of the CMS collaboration since March 2006, contributing both in the detector operation and physics analysis. The group has been involved in the operation and upgrades of the resistive plate chambers (RPC) and gas electron multiplier detectors (GEMD) used for muon identification and triggering. About 30 engineers from UNIANDES have been part of the international team in charge of the computing and data operations, through the GRID system, of the CMS experiment. Some of the engineers have been stationed for about two years at CERN, others at FERMILAB. The group has also been heavily involved and leading searches of supersymmetric signatures and physics beyond the Standard Model. Few of them focused on the experimentally challenging 
compressed mass spectra scenarios, which require special physics tools such as jets from initial-state radiation or vector-boson fusion topology. The UNIANDES HEP group has graduated seven $\mathrm{PhD}$ and more than a dozen MSc students. As a bridge for technology transfer to Colombia, the group has setup an instrumentation laboratory in the Bogota university campus, where interdisciplinary applications of semiconductor and gaseous radiation detectors are being investigated.

As an example from the white papers, in Colombia, the group of Phenomenology of Fundamental Interactions of Universidad de Antioquia (UDEA) has been working for several years in phenomenology. Since 2019 the group is a member of the CMS Collaboration. They have been working on searches for physics beyond the Standard Model. Currently, three professors, one post-doc, two Phd students, one master student and two undergraduate students, and members of the group are associated to CMS projects.

In Ecuador there are groups at Escuela Politécnica Nacional (EPN) in Quito and Universidad San Francisco de Quito (USFQ) participating in CMS. Ecuadorian scientists and engineers have contributed to the High Luminosity Trigger, the Beam Radiation Instrumentation and Monitoring (BRIL) subsystem and are also deeply involved in the data preservation and open access activities. Several analysis addressing top physics, Higgs physics and exotic particles searches have had Ecuadorian contributions. The community has of the order of $30 \mathrm{PhDs}$ in HECAP and expressed interest in joining one future collider experiment in the energy frontier, such as the ILC, FCC.

In Mexico there is a group of $15 \mathrm{PhDs}$ contributing with $\mathrm{M} \& \mathrm{O}$ to the CMS experiment. CMS-Mexico is heavily involved in the Resistive Plate Chamber System part of the CMS Muon system and in the Beam Radiation, Instrumentation, and Luminosity (BRIL) system since (2019). Universidad Iberoamericana is one of the Institutes responsible for the improved Resistive Plate Chamber (iRPCs) Production for the Phase II Upgrade. Benemérita Universidad Autónoma de Puebla (BUAP) is responsible for the Detector Control System of the current Resistive Plate Chambers system and the corresponding for the validation of the iRPCs. BUAP, CINVESTAV and Universidad Iberoamericana participated in the build and test of the first prototypes of the iRPCs. At Universidad Iberoamericana in Mexico city a laboratory for R\&D and for the construction and validation for iRPC's will be completed by the end of 2020. CINVESTAV holds a TIER-3 cluster for CMS-GRID, while BUAP is in the process of certification to hold a TIER-3 cluster too. Universidad Iberoamericana is responsible to coordinate the Simulations \& Performance Estimations subgroup of the BRIL Phase-2 Upgrade Group. Universidad de Sonora is involved in the BRIL project taking leading roles in tasks related to the estimation of the radiation environment, luminosity measurements in $\mathrm{pp}$ and $\mathrm{Pb}-\mathrm{Pb}$ collisions, and coordinating the Online Luminometry group for Phase-2 upgrade. Physics analyses where Mexicans are leading and participating include Heavy Flavor Physics, Quarkonia, Higgs, Top, Heavy lons and Beyond the Standard Model Physics.

\subsubsection{LHC-LHCb}

In Brazil, researchers from CBPF, UFRJ and PUC-Rio are members of the LHCb collaboration since 1998. The group today has around 20 faculty. During its 20 -years long participation in $\mathrm{LHCb}$, the Brazilian team gave several crucial contributions, with key roles in the development, installation and commissioning of the Muon System, notably in the readout electronics, multi-wire proportional chamber design and characterization, and experiment control system. The Brazilian groups developed the first muon identification algorithms that played a major role in LHCb flagship measurements. Brazil gave important contributions also in trigger (L0 and HLT), and computing. Brazilian physicists are contributing substantially to several physics analysis projects. There was a recent review on direct $\mathrm{CP}$ violation in beauty and charm hadron decays authored by 2 Brazilian members of the collaboration [2].

The Brazilian team is involved in the development of two new detectors for the LHCb upgrade which will be installed in the Long Shutdown 2 of LHC in 2019-2021:

- the upgrade of the VELO. This new detector features silicon pixel sensors and a state-of-the-art readout chip with very high data throughput. Sensors and readout ASICS are placed very close to the LHC beam ( $5 \mathrm{~mm}$ ) and operated in vacuum, in a very high radiation environment. The group contributes to the test of the pixel sensors, to the design, optimization and tests of the detector system and to the highly sophisticated motion system;

- Scintillating Fibre Tracker (SciFi). This is the largest scintillating fibre tracker ever built and operated. Brazil is contributing to the development and test of the so-called front-end boards, hosting the ASICS that read out the Silicon Photomultiplier arrays connected to the scintillating fibres.

Brazil is providing valuable computing resources through a TIER2 for the GRID. The main challenges mentioned 
are the increasing costs for $\mathrm{M} \& \mathrm{O}$ and mobility.

In Colombia, the Universidad Nacional de Colombia (UNAL) was accepted to participate as an associated group of LPNHE (Laboratoire de physique nucléaire et de haute énergies) in the LHCb Collaboration in 2014. The group is composed of two professors, three undergraduate students, four master students and one PhD student. Their members are collaborating in the physics of charm quarks, quarkonia and exotic states analyses.

\subsubsection{LHC-ALICE}

Scientists from Latin America (from 13 institutions from Brazil, Chile, Colombia, Mexico and Peru) make up 5\% of the collaboration. A major contribution to the ALICE upgrade is the design and production of the SAMPA chip, a dedicated analog/digital ASIC for the readout of the $500 \mathrm{k}$ channels of the TPC and $1 \mathrm{M}$ channels of the tracking chambers of the Forward Muon Spectrometer, that was developed and funded in Brazil. Another of the key contributions to the upgrades is a scintillator detector with a high time resolution called $\mathrm{V} 0+$, which is part of the Fast Integration Trigger and is developed and funded in Mexico.

In Brazil, there is a group of approximately ten physicists from the University of São Paulo (USP), the University of Campinas (UNICAMP), the Federal University of ABC (UFABC), and the Federal University of Rio Grande do Sul (UFRGS) working in ALICE.

The ALICE groups in Brazil have been providing their fair share contribution for computation to the experiment since 2007 through the SAMPA cluster (acronym from the Portuguese, Sistema de Análise e MultiProcessamento Avançado), hosted at USP. Currently, the cluster has 2408 CPU cores, totalizing 18.7 kHS06 of processing power and $0.85 \mathrm{~PB}$ of storage. However, it is important to highlight that part of this processing capability, around $6 \mathrm{kHS06}$, is used by local users, outside the GRID. And an increase in computing power and data storage will be needed for Run-3.

For the Run-3 upgrade, the Brazilian groups have provided major contribution through the full development of a new front-end readout SAMPA chip for two ALICE detectors, the Time Projection Chamber (TPC) and the Muon Chamber $(\mathrm{MCH})$, and the construction of the mechanical support of the new Muon Forward Tracker (MFT).

Recently, the ALICE collaboration proposed an upgrade for the LHC Run-4 to build a Forward Calorimeter (FoCal). The Brazilian group plans to contribute to the R\&D and construction of the FoCal Silicon Pad layers readout system. Given the large experience of the group with the development of the SAMPA chip, which will instrument the ALICE TPC and MCH detectors beyond Run-3, it is natural to expect that a significant contribution to this part of the project can be achieved. They also plan the study of aging due to the surface chemical reaction that occurs at the electrodes inside the Gas Electron Multiplier (GEM).

In Mexico, there is a group of $15 \mathrm{PhDs}$ contributing with M\&O to the ALICE experiment. CINVESTAV and Universidad Autónoma de Sinaloa are responsible for the design, construction and operation of the Forward Diffractive Detector (FFD). This detector is now in the construction phase. ICN-UNAM is responsible for the design, construction and operation of a pico-amperemeter for the TPC, in collaboration with BUAP. This employs new technology, and there is already a prototype tested in Mexico and CERN, with the final instrument expected for the end of 2020. In addition, it holds a TIER-2 cluster for ALICE-GRID. IF-UNAM is responsible for the V0-plus detector. Ten prototypes have been built and tested at IF-UNAM and CERN. The construction of all parts of the detector, which contains 50k optical fibers, was finalized in October 2019 and shipped to CERN for final assembly. BUAP has also been responsible for the operation of the ACORDE detector and the design and construction of the CTP (Central Trigger Processor) in collaboration with the University of Birmingham. The physics analyses where Mexicans have active visibility are in cosmic rays, diffractive physics, multiplicity measurements and underlying events.

In Peru the PUCP (Pontificia Universidad Católica del Perú) started working at ALICE in 2004. Since then, the group has contributed to the development of an analysis tool for the software, detector design, performance studies, and many more. In particular, the group has had a major role in the design, performance studies, and the test-beam experiments of the ALICE diffractive (AD) detector system. The AD detector increases the ALICE detector's forward physics coverage and its precision on the measurements of the inclusive diffractive cross-sections. Currently, they have a leading role in the analysis aimed to report the ALICE's updated (taking into account the $A D$ detector system) measurements of the diffractive cross-sections.

They have had contributed to the characterization tests of the CMOS Monolithic Active Pixel Sensors that are the cornerstone of the Muon-Forward-Tracker (MFT), a Si-tracking detector part of the ALICE upgrade 
programme planned to be working after the Second Long Shutdown. More recently, and still in the context of the MFT, the PUCP team is contributing in the implementation of the geometry of the MFT within the new ALICE computing model.

\subsubsection{SuperKEKB}

Mexico requested to join the Belle II experiment in SuperKEKB in 2013. There are currently 8 faculty researchers and $6 \mathrm{PhD}$ students from three institutions: CINVESTAV IPN, UAS and UNAM (IF). A tier 2 center for Belle II has been online since May 2015. The group is developing electronics for the Large-angle beamstrahlung monitor (LABM). The group participated in the $\tau \rightarrow 3 \pi v$ analysis and will concentrate on the search for Lepton Flavor Violation in $\tau$ decays.

\subsubsection{Future Colliders}

Despite the LHC success, there are still many open scientific questions regarding the fine details of the electroweak and strong sectors of the SM and its possible projections into a new, more complete framework for the understanding of the Universe. Therefore, the majority of groups have special interest in continuing their efforts during the period of operation of the upgraded, high-luminosity version of the accelerator, the HL-LHC [3]. The LHC has a clear schedule until 2035. There is a clear understanding, among Latin American researchers, that the medium and long-term future of particle physics lies mostly on the next generation of colliders, whose construction is currently being discussed at the international, global scale.

While the HL-LHC is already approved [4], the next generation of colliders are still under scrutiny. The reference point for the development of such large scale devices will be, precisely, the HL-LHC. Latin American groups have expressed their interest in the high-energy version of the LHC (HE-LHC) [5], all the possible versions of the Future Circular Collider [6] (i.e., ee, eh and hh), the Circular Electron-Positron Collider (CEPC) [7], the International Linear Collider [8], the Compact Linear Collider (CLIC) [9], and the Large Hadron electron Collider [10] ( $\mathrm{LHeC}$ or $\mathrm{HE}-\mathrm{LHeC})$. A recent reference [11] presents a summary of the timeline and parameters of these future accelerators (see Figure 6.6)

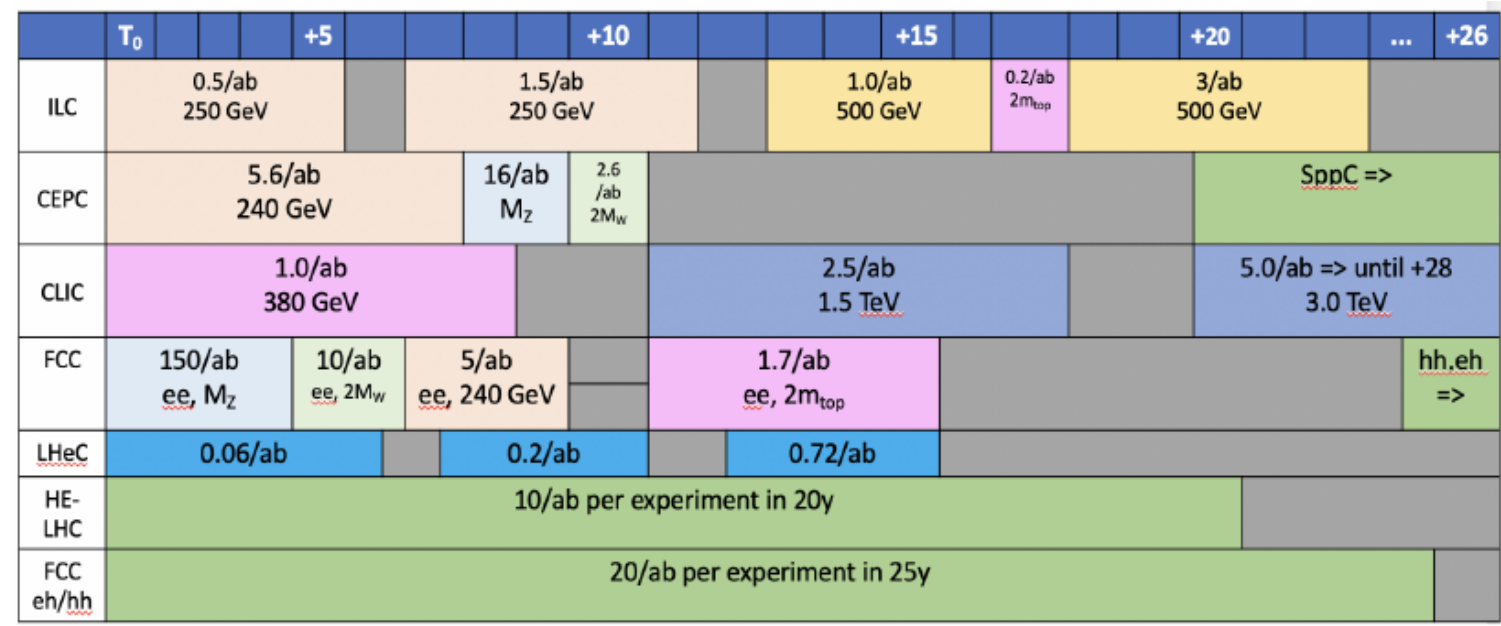

Figure 6.6: Timeline (starting at a future time $T_{0}$ ) of various collider projects. For details, see reference [1].

A white paper presented by a group of 16 young experimentalists working in Brazil details their interest in the participation in the next generation of collider experiments. In particular, they aim at organizing the community of experimental HEP in Brazil to become a member of only one large experimental collaboration in the next high-energy collider, joining forces to contribute to the development, construction, commissioning and operations of the detector as a coordinated effort. In particular they would like to avoid very specific short-aim and small-visibility projects and favour longer-term contributions that can make a greater impact within the chosen collaboration and in our research field. They estimate the computing requirements for each possibility of future experiments and foresee difficulties with the funding of such endeavours that should start to be addressed. 
Argentina ATLAS group has shown interest in $\mathrm{FCC}_{h h}$, and Ecuador also considers joining one next-generation experiment. There is clearly room for a joint LA effort in this direction.

Here we highlight the opportunities that these large scale experiments would bring about in the context of the current SM and BSM physics interest in the region and their extensions into the future.

Many groups in Latin America are deeply involved in the pursuit of a deeper understanding in Higgs physics. Among the Higgs properties that generate greater interest in the region are the couplings to other SM particles (and posibly BSM ones) and to itself. The latter characteristic plays a key role in determining the Higgs potential experimentally, which is of high priority for all future colliders. In the framework of the Effective Field Theory, the most direct way to explore the Higgs cubic self-interaction is through the study of double Higgs production, which has notable interest within the Latin American community. While these studies will advance greatly during the HL-LHC rule, it will take the next generation of colliders to establish unequivocally the existence of such self-interactions and, even more remarkable, to start probing the realm of quantum corrections to the Higgs potential [11].

Reaching evidence level for two-photon Higgs production in ultraperipheral proton and nuclear collisions could be a possibility at the future HE-LHC. Observation level of this production mechanism could be reached at the FCC. This would open and independent area of study (with leading roles in the region) for measurements of the $H-\gamma$ coupling, not based on decays but in production modes [12],

All future colliders will enhance electroweak precision measurements 13 14. Discrepancies between the electroweak precision observables and its measurements could indicate the presence of new physics. This modifications can be gauged by the so-called oblique parameters [15], which is one of the possible topics that can spark interest in the region with great potential for success.

Opportunities for dedicated flavour physics studies will be dramatically enhanced in the HL-LHC. With the expected LHCb upgrades, for instance, it will be possible to test, with unprecedented precision, CP-violating phases, transitions like $b \rightarrow s \ell^{+} \ell^{-}$and $b \rightarrow d \ell^{+} \ell^{-}$(in both muon and electron decay) and flavor violation. The rather new area of charm sector studies will also benefit from the high luminosity version of the LHC and may lead to significant discoveries [16]. In the future, with the enhanced statistics and cleaner environments at the next generation of colliders, there will be great potential to study flavor physics.

In the same context of flavor physics, groups in Latin America, which are involved in the Belle II experiment at SuperKEKB, will most likely continue their participation until completion of its research program, whose highlight is the search for lepton flavour violation. The target integrated luminosity $\left(50 a b^{-1}\right)$ for Belle II will be accumulated by 2029 [17].

The study of QCD in very dense and high temperature conditions will also benefit from this new generation of colliders [18]. The increase in luminosity and energy will allow for stronger collective signatures in QGP, the characterization of dense QCD matter through hard-scattering processes (even Higgs boson production), and the exploration of saturated parton densities in never-seen-before conditions.

FCC will give ample opportunities to exploit the regional expertise with jets in order to study parton radiation and fragmentation, jet properties and substructure, heavy quark jets and non-perturbative QCD phenomena [19].

From supersymmetry to dark matter to the search for additional dimensions, the search for physics beyond the standard model has generated great interest in the region. The increase in energy and luminosity for the next generation of colliders will enable potential discoveries that have been so far denied to us by Nature. A recent reference [20] presents a thorough study of the wide range of possibilities.

Lastly, revolutionary technologies, like those used in potential muon-muon colliders to alleviate the problem of energy loss by radiation 21 22] while keeping the clean conditions of lepton colliders, or the plasma-wakefield methods for particle acceleration [23] could take leading roles in the future of the field.

\subsubsection{Theory}

We identify a large phenomenology community in Latin America doing excellent work in precision calculations in the SM (for example QCD) and in BSM signatures (especially SUSY, Composite Higgs, Extra Dimensions, Effective Field Theories and 3-3-1 extensions of the SM). 


\subsection{Training, outreach, exchange programmes}

The membership of international experimental collaborations gives access to global networks of universities, laboratories, and eminent scientists and engineers. The structures inherent in these scientific collaborations provide natural opportunities to strengthen regional networks connecting research teams in LA countries, as well as worldwide contacts, mobility and visibility.

Exchange programs for students, scientists and engineers between LA countries and Europe in the past have had very beneficial effects for capacity building. Also, direct access to the experiments where Scientific discoveries are made, and connections with world-class scientists offer a great resource for training and capacity building of higher education in the LA region. Here we list a few examples of educational programs directly linked to the involvement in the HEP community:

- realizations of several MasterClasses in different countries to familiarize high school students with particle physics in the context of CERN's International Particle Physics Outreach Group (IPPOG);

- CERN Latin-American Schools in High Energy Physics (CLASHEP) have been occurring regularly every two years in different countries in the region, since 2001;

- several activities in HEP held at the ICTP South American Institute for Fundamental Research;

- contribution to capacity building through education programs such as LA-CoNGA.

\subsection{Areas of excellence and leadership}

We identified a few examples of excellence and leadership in the region. An incomplete list is:

- Diffractive physics where UERJ CMS group in Brazil plays a leading role in CT-PPS detector and physics;

- Analysis of three-body decays of hadrons and CP violation in LHCb;

- Precision calculation of Higgs boson cross-section

- Software development for triggers

- HPC with several Tier 2 grids in the region

In addition, the participation of LA groups in these cutting-edge experiments led to the development of important capabilities in the region. Among these capabilities, we highlight a few:

- design of a chip called SAMPA in Brazil for readout of ALICE detectors to be installed for run 3;

- design and test of Global Trigger and Rear Transition Modules for ATLAS in Argentina;

- Electronics for the Large Angle Beamsstrahlung Monitor for Belle II developed in Mexico;

- testing of Low Gain Avalanche Detectors for ATLAS in Brazil;

- expertise and leading roles in several experimental analysis in all collaborations;

- several computer clusters dedicated to these experiments are installed in the region;

- expertise in theoretical calculations in most aspects of SM and BSM processes.

\subsection{Synergies}

The SM provides a framework that has close connections to understanding the Universe at large scales, as studied in astronomy and cosmology. The search for Dark Matter is an example of such a synergy. Neutrino physics is deeply intertwined with the electroweak force, flavour physics and arguably may provide a window on BSM processes and a possible origin for the CP violation necessary to explain baryogenesis.

On the experimental side, many developments in the region, such as electronic readout capabilities, the construction of RPCs and other detector components, and software development for triggering, are common to many experiments. In addition, several analysis topics have overlap among the different groups in different experiments. An example that stands out is diffractive physics, with strong groups in CMS-Brazil and ALICEMexico. Certainly, greater coordination among the different groups can be achieved. 


\subsection{Conclusions}

Latin America has a large community of approximately 500 physicists working in High Energy Physics. Most of the community is involved in the LHC experiments, and several impactful contributions to detector design and construction have been performed. Participation in the LHC experiments provides opportunities such as:

- Sophisticated radiation-hard and high-speed electronics development;

- Firmware development for state-of-the-art FPGAs with advanced tools;

- Detector development including high-granularity silicon detectors, and ultra-fast precision timing detector technologies;

- High data volume, and high data rate distributed computing infrastructures;

- Advanced computational techniques, such as machine learning;

- And, very importantly, training of young scientists and engineers in the high- technology sector.

In 10 years of LHC operation we have had a rich harvest of physics results, documented in over 3000 LHC publications [24]. So far only $5 \%$ has been collected of the total data expected by the end of the HL-LHC for ATLAS and CMS. Additionally, ambitious upgrades of ATLAS and CMS will significantly improve the detectors beyond their current performance, and even more exciting prospects exist for LHCb and ALICE. A rich physics program until 2035 is therefore guaranteed, and the ongoing detector upgrade projects offer outstanding opportunities for the LHC groups in LA countries to participate in a more coordinated manner in beyond-the-state-of-the-art detector and electronics R\&D and production, and for new teams to get involved. In addition, it is the right time to start thinking strategically about participation in the next generation of colliders.

Latin America has a leading role in some aspects of HEP and synergies with other activities in HECAP were identified. Moreover, HEP has provided important capacity building in the region through training and outreach programs. 


\section{Bibliography}

[1] Gian Francesco Giudice. The Dawn of the Post-Naturalness Era, pages 267-292. 2019.

[2] Ignacio Bediaga and Carla Göbel. Direct CP violation in beauty and charm hadron decays. Prog. Part. Nucl. Phys., 114:103808, 2020.

[3] High-Luminosity Large Hadron Collider (HL-LHC): Technical Design Report V. 0.1. 4/2017, 112017.

[4] HI-lhc cern council approval. https://council.web.cern.ch/en/content/181

[5] Andrea Dainese, Michelangelo Mangano, Andreas B. Meyer, Aleandro Nisati, Gavin Salam, and Mika Anton Vesterinen, editors. Report on the Physics at the HL-LHC, and Perspectives for the HE-LHC, volume 7/2019 of CERN Yellow Reports: Monographs. CERN, Geneva, Switzerland, 2019.

[6] A. Abada et al. FCC Physics Opportunities: Future Circular Collider Conceptual Design Report Volume 1. Eur. Phys. J. C, 79(6):474, 2019.

[7] Mingyi Dong et al. CEPC Conceptual Design Report: Volume 2 - Physics \& Detector. 112018.

[8] Keisuke Fujii et al. Tests of the Standard Model at the International Linear Collider. 82019.

[9] T.K. Charles et al. The Compact Linear Collider (CLIC) - 2018 Summary Report. 2/2018, 122018.

[10] J.L. Abelleira Fernandez et al. A Large Hadron Electron Collider at CERN: Report on the Physics and Design Concepts for Machine and Detector. J. Phys. G, 39:075001, 2012.

[11] J. de Blas et al. Higgs Boson Studies at Future Particle Colliders. JHEP, 01:139, 2020.

[12] David d'Enterria, Daniel E. Martins, and Patricia Rebello Teles. Higgs boson production in photon-photon interactions with proton, light-ion, and heavy-ion beams at current and future colliders. Phys. Rev. D, 101(3):033009, 2020.

[13] M.L. Mangano et al. Physics at a $100 \mathrm{TeV}$ pp Collider: Standard Model Processes. CERN Yellow Rep., (3):1-254, 2017.

[14] R. Contino et al. Physics at a $100 \mathrm{TeV}$ pp collider: Higgs and EW symmetry breaking studies. CERN Yellow Rep., (3):255-440, 2017.

[15] Michael E. Peskin and Tatsu Takeuchi. Estimation of oblique electroweak corrections. Phys. Rev. D, 46:381-409, 1992.

[16] Roel Aaij et al. Physics case for an LHCb Upgrade II - Opportunities in flavour physics, and beyond, in the HL-LHC era. 82018.

[17] S. Uno. KEK and its plans. JINST, 15(07):C07016, 2020.

[18] Z. Citron et al. Report from Working Group 5: Future physics opportunities for high-density QCD at the LHC with heavy-ion and proton beams, volume 7, pages 1159-1410. 122019.

[19] Proceedings, Parton Radiation and Fragmentation from LHC to FCC-ee: CERN, Geneva, Switzerland, November 22-23, 2016, 2017.

[20] T. Golling et al. Physics at a $100 \mathrm{TeV}$ pp collider: beyond the Standard Model phenomena. CERN Yellow Rep., (3):441-634, 2017.

[21] Yuri Alexahin et al. The Case for a Muon Collider Higgs Factory. In Community Summer Study 2013: Snowmass on the Mississippi, 72013.

[22] Jean Pierre Delahaye, Marcella Diemoz, Ken Long, Bruno Mansoulié, Nadia Pastrone, Lenny Rivkin, Daniel Schulte, Alexander Skrinsky, and Andrea Wulzer. Muon Colliders. 12019.

[23] Vladimir Shiltsev and Frank Zimmermann. Modern and Future Colliders. 112019.

[24] Michelangelo Mangano. LHC at 10: the physics legacy. 2020. 


\title{
7. Instrumentation and Computing
}

\author{
Hiroaki Aihara (U. of Tokyo, Japan) \\ Reina Camacho Toro (LPNHE/CNRS, France) \\ Arturo Sánchez Pineda (ICTP/INFN/ U. of Udine, Italy) \\ Hernan Wahlberg (U de la Plata, Argentina)
}

\subsection{Introduction}

The scientific goals outlined in the previous chapters pose unprecedented experimental challenges. The unique nature and magnitude of these challenges drives technological innovation and development of new instrumentation and efficient high-performance computing solutions to meet unprecedented demands. Participation in global international collaborative efforts to develop such new technologies and exposure to state-of-the-art computing infrastructure and expertise offers great opportunities, as well as certain challenges, in some cases unique to the Latin American region.

This chapter presents a summary of instrumentation and computing related submissions to LASF4RI-HECAP. Both the state-of-the-art and challenges for the main technologies and projects are highlighted. Supporting instrumentation and computing research and development (R\&D) ecosystems is essential to attain the physics goals of the high energy physics, cosmology and astroparticle communities, as well as to build a relationship with industry, creating practical applications and transferring them to society.

The structure of this chapter is as follows. First, the main scientific key questions and non-scientific associated drivers are outlined. Second, common developments in the region are identified and described, emphasizing existing collaborations, their current status and technical challenges. Third, synergies and opportunities for are presented. Fourth, the topics of capacity building and capacity keeping are discussed. Finally, conclusions are presented.

\subsubsection{Main key scientific questions and highlights}

In the context of accelerator science and technology, the key scientific questions identified are the following:

- How to achieve proper complementarity for the high-intensity frontier vs the high-energy frontier?

- What are the options and challenges for accelerator technology?

For groups involved in collider experiments, successful completion of High-Luminosity Large Hadron Collider (HL-LHC) upgrade remains a priority (see also Sections 6.2). Institutions already participating in LHC experiments in Argentina, Brazil, Chile, Colombia, Cuba, Ecuador, Mexico and Peru expressed interest in continuing doing so in the HL-LHC concerning instrumentation and physics prospects studies. The situation is similar for groups in Brazil, Colombia, Paraguay and Peru participating in accelerator-based neutrino experiments. The groups closely follow the global/international discussions regarding future colliders: CLIC, ILC, HE-LHC, FCC-ee, FCC-hh and CEPC [I-2,I-3]. A detailed summary of the expected performance of the future colliders (in answer to the scientific drivers presented above) can be found in the Physics Briefing Book of the European Strategy for Particle Physics Update 2020 [1].

In the last few decades, the Latin American experimental community has grown considerably. There is a large diversity of activities ongoing in the region covering collider experiments, neutrino physics, nuclear physics, 
cosmology, astrophysics, astroparticles, gravitational waves and concrete capacity-building collaborations. The community's contributions to ambitious programs - like LHC experiments, the Pierre Auger Observatory, LAGO and GRID computing systems, among others - demonstrate its collective ability to engage in multinational and multidisciplinary endeavours effectively.

It is worth mentioning the existence of facilities in the region which could complement large scale projects for accelerators and experiments in particle, cosmology and astroparticle physics. For instance, the Brazilian Sirius Synchrotron Light Source and two particle accelerators dedicated to fundamental nuclear physics: one at the LAFN at the Institute of Physics of the University of Sao Paulo [I-4], and another called Tandar, at CNEA in Buenos Aires, Argentina. A strong collaboration with these technological facilities could be seminal for the realization of several scientific projects in the accelerator context in the region.

Regarding instrumentation, the scientific key questions identified are the following:

- What areas of instrumentation R\&D are ongoing in the region? And how do they meet the needs of future experimental programs?

- Are there possibilities of collaborations to boost the efforts?

Next-generation experiments include those at colliders, fixed-target and beam-dump facilities, as well as dedicated projects for the search of elusive particles. Some of the proposed programmes exploit synergies between particle physics, astroparticle physics and cosmology (i.e. CTA or SAGO). Depending on the physics goals and experimental conditions, the technological challenges are varied and include: improved spatial resolutions (few $\mu \mathrm{m}$ per hit, low mass) and time resolutions (down to $10 \mathrm{ps}$ per hit), high-performance photo-detectors, very high tolerance to radiation, extensive area coverage at low costs, a large number of channels, very high readout speed, enhanced performance for reconstruction and triggering, low threshold acquisition systems with low readout noise, increased exposure and/or single photon-electron resolution.

Regarding computing and software in high energy, cosmology and astroparticle physics, the landscape is shifting rapidly. New projects need to consider not only detectors and R\&D costs but also computing innovations, challenges and costs. Computing resources are nowadays becoming increasingly heterogeneous. Chip architectures and associated software and networking are under constant review and development for proper adaptation and optimizations.

Long-term computing projects are moving more and more towards open source and open data models, and hybrid combinations of public and private (academic + volunteer + commercial) resources are being explored. No less relevant, the data management and preservation processes, public repositories, and protocols need substantial redefinition for future experiments, whiles keeping in mind the budget limitations, as also expressed in the updated European Strategy for Particle Physics Update [1].

Taking those factors into account, the scientific key questions identified in the computing and software context are:

- How the scientific software and data structure must be designed to take the best advantage of the current resources in the different institutions in the region, and associated partners in other places of the world?

- How should computing evolve to support future scientific programs and their specific needs?

- How can current commercial solutions be a relevant player in more cost-effective usage of the funds allocated to computing power while keeping a pertinent role in the operations?

- What are the R\&D activities ongoing in the region? And how to boost them to enable the computing evolution while ensuring computation and science reproducibility as well as open access principles?

To try to address the identified scientific key questions, summaries of the technologies and their possible specific applications in the region (from the inputs received) are shown in sections 7.2 and 7.3 These summaries highlight the diversity of ongoing projects, as well as the need for coordinating bodies/panels in the region to maximize the scientific outcomes of these activities and make the most efficient use of resources. Such bodies/panels can oversee the creation of coherent strategies and collaboration in the region; opportunities for the development of, and access to common tools, infrastructures and services; development, deployment and access to an extensive network of information and worldwide expertise in different areas, wide dissemination of knowledge and results and a visible framework for institutions. 
The timescales spanned by ongoing and future projects, range from a few years to decades. There are short-term projects ( $<5$ years) like phase-I CMS, LHCb and ATLAS upgrades, ASTRID-mini array, AugerPrime and Reactor Neutrino experiments; and long-term ( $>5$ years) experiments like CTA, DUNE, HL-LHC and the American Gravitational Wave Observatory, and big facilities like ANDES. In addition to the complexity and diversity of the required technologies, these timescales also constitute a challenge. The challenge is related to having funding stability over a long period of time and creating regional programs that facilitate regional collaborations.

\subsubsection{Non-scientific drivers}

Non-scientific drivers are also important in this context. They include not only financial support, as stated before, but also:

- Access to shared infrastructures and tools. Considering the regional landscape is essential, i.e. einfrastructure and internet performance vary a lot in the region.

- The existence of effective collaborations and organizational networks, regional and international. It is important to build meaningful relations, and synergies with related strategy initiatives, like Snowmass ${ }^{1}$ and the European Particle Physics Strategy ${ }^{2}$

- The improvement of existing structures and the creation of new mechanisms through which the community can build relationships with the industry—also leveraging the career opportunities of those interdisciplinary associations in both directions.

- Environmental and social impact: knowledge transfer, outreach \& communication, and the ecological impact of our activities.

- Training of the next generation of experts while understanding the natural flow of human resources between the scientific and private sectors.

- Appropriate support and recognition of the workforce engaged in accelerators, instrumentation and computing R\&D activities.

The last two points are particularly crucial in the context of Latin America. To effectively carry out the projects submitted to LASF4RI and to increase the visibility of the teams in the region, we need: 1) more skilled physicists, engineers and computer scientists, and 2) significant investment. The last section will be devoted to addressing this. Finally, it is crucial to keep in mind that planning for the next years implies considering the impact of unpredictable events (like the current pandemic) on the timelines of the activities and the institutions involved.

\subsection{Topics within similar instrumentation drivers}

In this section common developments are identified in terms of dedicated devices and technologies that are being implemented as part of different detectors, observatories and experiments.

\subsubsection{FPGA Boards}

The recent developments in FPGA processor power and I/O bandwidths are enabling complex online feature extraction and increased readout flexibility. This also requires firmware development with increased complexity that demands higher level of expertise.

There is a general approach to use FPGAs boards, with projects in Argentina and Brazil, for a fast trigger decision and selection algorithms for the first level triggers, and back-end electronics of CMS [I-20] and ATLAS [I-2,I-19]. This approach is based on Calorimeter and Muon system information and includes efforts to implement challenging deep neural networks firmware algorithms. These projects include the development of new dedicated electronic laboratories in the region. The projects should be ready for the HL-LHC, which will start delivering collisions in 2026.

\footnotetext{
${ }^{1}$ Snowmass 2021: https://snowmass21.org

${ }^{2}$ European Particle Physics Strategy: https://europeanstrategy.cern
} 
FPGA Boards are the core of different general readout systems, which are included in the neutrino reactor instrumentation for the $v$-Angra [I-14] experiment in Brazil and in the new Skipper CCDs technologies [I-9,I15] for a Low Threshold Acquisition Systems for Dark Matter searches [1-22] to be developed in Argentina.

\subsubsection{Read Out systems}

In many projects that are proposed for the near future, which are being built now or are going through major upgrades, the readout systems are being implemented using new concepts for bridging the streams of data between various detector electronics and the endpoints of the data acquisition network. For the Run-3 upgrade of the ALICE experiment, groups in Brazil have greatly contributed with the full development of a new front-end readout chip (knwon as the SAMPA chip) for two ALICE detectors, the Time Projection Chamber (TPC) and the Muon Chamber $(\mathrm{MCH})$, and the construction of the mechanical support of the new Muon Forward Tracker (MFT) [I-10].

In the context of the ALICE Upgrade for LHC Run-4, the FoCal is a high-granularity calorimeter located in the very high rapidity region of the experiment, adding new capabilities. The planned contribution in this project is the R\&D and construction of the FoCal Silicon Pad layers readout system. Given the large experience of the group with the development of the SAMPA chip, which will instrument the ALICE TPC and MCH detectors beyond Run-3, it is natural to expect that a significant contribution to this part of the project can be achieved [I-10]. Brazilian participation in the ALICE experiment for the next 6 or 7 years includes projects for the LHC Run 3, from May 2021 to the end of 2024, and the preparation for the LHC Run 4 with upgraded components to be installed during the Long Shutdown 3, from 2025 to mid-2027.

Institutions in Colombia, collaborating with groups in Peru and Paraguay, are working directly on the design of the digitization boards of the DUNE experiment [I-1]. To read the SiPMs signals, digitization at room temperature will be performed by electronic boards, currently under design. The boards are known as DAPHNE (Detector Electronic for Acquiring Photons from Neutrinos) and will perform the digitization, initial processing and communication. Following this work at the beginning of the year 2020, the production of a small number of prototype boards is planned. The prototypes will be tested at different facilities around the world [1-7].

Different groups in Argentina have been involved in the development of the first and only existing electronics for the Skipper-CCD detectors [I-22]. The good performance has encouraged its use in more than 10 institutions in different countries. The newly developed readout system provides unique capabilities for low noise applications as required for the Skipper-CCD sensor.

\subsubsection{Small-area Photomultipliers (sPMTs)}

Small-area photomultipliers are designed to have a better single photon-electron resolution, with the ability to work in a "photon-counting mode".

The JUNO experiment consists of a set of 25000 of these photomultipliers in the so-called "counting mode". The Latin American activities, based in Brazil and Chile, are focused on the sPMT system with both hardware and software contributions [1-12]. The JUNO detector construction is well under way and test runs are expected to start in June 2022. Concerning the sPMT subsystem, 26000 sPMTs have already been tested and accepted well within schedule.

The Pierre Auger Observatory (PAO), located in Argentina, is going through a major Upgrade (AugerPrime). It includes the addition of a fourth SPMT in each Water Cherekov Detector (WCD), which will drastically reduce the number of events with saturated signals [1-17]. Brazilian groups will share efforts that involve the acquisition, characterization and assembly of the equipment in the PAO site in Argentina. They also foresee to operate these devices at least until the end of 2025.

\subsubsection{Silicon Photomultipliers (SiPMs)}

The Silicon Photomultiplier is a sensor that addresses the challenge of collecting, timing and quantifying lowlight signals down to the single-photon level. It combines low-light detection capabilities with the benefits of a solid-state sensor.

For DUNE and SBND experiments, groups in Brazil, Colombia and Paraguay [I-7,I-11] are collaborating on projects of cold electronics for the amplification of the SiPM and digitization of the signals with the DAQ 
system. To increase the effective collection area of SiPMs, the ARAPUCA concept has been developed in Brazil, based on the shifting and trapping of scintillation light in noble liquids.

The ASTRI MINI-ARRAY telescopes are characterized by an optical system based on a dual-mirror SchwarzschildCouder design and a camera at the focal plane composed of silicon photomultiplier sensors managed by fast read-out electronics of custom design [I-5, I-6, I-24]. The ASTRI MINI-ARRAY is the precursor of CTA to be installed in Tenerife and with start-up date set to 2022. Brazilian engineers have been involved in the development of the prototype as well as in the construction of the camera. They will participate in the final testing of the telescope and foresee future manufacturing in Brazil of other telescopes for the CTA-South Array. The first telescopes are expected to be installed on site in 2020 in the north and in 2022 in the south. The completion of the first phase of operations, with a subset of telescopes, and the start of a second phase, for the installation of the full array, is planned for the beginning of 2025.

\subsubsection{Charge-Coupled Devices (CCDs and Skipper CCDs)}

CCDs and the new Skipper version are semiconductor device sensors that can achieve deep sub-electron readout noise levels and single electron counting capabilities.

There is a proposal to upgrade the CONNIE experiment with Skipper CCDs, which will allow it to achieve lower detection thresholds and greatly increase its sensitivity [I-9]. The plan is to install these new devices in 2020 in order to study their performance as low-energy neutrino coherent scattering detectors, as well as to characterise the backgrounds and perform noise and stability studies. Based on the results of this pilot program, further plans will be done on how to size up the detector.

There is a wider effort in Latin America to build the next big reactor neutrino experiment using Skipper CCDs [1-15]. One strong contender for the experiment site is the Atucha reactor in Argentina, which may allow placing the detector at a minimal distance of $12 \mathrm{~m}$ from the reactor core, inside its dome, thus increasing the neutrino flux and also profiting from the concrete shielding from the dome to decrease the cosmic muon background [I-9].

Efforts are ongoing to develop a new Smart Skipper-CCD camera to install it in new instruments [I-22]. This proposal seeks to develop technology and scientific forecasts for next-generation cosmic surveys probing the 'dark sector'. Specific aims are: (1) to develop faster readout strategies for low-noise Skipper CCDs, (2) to characterize the optical performance of these detectors for their use in quantum imaging applications and

cosmic surveys, (3) to demonstrate the first implementation of Skipper CCDs on a prototype astronomical instrument, and (4) to assess the sensitivity of future cosmic surveys to fundamental properties of dark matter. A 5-year plan has been developed to achieve these goals.

It is also worth mentioning that the TOROS experiment aims to perform optical follow-ups of gravitational-wave events conducted by unfiltered CCD observations.

\subsubsection{Resistive Plate Chambers (RPC)}

Resistive Plate Chambers are gaseous parallel-plate detectors that combine precise spatial resolution with a good time resolution. They are, therefore, well suited for fast space-time particle tracking, in particular for muons.

As part of the PAO upgrade that is currently ongoing, it is expected that the RPC assembled in Brazil [I-17] will be tested and operated on-site in the coming years.

The Muon Upgrade project of CMS is an international effort with important contributions from institutions in Brazil, Colombia and Mexico. The upgrade includes the installation of a new Link System for the RPC system and new chambers in the high eta regions [I-1, I-20]. This project will be finished with the installation of the new RPC chambers and electronics during the Yearly Technical Stops at the end of 2022 and 2023. The collaboration, with participation of institutions in Brazil, proposes also updating the readout software for Run-3, and the data certification using Machine Learning $(M L)$ techniques to evaluate performance. 


\subsubsection{ARAPUCA Light Trap (Argon R\&D Advanced Program at UNICAMP)}

Liquid Argon Time Projection Chambers are a choice for the next generation of large neutrino detectors due to their optimal performance in particle tracking and calorimetry. ARAPUCA offers a light trap device to enhance Ar scintillation light collection (and thus the overall performance) based on a suitable combination of dichroic filters and wavelength shifters.

The far detector of the DUNE experiment will consist of a liquid Argon Time Projection Chamber (TPC). Dimmer light, coming from the most remote regions of the TPC, needs to be collected. For that reason, a large photon collection is required at the far end, made possible through the use of a new technology developed in Brazil [I-7,I-13]: the ARAPUCA concept.

The next-generation X-ARAPUCA concept currently represents the baseline choice for the photon detection system of the DUNE experiment. The X-ARAPUCA is a development of the traditional ARAPUCA. This concept was conceived to reduce losses on the internal surfaces of the ARAPUCA by diminishing the average number of reflections before detection [I-13]. These projects will follow the DUNE timeline and aim to be ready for operations starting in 2026, while planning for 10 years data taking in order to achieve ambitious physics results.

\subsubsection{Water Cherenkov Detectors}

Water Cherenkov Detectors are based on a very well established technique in which Cherenkov radiation, generated by charged particles going through water, is detected using photomultiplier tubes. The technique provides the possibility to cover huge areas of target mass at reasonable costs. Many countries are working on the development, construction and operation of Water Cherenkov detectors.

At present, for the PAO and for different sites for the LAGO project [I-1,I-17], work is ongoing to incorporate new developments of electronic readout, DAQ custom systems, and the modelling and simulation of signals.

To be constructed in the Southern Hemisphere, the Southern Wide-field-of-view Gamma-ray Observatory (SWGO) is a next-generation WCD instrument with sensitivity to the very-high-energy band [I-23]. It will provide a unique view on gamma-ray and cosmic-ray emission from tens of GeV to hundreds of TeV. The facility will improve upon the success of the HAWC Gamma-ray Observatory in Mexico, which is surveying the northern gamma-ray sky. The recently formed SWGO Collaboration will conduct site selection and detector optimization studies prior to construction, with full operations foreseen to begin in 2026.

The Neutrino Angra Experiment is a water-based Cherenkov detector located and oparational in the Angra dos Reis nuclear power plant [I-14]. In addition to the detector itself, a complete data acquisition system has been designed and integrated for the experiment. The long term goal of the experiment is to develop a reliable and cost-effective technology to routinely monitor the nuclear reactor power and possibly the neutrino spectral evolution.

\subsubsection{Laser Interferometer}

Laser interferometers detect gravitational waves that extend and contract the distance between mirrors.

After 2030, two new gravitational wave observatories will likely step in: the Einstein Telescope and the Cosmic Explorer. Both will be based on Laser Interferometer technology. They will be the first ones of the so-called third-generation (3G). They will need a third partner for triangulation of arrival times to determine more precisely the source position in the sky. Therefore, there will be an opportunity for the construction of a South American Gravitational wave Observatory (SAGO), if the Latin American community reaches, by that time, a critical mass of experimentalists already educated in the $3 \mathrm{G}$ technology.

The main goal is the construction and the subsequent operation of a $3 \mathrm{G}$ laser interferometer for gravitational wave observation in South America [16]. Currently, only two group in Latin America are involved in the LIGO Scientific Collaboration and one in Virgo. All three groups are in Brazil. And there are only a few Latin Americans involved in laser interferometer projects. Therefore, the challenges to overcome in order to propose a project for the construction and development of a gravitational wave observatory in South America are enormous. 


\subsection{Computing and software}

Worldwide, the computing requirements of large experiments have been in a state of constant evolution. Innovative and flexible computing solutions and services have been developed to meet the numerous challenges of multiple experiments, use cases and scientific disciplines.

Regarding that evolution, several elements stand out: the hardware architectures and dispositions, the complexity and sizes of the datasets produced and the distributed nature of the researchers, engineers and students that form the human resource in those experiments.

\subsubsection{General remarks}

On the side of hardware - computer processing power, networks, storage elements and so on - one successful approach is the integration of already existing infrastructure to develop and consolidate linked macro-structures that perform as a single entity. Initiatives like the Worldwide LHC Computing Grid (WLCG) ${ }^{3}$ demonstrates how a very heterogeneous set of computer centres, universities and dedicated High-Performance Computing (HPC) facilities can join resources (including human expertise) to consolidate a global network. Such a system is currently serving multiple LHC and other experiments. More recently it was even used to collaborate in the relevant initiative far away from the HEP, like Folding@home 4 which is a distributed computing project to carry out complex simulations of protein molecules, how they fold, and how their movements are implicated in a variety of diseases.

On the software side, constant changes and bigger collaborations have emerged inside the global scientific community. The sophistication needed to carry out the data acquisition, data handling and, of course, the data analysis of those datasets collected (and simulated) by large experiments forces us to develop a more global approach, keeping close contact with the appropriate enterprises, protocols and industrial standards. In this context, scientific software and computational tools have earned a place by themselves as fields of study and careers to pursue.

The often-heard statement: "bad software is extremely expensive", refers to the fact that researchers have been encouraged to enhance their computing and software skills so that their analysis tasks take good advantage of the new and legacy hardware. Also, by using leading programming languages and techniques available, researchers can optimize running time and CPU usage. The lack of such enhancements and optimizations could have a negative impact on the overall performance of the system and consequently on the ratio of results/resources or, on a more pragmatic view: result/budget.

In the Latin American region, synergies and collaborations have emerged thanks to the participation of multiple institutions in multinational experiments and organisations. This is intimately related to the capacity building that we will address in the next section.

Nowadays, ongoing scientific ventures and any new project need to consider the computational aspects as part of their R\&D initiatives.

\subsubsection{Large collaboration examples in the region}

A couple of concrete efforts shown at the LASF4RI-HECAP workshop come from Brazil:

- At the CONNIE experiment, data is currently stored, processed and analysed in the CHE cluster at CBPF, which is the only machine allowed by Electronuclear to connect directly to the laboratory hosting CONNIE at Angra 2. The cluster has a professional storage system with currently 100TB dedicated to CONNIE and 280 cores available for CONNIE processing [I-9].

- In ALICE: several groups have been providing its fair share contribution to the experiment since 2007 through the SAMPA cluster (acronym from the Portuguese, Sistema de Analise e Multi-Processamento Avancado), hosted in the Physics Institute of Universidade de São Paulo. Currently, the cluster has 2408 CPU cores, totaling $18.7 \mathrm{kHS} 06$ of processing power and $0.85 \mathrm{~PB}$ of storage. It is important to highlight that part of this processing capability, around six kHS06, benefits local users, outside the GRID [I-10].

\footnotetext{
${ }^{3}$ Worldwide LHC Computing Grid (WLCG): https://wlcg.web.cern.ch/

${ }^{4}$ Folding@home: https://foldingathome.org
} 


\subsubsection{Training and knowledge transfer efforts}

More events, schools, workshops and trainee ships in the experiments looking to consolidate the flow of knowledge and expertise between students, scientists and industries are needed. They should address how to develop and deliver the right software infrastructure and tools, as well as how to effectively integrate experts and new members to the development of such common tools and protocols. An emblematic case is the HEP Software Foundation (HSF) ${ }^{5}$ that looks to facilitate cooperation and common efforts in High Energy Physics software and computing internationally.

Two relevant examples in the Latin American region that took place in 2019 are: the Latin American Workshop on Software and Computing (S\&C) Challenges in High-Energy Particle Physics (LAWSCHEP) ${ }^{6}$ and the Symposium Artificial Intelligence for Science, Industry and Society (AISIS) 7 in Mexico. LAWSCHEP -under the auspices of the HSF and with support from the Institute for Research \& Innovation in Software for HEP (IRIS-HEP)- brought together the Latin American HEP groups to discuss opportunities for collaboration in topics of S\&C within the region and with the international community at large. In addition, AISIS looked to bringing together and creating synergies between different scientific research and industries using new $S \& C$ technologies, including ML and other Artificial Intelligence (AI) branches, in fundamental research, ecology and also social disciplines.

In general, the submitted proposals show that we have already multiple international collaborations working in the development of cutting-edge hardware, computing networks and sophisticated software. They also show the need to encourage communication between Latin American countries to strengthen collaborations in current and new projects. The next step is to wrap-up those efforts with a constant and coordinated flow of high-level training. This is essential in order to reach these goals of long-term scientific production and qualified human capital, which can later on integrate other scientific and economic activities in the region.

\subsection{Synergies with other chapters/scientific topics}

Instrumentation and computing development for high energy, cosmology and astroparticle physics is both a driver for and a beneficiary of progress made within the topical sections presented before in this document. Some examples are: the development of silicon photomultiplier for neutrino experiments, pure water Cherenkov with photomultipliers and CCDs and Skipper CCDs.

A diversity of other areas benefit from and synergistically contribute to the advances in accelerators, instrumentation and computing. Some of the most significant examples mentioned in the inputs received are:

- Computer science: collaboration in the use of cloud computing, big data and external storage services. Usage and development of Machine Learning/Artificial intelligence [I-1, I-2, I-21].

- Sharing of "generic" resources and trainings, including computing, software development and management, consultancy with external parties, like companies, is important for the computing and software development side [I-21].

- The UniANDES group in Colombia participates in interdisciplinary applications of semiconductor and gaseous radiation detectors [I-1].

- Geophysics: the work done by the UIS group in Colombia on cosmic ray detection has led to other projects such as the Muon Telescope (MuTe) for muon volcanography [I-1].

- Citizen science: the Red Ambiental Cludadana de MOnitoreo (RACIMO) initiative is a citizen network for environmental monitoring from the UIS group in Colombia [I-1].

- Space weather: Paraguayan groups started working with space weather due to its location at the center of the South Atlantic Magnetic Anomaly. They are involved in the installation of magnetometers in order to be part of the EMBRACE net, which measures the magnetic field in South America and of a new muon detector that will help measuring not only the flux, but the incoming direction as well [l-11].

- Nuclear physics: Argentina and its series of dedicated reactors and the collaboration between them due to a single overseeing agency.

\footnotetext{
${ }^{5}$ HEP Software Foundation (HSF): https://hepsoftwarefoundation.org

${ }^{6}$ Latin American Workshop on Software and Computing (S\&C) Challenges in High-Energy Particle Physics (LAWSCHEP): https://indico.cern.ch/event/813325/

${ }^{\top}$ Symposium Artificial Intelligence for Science, Industry and Society (AISIS): https://indico.cern.ch/event/781223/
} 
- Other known synergies were not specifically addressed in the inputs received but they are worth mentioning: medical physics, biology, photonics and neutronics, large civil, vacuum and cryogenic infrastructures, among others.

An important aspect of this exercise is to identify these synergies and support/encourage them through the funding of interdisciplinary research. The joint developments with applied fields in academia and industry are also essential for advances in accelerators, instrumentation and computing.

\subsection{Developing and preserving knowledge and expertise}

Worldwide, one of the challenges faced by the particle physics and cosmology communities is to ensure an adequate level of development and preservation of expertise in instrumentation and computing-related R\&D activities. In Latin America, the situation is not different and it is deepened by episodic funding, and subcritical mass in instrumentation- and computing-trained human resources in the individual institutions.

Some efforts to develop and retain expertise in the region are ongoing. At the training level, enhancing fundamental physics, instrumentation and computing training at the university level by multiple members of the Latin American community (e.g. CEVALE2VE $[2]^{8}$, LA-CoNGA physics $[1-8]^{9}$ and investing in specialized schools (e.g. CERN-Latinamerican schoo ${ }^{10}$ ) is highly beneficial.

However, available economic and human resources mean that universities are typically limited. Hence, creating regional training programs with clusters of institutions participating is an appealing option (e.g. LA-CoNGA physics [I-8], PPGCosmo experience [I-31]). Pursuing these ideas will need investment in TechEd and digital education skills in the region. It will be a great opportunity to invite other countries in the region not currently actively working in the field (and not in the LASF4RI-HECAP network yet) to include such topics in their university programs.

Regarding expertise preservation and career opportunities, even the visibility given by academia often cannot compensate for the higher salaries and attractive challenges in industry for physicists and engineers with instrumentation and/or computing skills. Ways for academic institutions to provide stable employment and more competitive wages for these crucial professionals must be found with specialized and attractive grants, as well as career prizes for instrumentation and computing R\&D. Exploiting synergies with engineering and computing science departments is also important (as stated in several of the inputs received). Efforts in outreach and communication are welcome, not only to attract more students but also to increase the positive view at the society and governmental level of particle physics and cosmology like a playground for innovation with far-reaching impact on industry and society.

\subsection{Conclusions}

The presence and construction of new local facilities and infrastructures to develop, build, test and characterise new instruments, technologies and detectors are growing significantly in the region. The review presented in this chapter is based on the different inputs that were submitted to LASF4RI and show the large diversity of experience and skills developed in the different groups and institutions. They already show a large number of projects and involvement in international collaborations. Many of them are now at the point where collaborative efforts, knowledge sharing and facilities will be boosting the local efforts towards significant contributions to sciences with great international implications.

It is crucial for the community to maintain a strong focus on instrumentation and computing R\&D and to foster an environment that stimulates innovation. Creating this environment depends on several factors, including: stable funding, access to shared infrastructures and tools, existence of networks and consortia and the training, recognition and retention of human resources.

Capacity building must be a priority in the region. It is important to highlight that those high-level knowledge transfer and educational efforts must take into account the local situations, and also aim to generate a critical mass of human resources that remains in the region so that a long-term and progressive development of such projects can be a sustained reality.

\footnotetext{
${ }^{8}$ CEVALE2VE: http://www.cevale2ve.org

${ }^{9}$ Latin American Alliance for Capacity buildiNG in Advanced Physics (LA-CoNGA physics): https://laconga.redclara.net

${ }^{10}$ CERN-Latinamerican school: https://www.symmetrymagazine.org/article/building-the-future-two-weeks-at-a-time
} 
Finally, LASF4RI-HECAP is the first overall regional effort to bring together the Latin American particle, astroparticle physics and cosmology community. It offers an invaluable opportunity to perform surveys and create working panels to assess the global status of the community in terms of demographics, resources, early-career scientists status, career recognition and perspectives, and diversity, among others. 


\section{Bibliography}

[1] Richard Keith et al. Ellis. Physics Briefing Book: Input for the European Strategy for Particle Physics Update 2020. Technical Report arXiv:1910.11775, Geneva, Oct 2019. 254 p.

[2] Arturo Sánchez Pineda. The CEVALE2VE case. Technical Report ATL-OREACH-PROC-2017-001, CERN, Geneva, Jan 2017. 


\section{Appendix}

\subsection{List of White Papers}

Table 8.1: Details of the Inputs of White Papers for this Section

\begin{tabular}{|c|c|}
\hline Input IL & Title \\
\hline I-1 & Colombian Network on High Energy Physics - Input on Experimental HEP \\
\hline $1-2$ & Argentina Experimental HEP Input \\
\hline 1-3 & Brazilian Participation in the Next-Generation Collider Experiments (Young Scientists) \\
\hline 1-4 & White Paper on Nuclear Science in Brazil. Contribution to the Latin American Strategy Forum for Research Infrastructure \\
\hline $1-5$ & The ASTRI MINI-ARRAY: a Precursor for the Cherenkov Telescope Array (CTA) \\
\hline $1-6$ & The Cherenkov Telescope Array (CTA) \\
\hline 1-7 & DUNE in the context of LASF4RI. The Colombian Case \\
\hline $1-8$ & LA-CoNGA Physics perspectives for the Latin America Strategy Forum for Research Infrastructure \\
\hline I-9 & Coherent Neutrino-Nucleus Scattering Experiment (CONNIE) \\
\hline $1-10$ & The Study of the Quark-Gluon Plasma with the ALICE-LHC Experiment \\
\hline 1-11 & Letter of Intent of the Paraguayan Group \\
\hline $1-12$ & Latin American Contribution to JUNO \\
\hline $1-13$ & Brazilian Community Report on Neutrino Physics \\
\hline $1-14$ & Brazilian Report on Safeguards Application of Reactor Neutrinos \\
\hline $1-15$ & Short baseline neutrino experiment in nuclear reactors in Argentina \\
\hline $1-16$ & The South American Gravitational wave Observatory (SAGO) White Paper \\
\hline 1-17 & Unravelling the Mysteries of Ultraenergetic Cosmic Rays with AugerPrime \\
\hline $1-18$ & Physics exploration with the LHCb experiment. LHCb Group in Brazil \\
\hline $1-19$ & The ATLAS/Brazil Cluster: Current Status and Perspectives from the ATLAS Upgrade Programme \\
\hline $1-20$ & CMS Group - Universidade do Estado do Rio de Janeiro (UERJ)- This contribution was retracted. \\
\hline $1-21$ & A proposal for Transversal Computer-related Strategies and Services for Scientific and Training efforts \\
\hline $1-22$ & Developing the first astronomical and quantum imaging instrument using the Smart Skipper-CCD technique \\
\hline 1-23 & Southern Wide-field-of-view Gamma-ray Observatory (SWGO) \\
\hline 1-24 & The Cherenkov Telescopes Array: fundamental physics and instrumentation \\
\hline $1-25$ & GRAND: Giant Radio Array for Neutrino Detection \\
\hline $1-26$ & A Venezuelan input to the Latin American Strategy for Research Infrastructures (LASF4RI) \\
\hline 1-27 & Colombian Network on High Energy Physics Input on Theoretical HEP \\
\hline $1-28$ & Hyper-Kamiokande: Possible Contributions from Latin America \\
\hline I-29 & The Latin American Giant Observatory \\
\hline $1-30$ & Ecuadorian HECAP Groups Input to the Latin American Strategy Forum for Research Infrastructure \\
\hline 1-31 & A Latin American graduate school - The PPGCosmo experience \\
\hline $1-32$ & The ANDES Deep Underground Laboratory \\
\hline $1-33$ & Macón Ridge Astronomical Site \\
\hline 1-34 & Brazilian Community Report on Dark Matter \\
\hline $1-35$ & Dark Matter and Neutrino Physics - Contribution from Buenos Aires to the LASF4RI \\
\hline $1-36$ & An Andean Deep-Valley Detector for High-Energy Tau Neutrinos \\
\hline $1-37$ & QUBIC: Q\&U Bolometric Interferometer for Cosmology \\
\hline $1-38$ & Cosmology with the Large Synoptic Survey Telescope (LSST) \\
\hline $1-39$ & e BINGO Radio Telescope: an instrument to investigate the Universe through the $21 \mathrm{~cm}$ neutral hyc \\
\hline
\end{tabular}




\subsection{Glossary of Experiments}

ABRAS: Argentina-Brasil Astronomical Center

ALICE: A Large lon Collider Experiment

ALPACA: Andes Large-area PArticle detector for Cosmic-ray physics and Astronomy

ANDES: Agua Negra Deep Experiment Site

ANGRA: Angra Dos Reis Nuclear complex

ASTRI: Astrofisica a Specchi con Tecnologia Replicante Italiana

ATLAS: A Toroidal LHC ApparatuS

AUGER: Pierre Auger Observatory

BELLE 2: B detector at KEK

BINGO: BAO from Integrated Neutral Gas Observations

CEPC: Circular Electron-Positron Collider

CLAS: CEBAF Large Acceptance Spectrometer

CMS: Compact Muon Solenoid

CNEA TANDAR: Acelerador TANDAR, Comisión Nacional de Energía Atómica

CONNIE: Coherent Neutrino Nucleus Interaction Experiment

CTA: Cherenkov Telescope Array

DARKSIDE: Two phase TPC for Dark Matter Direct Detection

DUNE: Deep Underground Neutrino Experiment

FCC: Future Circular Collider

GRAND: Giant Radio Array for Neutrino Detection

HAWC: High Altitude Water Cherenkov Gamma-ray Observatory

HL-LHC: High Luminosity Large Hadron Collider

Hyper-K: Hyper Kamiokande

ILC: International Linear Collider

JUNO: Jiangmen Underground Neutrino Observatory

KM3NeT: Cubic Kilometre Neutrino Telescope

LAGO: Latin American Giant Observatory

LAFN Tandem: Tandem Accelerator at Laboratorio Aberto de Fisica Nuclear

LHCb:Large Hadron Collider beauty

LLAMA: Large Latin American Millimetre Array

LSST: Legacy Survey of Space and Time

NovA: NuMI Off-axis ve Appearance

PHENIX:Pioneering High-Energy Nuclear Interaction eXperiment

PPS: Precision Proton Spectrometer

QUBIC: Q\&U Bolometric Interferometer for Cosmology

RHIC: Relativistic Heavy lon Collider

SAGO: South American Gravitation Wave Observatory

SBND: Short Baseline Neutrino Detector

SENSEI: Sub-Electron-Noise Skipper Experimental Instrument

STAR:Solenoidal Tracker at RHIC

SWGO:Southern Wide-field-of-view Gamma-ray Observatory

TAMBO:Tau Air Shower Mountain-Based Observatory

TOROS: The Transient Optical Robotic Observatory of the South

$v$ IOLETA:Neutrino Interaction Observation with a Low Energy Threshold Array 